\title{
Mineralogy from Cores in Prospect Gulch, San Juan County, Colorado
}

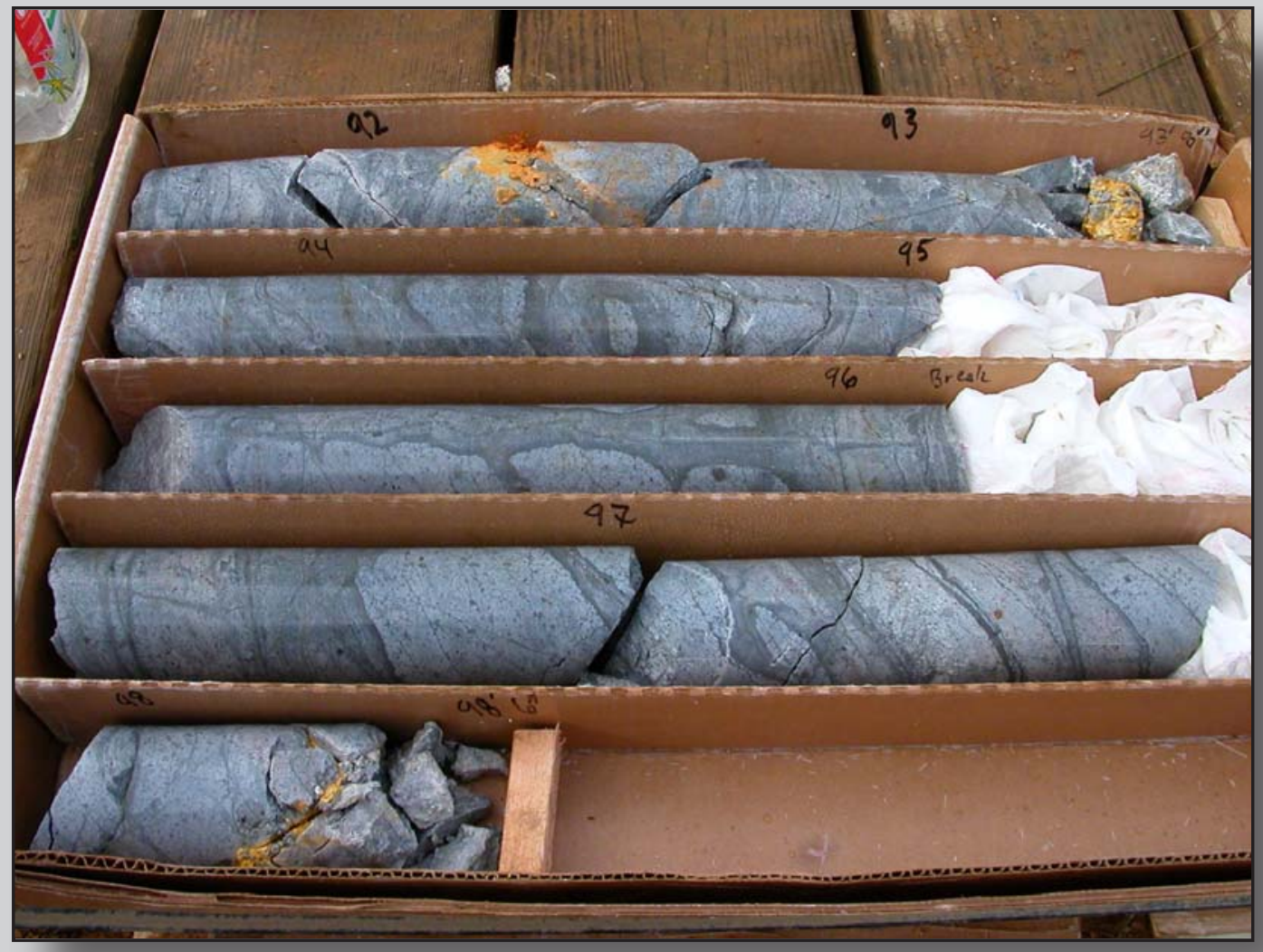

Open-File Report 2007-1095 


\section{Mineralogy from Cores in Prospect Gulch, San Juan County, Colorado}

By Dana J. Bove, Raymond H. Johnson, and Douglas B. Yager

Open-File Report 2007-1095 


\title{
U.S. Department of the Interior DIRK KEMPTHORNE, Secretary
}

\section{U.S. Geological Survey \\ Mark D. Myers, Director}

\section{U.S. Geological Survey, Reston, Virginia: 2007}

For product and ordering information:

World Wide Web: http://www.usgs.gov/pubprod

Telephone: 1-888-ASK-USGS

For more information on the USGS - the Federal source for science about the Earth its natural and living resources, natural hazards, and the environment:

World Wide Web: http://www.usgs.gov

Telephone: 1-888-ASK-USGS

\author{
Any use of trade, product, or firm names is for descriptive purposes only and does not imply \\ endorsement by the U.S. Government. \\ This publication has not been reviewed for stratigraphic nomenclature \\ Although this report is in the public domain, permission must be secured from the individual \\ copyright owners to reproduce any copyrighted material contained within this report. \\ Suggested citation: \\ Bove, D.J., Johnson, R.H., Yager, D.B., Mineralogy from Cores in Prospect Gulch, San Juan County, Colorado: U.S. \\ Geological Survey, Open-File Report 2007-1095, 82 p.
}




\section{Contents}

Abstract
Introduction.
Geologic Setting
Hydrologic Setting
Drilling Locations, Core Logs, and Oxidation Depth
Mineralogy from Cores
References Cited
Appendix A. Core log for UPG-D
Appendix B. Core log for MPG-D
Appendix C. Core log for LPG-D

\section{Figures}

1. Location of Prospect Gulch in the upper Animas River watershed ..................................5

2. Detailed alteration map of bedrock in Prospect Gulch and the surrounding area............6

3. Drill hole locations in Prospect Gulch.........................................................................

4. Depth of the partially oxidized zone in feet ...................................................................

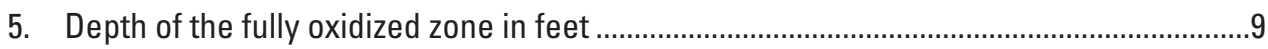

\section{Tables}

1. Drill hole locations and oxidation depths ……….........................................................10

2. Mineralogy in UPG-D samples from $X$-ray diffraction analyses.....................................11

3. Mineralogy in MPG-D samples from $X$-ray diffraction analyses....................................14

4. Mineralogy in LPG-D samples from $X$-ray diffraction analyses....................................20

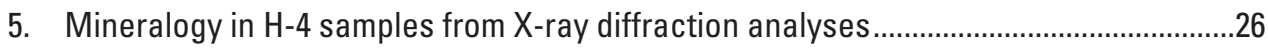

6. Mineralogy in $\mathrm{H}-5$ samples from $\mathrm{X}$-ray diffraction analyses.........................................32

7. Mineralogy in $\mathrm{H}-6$ samples from $\mathrm{X}$-ray diffraction analyses.............................................34 


\section{Conversion Factors}

Inch/Pound to SI

\begin{tabular}{lcl}
\hline \multicolumn{1}{c}{ Multiply } & By & \multicolumn{1}{c}{ To obtain } \\
\hline & Length & \\
\hline inch (in.) & 2.54 & centimeter $(\mathrm{cm})$ \\
inch (in.) & 25.4 & millimeter $(\mathrm{mm})$ \\
foot (ft) & 0.3048 & meter $(\mathrm{m})$ \\
mile (mi) & 1.609 & kilometer $(\mathrm{km})$ \\
yard (yd) & 0.9144 & meter $(\mathrm{m})$ \\
\hline
\end{tabular}

Temperature in degrees Fahrenheit $\left({ }^{\circ} \mathrm{F}\right)$ may be converted to degrees Celsius $\left({ }^{\circ} \mathrm{C}\right)$ as follows:

$$
{ }^{\circ} \mathrm{C}=\left({ }^{\circ} \mathrm{F}-32\right) / 1.8
$$

Vertical coordinate information is referenced to the North American Vertical Datum of 1929 (NAVD 29).

Horizontal coordinate information is referenced to the North American Datum of 1927 (NAD 27).

SI to Inch/Pound

\begin{tabular}{lll}
\hline \multicolumn{1}{c}{ Multiply } & By & \multicolumn{1}{c}{ To obtain } \\
\hline & Length & \\
\hline centimeter $(\mathrm{cm})$ & 0.3937 & inch (in.) \\
millimeter $(\mathrm{mm})$ & 0.03937 & inch (in.) \\
meter $(\mathrm{m})$ & 3.281 & foot (ft) \\
kilometer $(\mathrm{km})$ & 0.6214 & mile (mi) \\
meter $(\mathrm{m})$ & 1.094 & yard (yd) \\
\hline
\end{tabular}

Temperature in degrees Celsius $\left({ }^{\circ} \mathrm{C}\right)$ may be converted to degrees Fahrenheit $\left({ }^{\circ} \mathrm{F}\right)$ as follows:

$$
{ }^{\circ} \mathrm{F}=\left(1.8 x^{\circ} \mathrm{C}\right)+32
$$

Vertical coordinate information is referenced to the North American Vertical Datum of 1929 (NAVD 29).

Horizontal coordinate information is referenced to the North American Datum of 1927 (NAD 27). 


\title{
Mineralogy from Cores in Prospect Gulch, San Juan County, Colorado
}

\author{
By Dana J. Bove, Raymond H. Johnson, and Douglas B. Yager
}

\section{Abstract}

In the late nineteenth century, San Juan County, Colorado, was the center of a metal mining boom in the San Juan Mountains. Although most mining activity ceased by the 1990s, the effects of historical mining continue to contribute metals to ground water and surface water. Previous research by the U.S. Geological Survey identified ground-water discharge as a significant pathway for the loading of metals to surface water from both acid-mine drainage and acid-rock drainage. In an effort to understand the ground-water flow system in the upper Animas River watershed, Prospect Gulch was selected for further study because of the amount of previous data provided in and around that particular watershed. In support of this ground-water research effort, data was collected from drill core, which included: (1) detailed descriptions of the subsurface geology and hydrothermal alteration patterns, (2) depth of sulfide oxidation, and (3) quantitative mineralogy.

\section{Introduction}

In the late $19^{\text {th }}$, San Juan County, Colorado, was the center of a metal mining boom in the San Juan Mountains. Although most mining activity ceased by the 1990s, the effects of historical mining continue to contribute metals to ground water and surface water. These increased metal concentrations degrade stream-water quality and are toxic to fish and other aquatic organisms. As a result, viable fish and aquatic habitat is now more limited than what existed before mining (Besser and others, 2006). Since the 1990s, the local economic base has shifted away from hard-rock mining toward tourism with a focus on recreational opportunities. This new focus, as well as increased population downstream also has increased the demand for clean water. While streams in this area have low $\mathrm{pH}$ and elevated metal loads due to acid-rock drainage, the influence of acid-mine drainage due to historical mining activities has degraded preexisting ground-water and surface-water quality (Church and others, 2006). Determining the ground-water flow and associated dissolved-metal transport is critical to protecting ground-water and surface-water resources, determining the influence of acid-mine drainage compared to preexisting acid-rock drainage, and providing sensible remedial options.

Much of the San Juan Mountains is influenced by historical mining, and many surface-water samples (along with other data) have been collected in the upper Animas River watershed (fig. 1) by the U.S. Geological Survey (Church and others, 2006; http://amli.usgs.gov/reports/). Three goals of this watershed characterization project were to (1) characterize the surface-water quality, (2) identify abandoned mines that contribute the greatest metal loads to surrounding surface waters, and (3) determine premining water quality. The ultimate objective was to provide the necessary scientific information for public land managers to select effective remedial approaches that would improve watershed quality. Ground-water discharge has been identified as a significant pathway for the loading of metals to surface water from both acid-mine drainage and from acid-rock drainage (Church and others, 2006; Mast and others, 2006; Kimball and others, 2002; Kimball and others, 2006). Understanding the ground-water flow and dissolved metal transport is essential in determining whether sampled metal loads to streams are related to acid-mine drainage or acid-rock drainage, and thus, whether or not an identified source of metal loading should be remediated.

In an effort to understand the ground-water flow system in the upper Animas River watershed, Prospect Gulch (fig. 1) was selected for further study because of the amount of data provided in and around that particular watershed (Church and others, 2006), including stream tracer studies (Kimball and others, 2002; Wirt and others, 1999, 2001) and detailed maps of hydrothermal alteration (Bove and others, 2006). In addition, many of the inactive mines within Prospect Gulch are on land managed by the Bureau of Land Management (BLM). An understanding of the ground-water flow system is critical in assisting them with their remedial efforts. In support of this ground-water research effort, data was collected from drill core, which included: (1) detailed descriptions of the subsurface geology and hydrothermal alteration patterns, (2) depth of sulfide oxidation, and (3) quantitative mineralogy. The data from these cores will be used to support the calibration of a ground-water model in this area and provide the basis for a holistic model of the hydrogeochemistry of Prospect Gulch. This report summarizes all of the core related mineralogy that was collected. 


\section{Geologic Setting}

The predominant rock types in the Prospect Gulch study area consist of intermediate- to silicic-composition igneous rocks. These rocks formed following formation of the 28.2-Ma San Juan caldera, coincident with and (or) postdating formation of the 27.8-Ma Silverton caldera (Yager and Bove, 2002). Intermediate-composition lava flows and minor volcaniclastic sedimentary rocks that were deposited on the flanks of central vent volcanoes predominate this igneous sequence and are part of the Silverton Volcanics described by Lipman and others (1973). Silverton Volcanics lavas and volcaniclastic sediments shed from the adjacent volcanoes infilled the San Juan caldera volcanic depression to nearly a kilometer in thickness over an approximately 14-km-diameter area. Primary igneous minerals of the Silverton Volcanics intermediate-composition porphyritic lavas include, in relative order of abundance, plagioclase, quartz, hornblende, pyroxene, \pm biotite, and opaque oxide minerals.

A regional propylitic alteration event affected much of the study area following caldera formation (Burbank, 1960). The propylitic alteration formed as the large thickness of lavas that infilled the San Juan caldera cooled and degassed, altering the primary igneous mineral assemblage to a secondary assemblage containing chlorite, \pm epidote, \pm calcite, \pm pyrite, fine-grained muscovite, and iron oxide minerals. This propylitic assemblage was shown to have some acid-neutralizing capacity where it was not more intensely altered by later hydrothermal alteration events (Yager and others, 2005).

Prospect Gulch is located on the margin of the historic Red Mountain mining district, where ore deposits of silver, copper, lead, and zinc sulfides were discovered in 1881 (Burbank, 1947) and is located near the northwest structural margins of the San Juan and Silverton calderas. This area is the locus for several small-volume plugs and dikes of dacite to rhyolite composition that intruded along the structural margins of the calderas, after caldera activity ceased. Isotopic ages of intrusions near Prospect Gulch indicate that they also formed several million years after eruption of the intermediatecomposition Silverton Volcanics lavas (unit Tsv as noted in Appendix core logs) during the Miocene Epoch, which are thought to have provided the heat source for the hydrothermal system that was responsible for mineralization and associated alteration in and adjacent to the Red Mountain mining district (Lipman and others, 1976; Bove and others, 2001).

Several alteration types formed contemporaneously with or shortly after intrusive activity at approximately $23 \mathrm{Ma}$ (Bove and others, 2001), overprinting the regional propylitic assemblage and locally eliminating any acid-neutralizing capacity while introducing acid-generating minerals, especially pyrite. These assemblages are particularly evident in the vicinity of Red Mountain \#3 (fig. 2) whose slopes drain into Prospect Gulch. The summit and slopes of Red Mountain \#3 are stained with secondary mineral coatings that are caused mainly by the oxidation and weathering of pyrite. The mineral coatings impart spectacular hues of red, yellow, and brown, which led to the naming of the Red Mountains.

The geology of the area and types of alteration vary substantially from north to south across the Prospect Gulch subbasin (Bove and others, 2006). The majority of intensely altered terrain is exposed along the northern part of the subbasin on south-facing slopes along and beneath the ridge that separates Dry Gulch from Prospect Gulch (fig. 2). Acid sulfate mineralization is exposed near Red Mountain \#3 and is characterized by an acid-sulfate mineral assemblage that includes quartz, alunite, pyrophyllite (QAP assemblage), and pyrite. Pervasive silicification accompanied the acid sulfate alteration where it forms highly resistant ridges such as those exposed on Red Mountain \#3. Poorly indurated and more easily weathered argillic (ARG assemblage) alteration occurs on the margins of the QAP assemblage; dickite (a waxy clay mineral in outcrop) is commonly associated with this assemblage. Pervasive quartz-sericite-pyrite (QSP assemblage) alteration, locally containing 10-20 volume percent pyrite where not oxidized, is the largest alteration type exposed in Prospect Gulch. The QSP assemblage commonly crops out topographically below or adjacent to the QAP and ARG assemblages. Similar to the ARG assemblage, the QSP assemblage (especially near the margins) is more readily weathered than the QAP assemblage. Surficial deposits including debris cones and talus that have formed below Red Mountain \#3 incorporate clasts of the upslope QAP, ARG, and QSP assemblages.

In the southern part and lower one-third of Prospect Gulch, regional propylitic alteration dominates, although it is locally overprinted by narrow, more intensely altered zones of QAP and QSP assemblages that are exposed in northeasttrending gulleys in the upper part of the subbasin that drain toward the north into Prospect Gulch. Locally, surficial deposits involving mainly propylitically altered clasts, whose source is located along the ridge that separates Georgia Gulch from Prospect Gulch, are volumetrically important.

A general structural fabric is evident in and around Prospect Gulch based on interpretation of mapped veins (D.J. Bove, unpub. data, 2005; Bove and others, 2006). Northwest-, north-, and northeast-trending veins are evident along with another prominent set of east-west-trending veins. These veins may have formed along existing faults or formed contemporaneously with mineralization along structural zones of weakness. East-west-trending veins are not as common as their north-trending counterparts, but they do occur. Major structures, if present, are obscured largely by the intense alteration that has affected much of the northern subbasin. Additional detailed mapping is needed to determine offset of marker horizons along faults (if any are preserved) such as interbedded volcaniclastic units within the Silverton Volcanics. However, mapped offsets in the upper Prospect Gulch area are as much as $100 \mathrm{~m}$ (D.J. Bove, unpub. data, 2005) 


\section{Hydrologic Setting}

The stream in Prospect Gulch is approximately $2.4 \mathrm{~km}$ in length with an elevation change of $800 \mathrm{~m}$. Average annual precipitation is about $114 \mathrm{~cm}$ with $94 \mathrm{~cm}$ occurring as snowfall (Wirt and others, 2001). As a result, most recharge into the ground-water system occurs in late May and early June during the spring snowmelt. Late June through September is dominated by summer thunderstorms, producing rainfall that provides additional ground-water recharge (Wirt and others, 1999). Snow generally covers the ground surface in most of Prospect Gulch from October through early May, preventing any significant recharge to the ground water-system.

\section{Drilling Locations, Core Logs, and Oxidation Depth}

All drill hole locations with core data in Prospect Gulch are shown in figure 3 and table 1 . The drill core locations for UPG, MPG, and LPG (upper, middle, and lower Prospect Gulch) were drilled in 2004 as part of a Raymond Johnson's Mendenhall Postdoctoral Research Program with the USGS. Core locations labeled as M were core drilled in 1983 by Amoco Minerals (unpub. data) and locations labeled as $\mathrm{H}$ were rotary drilled by Caldedonia Resources in 1984 (unpub. data).

Original core logs for UPG, MPG, and LPG are provided in Johnson and Yager (2006). Additional detailed description of the cores for UPG, MPG, and LPG were completed as part of the sample collection process for mineralogy and are provided as appendices $\mathrm{A}$ through $\mathrm{C}$. The depths of the partially oxidized zone and the fully oxidized zone are presented in figures 4 and 5, respectively.

\section{Mineralogy from Cores}

Quantitative mineralogy was determined by X-ray diffraction (XRD) for all samples and is presented in tables 2 through 7 . Ultimately, this mineralogy data will be used to better understand the rock/water interactions that are occurring in Prospect Gulch. The samples were prepared according to the methods described by Srodon and others (2001). In summary, $3 \mathrm{~g}$ of sample were mixed with $0.333 \mathrm{~g}$ of an internal standard (zincite). The mixture was subsequently ground with $4 \mathrm{~mL}$ of methanol in a McCrone mill for 5 minutes, oven dried at $85^{\circ} \mathrm{C}$, passed through a $4 \mathrm{~mm}$ sieve, and then side-loaded into an aluminum holder to optimize random orientation. Samples were X-rayed from 5 to $65^{\circ} 2 \theta$ with $\mathrm{CuK \alpha}$ radiation $(40 \mathrm{Kv}$, $30 \mathrm{~mA}$ ) using a Siemens D500 X-ray diffraction system with a graphite monochromator, $1^{\circ}$ slits, a step size of $0.02^{\circ} 2 / \theta$, and a counting time of $2 \mathrm{~s}$ per step.
The XRD data were converted into wt $\%$ minerals using the RockJock computer software (Eberl, 2003). The program compares integrated X-ray intensities for minerals present in a sample with that of an internal zincite standard, and weight percents are calculated from previously measured mineral intensity factors (MIF's; also referred to as reference intensity ratios, RIR's). Integraged X-ray intensities for individual minerals were determined by fitting stored XRD patterns for pure mineral phases to the measured XRD pattern using the Solver option in Microsoft Excel. The Solver option minimized the degree of fit parameter between measured and calculated patterns by varying the intensities of the stored standard patterns by multiplying each of these patterns by a separate factor.

The RockJock technique has been checked for accuracy using artificial mixtures, and generally results are within 1 to $2 \mathrm{wt} \%$ of actual values (Eberl, 2003). All mineral weight percentages presented in tables 2 through 7 were normalized to $100 \%$. X-ray amorphous material was determined to be negligible due to low backgrounds in the XRD patterns and the nature of the rock samples.

\section{References Cited}

Besser, J.M., Finger, S.E., and Church, S.E., 2006, Impacts of historical mining on aquatic ecosystems-An ecological risk assessment, in Church, S.E., von Guerard, P., and Finger, S.E., eds., Integrated investigations of environmental effects of historical mining in the Animas River watershed, San Juan County, Colorado: U.S. Geological Survey Professional Paper 1651.

Bove, D.J., Hon, K., Budding, K.E., Slack, J.F., Snee, L.W., and Yeoman, R.A., 2001, Geochronology and geology of late Oligocene through Miocene volcanism and mineralization in the western San Juan Mountains, Colorado: U.S. Geological Survey Professional Paper 1642, 30 p. http:// pubs.usgs.gov/of/1999/ofr-99-0347/.

Bove, D.J., Mast, M.A., Dalton, J.B., Wright, W.G., and Yager, D.B, 2006, Major styles of mineralization and hydrothermal alteration and related solid- and aqueous-phase geochemical signatures, in Church, S.E., von Guerard, P., and Finger, S.E., eds., Integrated investigations of environmental effects of historical mining in the Animas River watershed, San Juan County, Colorado: U.S. Geological Survey Professional Paper 1651.

Burbank, W.S., 1947, Red Mountain District, Ouray County in Vanderwilt, J.W., ed., Mineral resources of Colorado: State of Colorado, Mineral Resources Board.

Burbank, W.S., 1960, Pre-ore propylitization, Silverton Caldera, Colorado, in Geological Survey Research 1960: U.S. Geological Survey Professional Paper 400-B, article 6, p. B12-B13. 
Church, S.E., von Guerard, P., and Finger, S.E., eds., 2006, Integrated investigations of environmental effects of historical mining in the Animas River watershed, San Juan County, Colorado: U.S. Geological Survey Professional Paper 1651, 6 plates, 1 DVD.

Eberl, D.D., 2003, User guide to RockJock-A program for determining quantitative mineralogy from X-ray diffraction data: U.S. Geological Survey Open-File Report 2003-78, $40 \mathrm{p}$.

Johnson, R.H., and Yager, D.B., 2006, Completion reports, core logs, and hydrogeologic data from wells and piezometers in Prospect Gulch, San Juan County, Colorado: U.S. Geological Survey Open-File Report 2006-1030, http:// pubs.usgs.gov/of/2006/1030/.

Kimball, B.A., Runkel, R.L., Walton-Day, K.W., and Bencala, K.E., 2002, Assessment of metal loads in watersheds affected by acid mine drainage by using tracer injection and synoptic sampling - Cement Creek, Colorado, USA: Applied Geochemistry, v. 17, p. 1183-1207.

Kimball, B.A., Walton-Day, K., and Runkel, R.L., 2006, Quantification of metal loading by tracer injection and synoptic sampling, 1996-2000, in Church, S.E., von Guerard, Paul, and Finger, S.E., eds., Integrated investigations of environmental effects of historical mining in the Animas River Watershed, San Juan County, Colorado: U.S. Geological Survey Professional Paper 1651.

Lipman P.W., Fisher, W.S., Mehnert, H.H., Naeser, C.W., Luedke, R.G., and Steven, T.A., 1976, Multiple ages of mid-Tertiary mineralization and alteration in the western San Juan Mountains, Colorado: Economic Geology, v. 71, p. 571-588.

Lipman, P.W., Steven, T.A., Luedke, R.G., and Burbank, W.S., 1973, Revised volcanic history of the San Juan, Uncompahgre, Silverton, and Lake City calderas in the western San Juan Mountains, Colorado: U.S. Geological Survey Journal of Research, v. 1, p. 627-642.
Manning, A.H., Solomon, D.K., and Sheldon, A.L., 2003. Applications of a total dissolved gas pressure probe in ground water studies: Ground Water, v. 41, p. 440-448.

Mast, M.A., Verplanck, P.L., Wright, W.G., and Bove, D.J., 2006, Characterization of background water quality, in Church, S.E., von Guerard, P., and Finger, S.E., eds., Integrated investigations of environmental effects of historical mining in the Animas River watershed, San Juan County, Colorado: U.S. Geological Survey Professional Paper 1651.

Srodon, J., Drits, V.A., McCarty, D.K., Hsieh, J.C., and Eberl, D.D., 2001, Quantitative mineral analysis by powder X-ray diffraction from random preparations: Clays and Clay Minerals, v. 49, p. 514-528.

Wirt, L., Leib, K.J., Bove, D.J., Mast, M.A., Evans, J.B., and Meeker, G.P., 1999, Determination of chemical-constituent loads during base-flow and storm-runoff conditions near historical mines in Prospect Gulch, upper Animas River

watershed, southwestern Colorado: U.S. Geological Survey Open-File Report 99-159, 39 p.

Wirt, L., Leib, K.J., Bove, D., and Melick, R., 2001, Metal loading assessment of point and non-point sources in a small alpine sub-basin characterized by acid drainageProspect Gulch, upper Animas River watershed, Colorado: U.S. Geological Survey Open-File Report 2001-0258, 36 p.

Yager, D.B., and Bove, D.J., 2002, Generalized geologic map of part of the upper Animas River watershed and vicinity, Silverton, Colorado: U.S. Geological Survey Miscellaneous Field Studies Map MF-2377, scale 1:48,000.

Yager, D.B., McCafferty, A.E., Stanton, M.R., Diehl, S.F., Driscoll, R.L., Fey, D.L., and Sutley, S.J., 2005, Net acid production, acid neutralizing capacity, and associated geophysical, mineralogical, and geochemical characteristics of Animas River watershed rocks near Silverton, Colorado: U.S. Geological Survey Open-File Report 2005, 78 p. 
Figure 1. 5

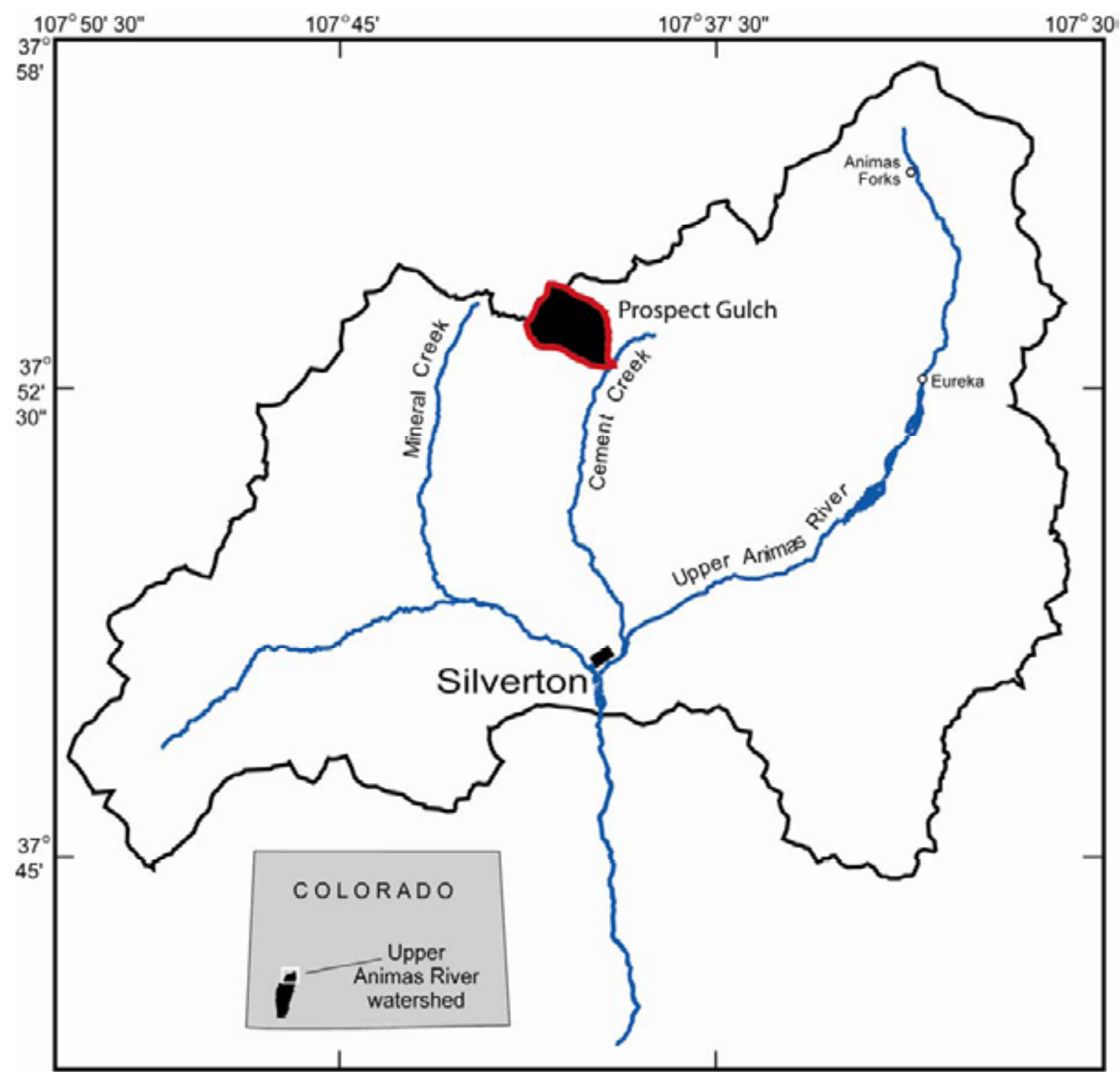

Figure 1. Location of Prospect Gulch in the upper Animas River watershed. 

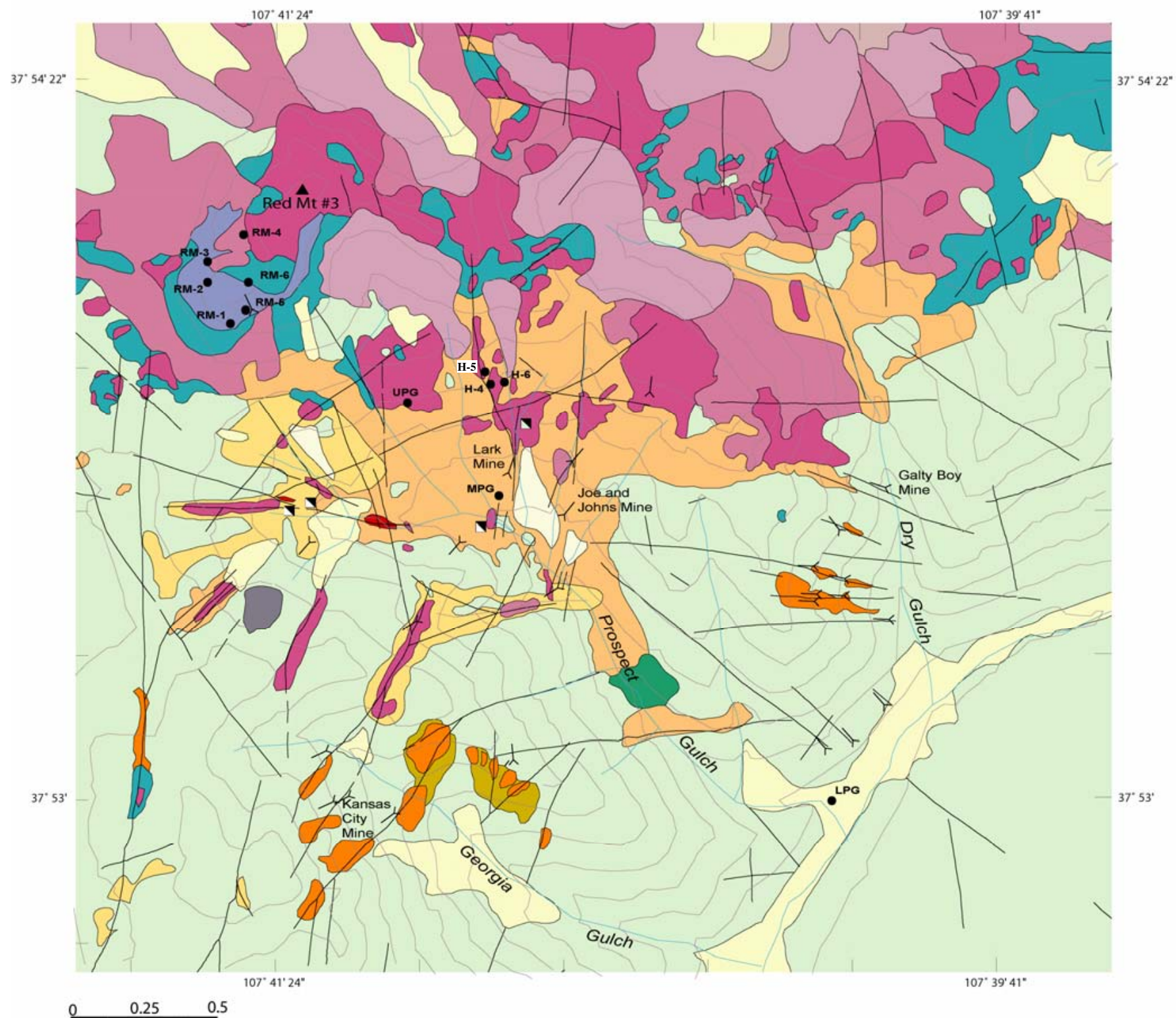

kilometers

EXPLANATION

HYDROTHERMAL ALTERATION ASSEMBLAGES

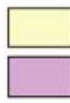

Qls- Landslide

Qu- Quatemary undivided; lacks spectral signature (AVIRIS)

Qqas- Coarser surficial deposits with strong spectra signature. Dominantly QAP and less QSP

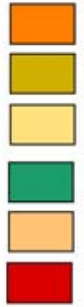

vqsp- Vein-related quartz-sericite-pyrite (QSP)

wsp-pr- Mixed weak sericite-pyrite (WSP) and propylitic (PROP)

wsp- Weak sericite-pyrite alteration

qsp-pr- Mixed QSP and PROP

qsp-Quartz-sericite-pyrite

mpy- Massive pyrite zone
Acid-Sulfate Suite

sil-silicified zone

qap- Mixed quartz-alunite (QA) and quartz-alunite-pyrophyllite ( QAP)

qap-s-Mixed QA and QAP with less QSP

arg- Argillic

pr-as- Mixed PROP and acid-sulfate (AS) suite

pr- Propylitic alteration undivided

Fault, major fracture zone, vein, silicified fault or fracure zone

Adit

- Drill hole location

Figure 2. Detailed alteration map of bedrock in Prospect Gulch and the surrounding area. 
Figure 3. $\quad 7$

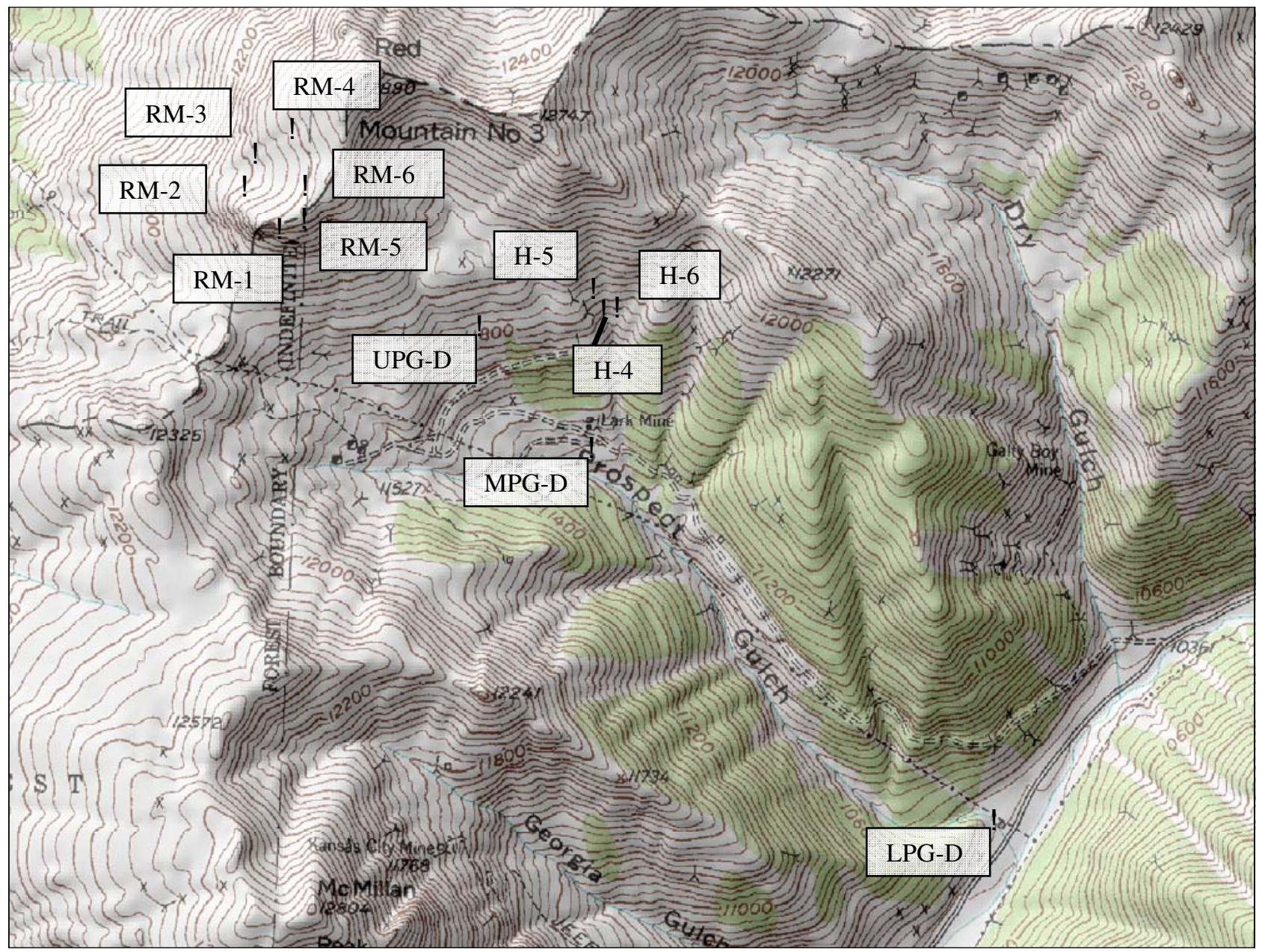

Figure 3. Drill hole locations in Prospect Gulch. 


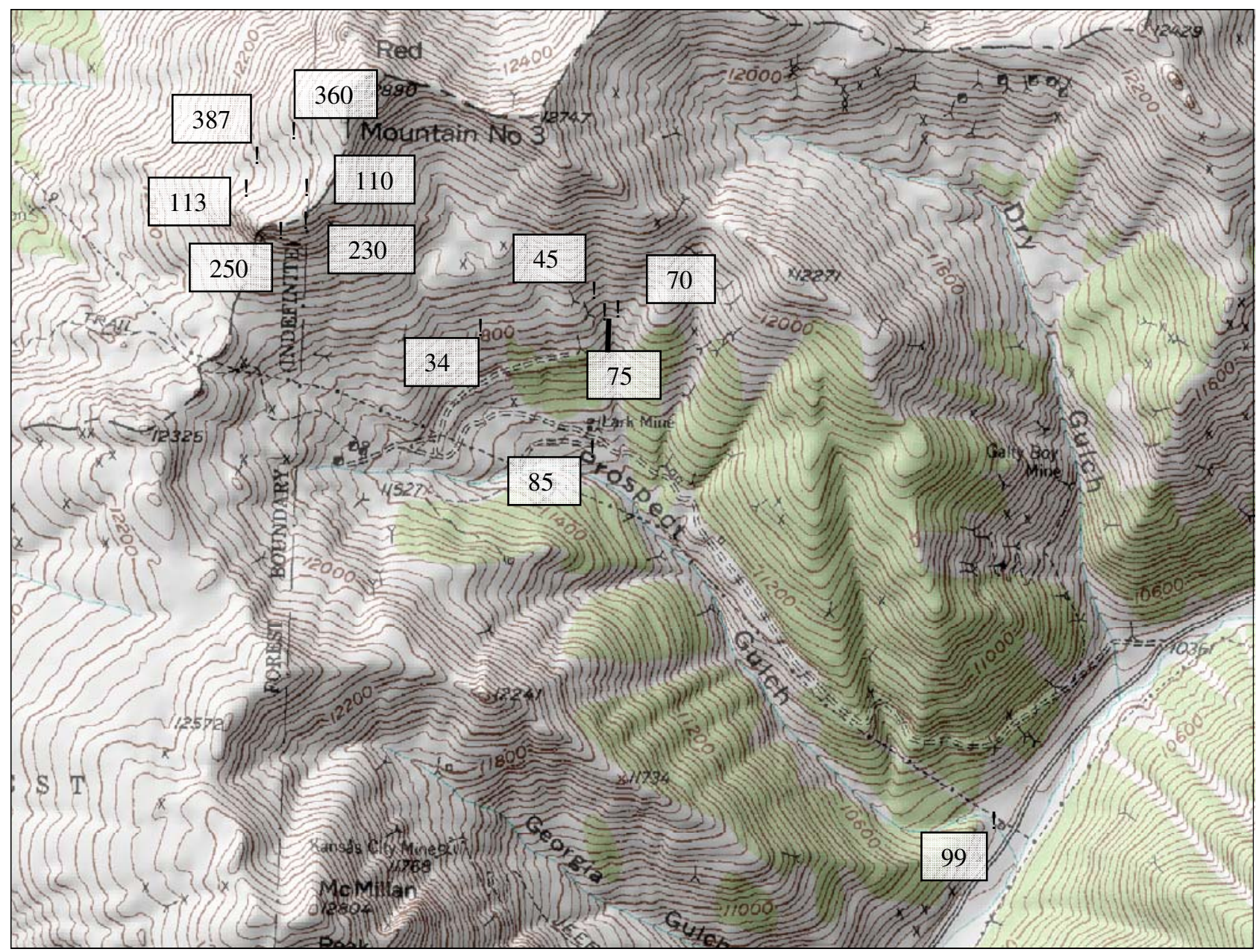

Figure 4. Depth of the partially oxidized zone in feet. (Note that the 113-foot depth of the partially oxidized zone in RM-2 is the total depth of the hole.) 
Figure 5.

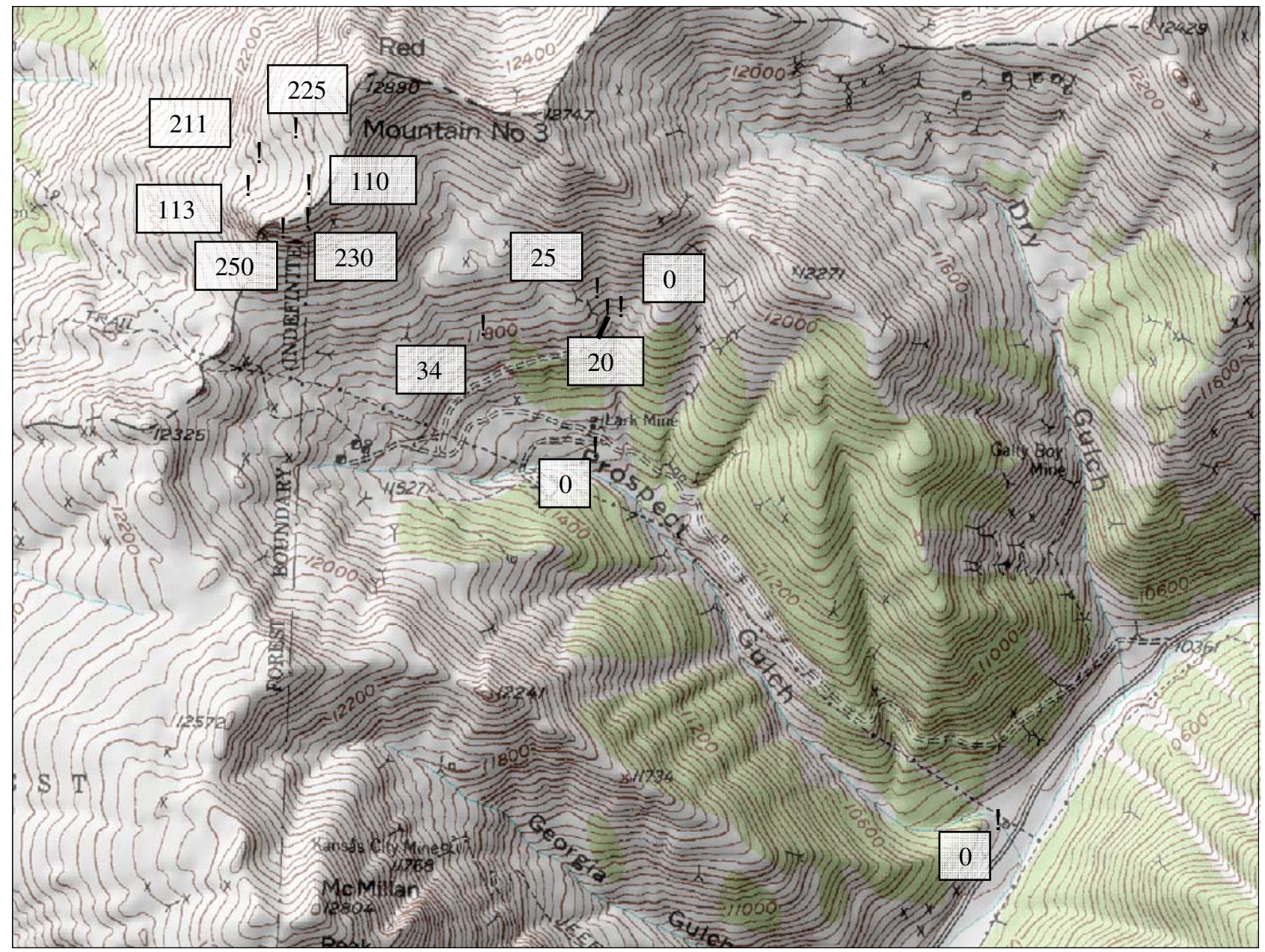

Figure 5. Depth of the fully oxidized zone in feet. (Note that the 113 -foot depth of the fully oxidized zone in RM-2 is the total depth of the hole.) 
Table 1. Drill hole locations and oxidation depths. (Note that total depth for RM-2 is 113 feet.) [ft, feet]

\begin{tabular}{ccccc}
\hline Location & $\begin{array}{c}\text { Latitude } \\
\text { (decimal } \\
\text { degrees) }\end{array}$ & $\begin{array}{c}\text { Longitude } \\
\text { (decimal } \\
\text { degrees) }\end{array}$ & $\begin{array}{c}\text { Depth of } \\
\text { partially } \\
\text { oxidized } \\
\text { zone (ft) }\end{array}$ & $\begin{array}{c}\text { Depth of } \\
\text { fully } \\
\text { oxidized } \\
\text { zone (ft) }\end{array}$ \\
\hline UPG-D & 37.8952827 & -107.6848674 & 34 & 34 \\
MPG-D & 37.8922303 & -107.6811235 & 0 & 85 \\
LPG-D & 37.8829656 & -107.6677624 & 0 & 99 \\
RM-1 & 37.8976240 & -107.6914280 & 250 & 250 \\
RM-2 & 37.8987050 & -107.6925920 & 113 & 113 \\
RM-3 & 37.8995550 & -107.6922720 & 211 & 387 \\
RM-4 & 37.9002040 & -107.6911050 & 225 & 360 \\
RM-5 & 37.8979080 & -107.6906400 & 230 & 230 \\
RM-6 & 37.8987730 & -107.6906380 & 110 & 110 \\
H-4 & 37.8957820 & -107.6808580 & 20 & 75 \\
H-5 & 37.8963210 & -107.6812110 & 25 & 45 \\
H-6 & 37.8958660 & -107.6804270 & 0 & 70 \\
\hline
\end{tabular}


Table 2. 11

Table 2. Mineralogy in UPG-D samples from X-ray diffraction analyses (sample number indicates depth in feet).

[FPF, full pattern fit]

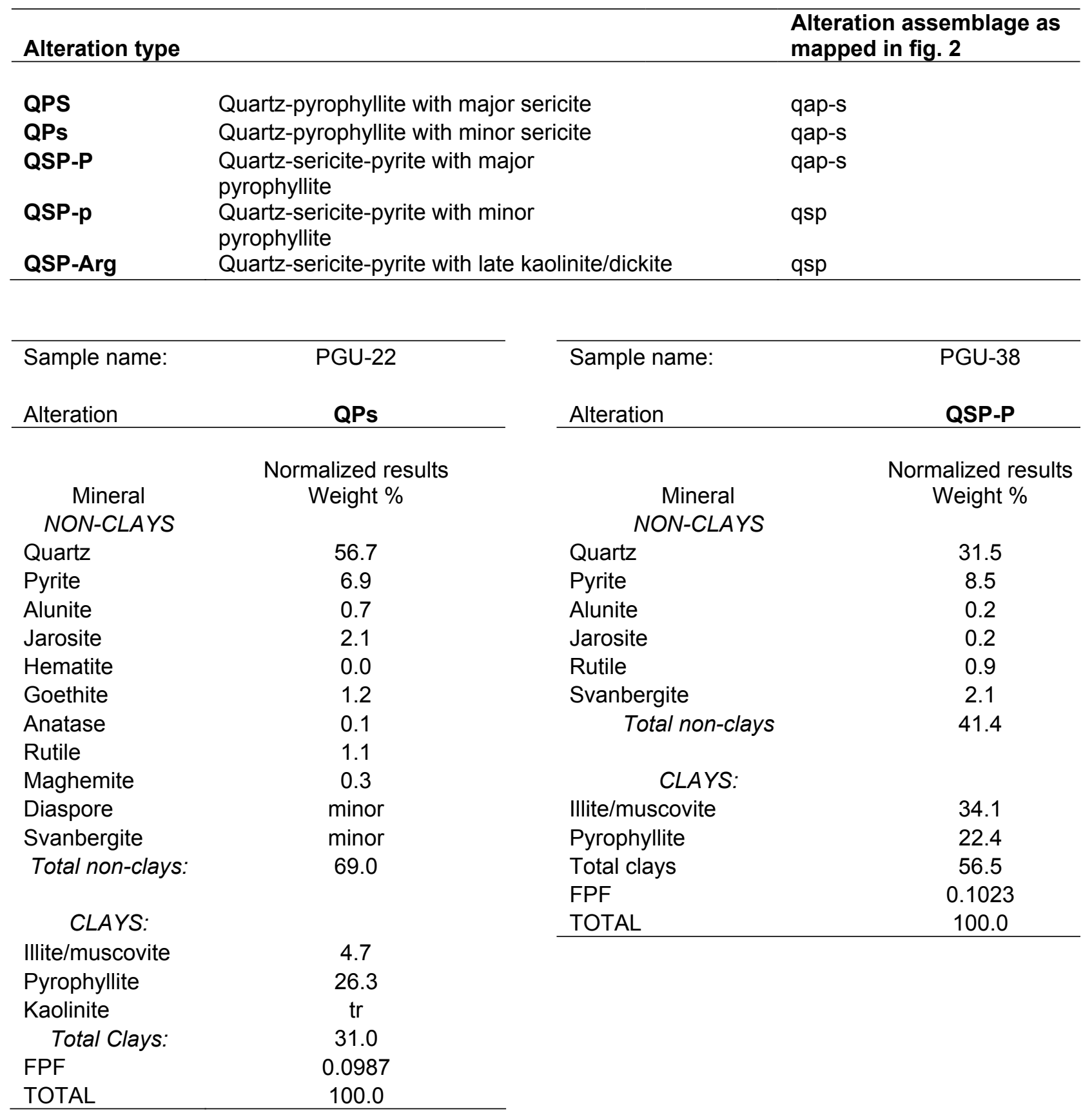


Table 2. Mineralogy in UPG-D samples-Continued.

\begin{tabular}{lc}
\hline Sample name: & PGU-40 \\
Alteration & QSP-p \\
\hline \multicolumn{1}{c}{ Mineral } & $\begin{array}{c}\text { Normalized results } \\
\text { Weight } \%\end{array}$ \\
NON-CLAYS & \\
Quartz & 55.1 \\
Pyrite & 8.4 \\
Rutile & 0.5 \\
Svanbergite & minor \\
Total non-clays: & 64.1 \\
$\quad$ CLAYS: & \\
Illite/muscovite & 31.7 \\
Pyrophyllite & 4.2 \\
Total Clays: & 35.9 \\
FPF & 0.0983 \\
TOTAL & 100.0 \\
\hline
\end{tabular}

\begin{tabular}{ll}
\hline Sample name: & PGU-47 \\
Alteration & QSP-Arg \\
\hline
\end{tabular}

\begin{tabular}{lc} 
Alteration & QSP-Arg \\
\multicolumn{1}{c}{$\begin{array}{c}\text { Mineral } \\
\text { NON-CLAYS }\end{array}$} & $\begin{array}{c}\text { Normalized results } \\
\text { Weight } \%\end{array}$ \\
Quartz & \\
Pyrite & 39.3 \\
Jarosite & 16.6 \\
Goethite & 0.3 \\
Anatase & 0.1 \\
Rutile & 0.1 \\
Svanbergite & 1.0 \\
\multicolumn{1}{c}{ Total non-clays: } & minor \\
\multicolumn{1}{c}{ CLAYS: } & 57.3 \\
Dickite/kaolinite & \\
Illite/muscovite & 11.1 \\
\multicolumn{1}{c}{ Total Clays: } & 31.7 \\
FPF & 42.7 \\
TOTAL & 0.0997 \\
\hline \multicolumn{2}{l}{ Sample name: } \\
Alteration & 100.0 \\
\hline
\end{tabular}

\begin{tabular}{lc} 
Alteration & QSP-P \\
\hline \multicolumn{1}{c}{ Mineral } & $\begin{array}{c}\text { Normalized results } \\
\text { Weight } \%\end{array}$ \\
NON-CLAYS & \\
Quartz & 43.9 \\
Pyrite & 2.3 \\
Jarosite & 1.0 \\
Goethite & 1.2 \\
Anatase & 0.1 \\
Rutile & 1.2 \\
Svanbergite & minor \\
Total non-clays: & 49.7 \\
$\quad$ CLAYS: & \\
Illite/muscovite & 37.8 \\
Pyrophyllite & 12.5 \\
$\quad$ Total Clays: & 50.3 \\
FPF & 0.0982 \\
TOTAL & 100.0 \\
\hline
\end{tabular}

\begin{tabular}{lc}
\multicolumn{1}{c}{\begin{tabular}{c} 
Mineral \\
\multicolumn{1}{c}{ NON-CLAYS }
\end{tabular}} & $\begin{array}{c}\text { Normalized results } \\
\text { Weight } \%\end{array}$ \\
Quartz & \\
Pyrite & 43.4 \\
Alunite & 9.0 \\
Jarosite & 0.2 \\
Goethite & 1.7 \\
Anatase & 0.6 \\
Rutile & 0.1 \\
Svanbergite & 0.6 \\
$\quad$ Total non-clays: & minor \\
$\quad$ CLAYS: & 55.6 \\
Dickite/kaolinite & \\
Illite/muscovite & \\
Pyrophyllite & 3.9 \\
$\quad$ Total Clays: & 12.9 \\
FPF & 27.6 \\
TOTAL & 40.4 \\
\hline
\end{tabular}


Table 2. Mineralogy in UPG-D samples-Continued.

\begin{tabular}{lc}
\hline Sample name: & PGU-125 \\
Alteration & QPS \\
\hline \multicolumn{1}{c}{ Mineral } & $\begin{array}{c}\text { Normalized results } \\
\text { Weight } \%\end{array}$ \\
NON-CLAYS & \\
Quartz & 37.2 \\
Pyrite & 19.9 \\
Alunite & 1.0 \\
Jarosite & 0.5 \\
Goethite & 0.3 \\
Rutile & 1.0 \\
Svanbergite & minor \\
Total non-clays: & 59.9 \\
$\quad$ & \\
$\quad$ CLAYS: & 1.8 \\
Dickite/kaolinite & 17.9 \\
Illite/muscovite & 22.2 \\
Pyrophyllite & 38.3 \\
$\quad$ Total Clays: & 0.0967 \\
FPF & 100.0 \\
TOTAL & \\
\hline
\end{tabular}


Table 3. Mineralogy in MPG-D samples from X-ray diffraction analyses (sample number indicates depth in feet).

[FPF, full pattern fit]

\begin{tabular}{lll}
\hline Alteration type & & $\begin{array}{l}\text { Alteration assemblage as } \\
\text { mapped in fig. 2 }\end{array}$ \\
\hline QP & Quartz-pyrophyllite & qap \\
QPS & Quartz-pyrophyllite with major & qap-s \\
& sericite & qsp \\
QSP & Quartz-sericite-pyrite & qap-s \\
QSP-P & Quartz-sericite-pyrite with major pyrophyllite & qsp \\
QSP-p & Quartz-sericite-pyrite with minor pyrophyllite & \\
\hline
\end{tabular}

\begin{tabular}{lc}
\hline Sample name: & PGM-67-70 \\
Alteration & QSP-P \\
\hline \multicolumn{1}{c}{ Mineral } & Normalized results \\
$\quad$ NON-CLAYS & Weight $\%$ \\
Quartz & \\
Anhydrite & 49.8 \\
Gypsum & 0.1 \\
Goethite & 0.7 \\
Rutile & 0.1 \\
Pyrite & 1.1 \\
Barite & 9.0 \\
Alunite & 1.6 \\
Jarosite & 0.5 \\
Diaspore & 3.8 \\
Sphalerite & 4.9 \\
Svanbergite & 3.9 \\
Total non-clays & 0.6 \\
$\quad$ & 76.1 \\
$\quad$ CLAYS: & \\
disordered & \\
Kaolinite & \\
ordered Kaolinite & \\
Pyrophyllite & 0.1 \\
Illite/muscovite & 2.4 \\
$\quad$ Total clays & 18.0 \\
FPF & 27.3 \\
TOTAL & 23.9 \\
\hline
\end{tabular}

\begin{tabular}{lc}
\hline Sample name: & PGM-80 \\
Alteration & QSP-P \\
\hline
\end{tabular}

Mineral
NON-CLAYS

Quartz

Anhydrite

Gypsum

Goethite

Maghemite

Rutile

Pyrite

Barite

Alunite

Jarosite

Diaspore

Svanbergite

Total non-clays

Normalized results

Weight \%

45.2

0.2

0.7

0.9

0.4

0.9

7.1

0.6

0.3

1.8

8.0

1.4

67.6

\begin{tabular}{lc}
$\quad$ CLAYS: & \\
disordered & \\
Kaolinite & 4.3 \\
ordered Kaolinite & 2.6 \\
Pyrophyllite & 22.8 \\
Illite/muscovite & 2.6 \\
$\quad$ Total clays & 32.5 \\
FPF & 0.0992 \\
TOTAL & 100 \\
\hline
\end{tabular}


Table 3. $\quad 15$

Table 3. Mineralogy in MPG-D—Continued.

\begin{tabular}{|c|c|c|c|}
\hline $\begin{array}{l}\text { Sample } \\
\text { name: }\end{array}$ & PGM-88 & Sample name: & PGM-100 \\
\hline Alteration & QP & Alteration & QSP-P \\
\hline $\begin{array}{c}\text { Mineral } \\
N O N-C L A Y S\end{array}$ & $\begin{array}{c}\text { Normalized results } \\
\text { Weight } \%\end{array}$ & $\begin{array}{c}\text { Mineral } \\
\text { NON-CLAYS }\end{array}$ & $\begin{array}{c}\text { Normalized results } \\
\text { Weight } \%\end{array}$ \\
\hline Quartz & 45.5 & Quartz & 42.4 \\
\hline Gypsum & 0.3 & Pyrite & 8.0 \\
\hline Goethite & 0.2 & Anhydrite & 0.1 \\
\hline Rutile & 0.7 & Goethite & 0.8 \\
\hline Pyrite & 5.1 & Anatase & 0.6 \\
\hline Barite & 0.4 & Svanbergite & 1.0 \\
\hline Alunite & 0.0 & Total non-clays & 52.9 \\
\hline Jarosite & 0.2 & & \\
\hline Diaspore & 2.1 & CLAYS: & \\
\hline & 1.0 & Illite/muscovite & 34.0 \\
\hline \multirow{3}{*}{$\begin{array}{l}\text { Total non- } \\
\text { clays }\end{array}$} & 55.5 & Pyrophyllite & 13.1 \\
\hline & & Total clays & 47.1 \\
\hline & & FPF & 0.1033 \\
\hline CLAYS: & & TOTAL & 100.0 \\
\hline Pyrophyllite & 44.5 & & \\
\hline Total clays & 44.5 & & \\
\hline FPF & 0.0979 & & \\
\hline TOTAL & 100 & & \\
\hline
\end{tabular}


Table 3. Mineralogy in MPG-D_Continued.

\begin{tabular}{|c|c|c|c|}
\hline Sample name: & PGM-108 & Sample name: & PGM-119 \\
\hline \multirow[t]{2}{*}{ Alteration } & QSP-p & Alteration & QP \\
\hline & Normalized results & & Normalized results \\
\hline Mineral & Weight \% & Mineral & Weight \% \\
\hline NON-CLAYS & & NON-CLAYS & \\
\hline Quartz & 37.1 & Quartz & 18.3 \\
\hline Pyrite & 4.8 & Gypsum & 0.3 \\
\hline Anhydrite & 0.3 & Goethite & 0.2 \\
\hline Gypsum & 0.3 & Pyrite & 12.0 \\
\hline Jarosite & 0.2 & $\begin{array}{l}\text { Jarosite } \\
\quad \text { Total non- }\end{array}$ & 0.2 \\
\hline Goethite & 0.5 & clays & 31.1 \\
\hline Anatase & 0.2 & & \\
\hline Rutile & 0.3 & CLAYS: & \\
\hline Svanbergite & 1.4 & Pyrophyllite & 68.9 \\
\hline \multirow{3}{*}{$\begin{array}{c}\text { Total non- } \\
\text { clays }\end{array}$} & & & \\
\hline & 45.1 & Total clays & 68.9 \\
\hline & & FPF & 0.1012 \\
\hline CLAYS & & TOTAL & 100.0 \\
\hline Illite/muscovite & 47.8 & & \\
\hline Pyrophyllite & 7.1 & & \\
\hline Total clays & 54.9 & & \\
\hline FPF & 0.1011 & & \\
\hline TOTAL & 100.0 & & \\
\hline
\end{tabular}


Table 3. 17

Table 3. Mineralogy in MPG-D—Continued.

\begin{tabular}{lc}
\hline Sample name: & PGM-133 \\
Alteration & QSP \\
\hline Mineral & $\begin{array}{c}\text { Normalized results } \\
\text { Weight } \%\end{array}$ \\
NON-CLAYS & \\
Quartz & 48.5 \\
Anhydrite & 0.2 \\
Gypsum & 0.4 \\
Goethite & 0.4 \\
Rutile & 0.7 \\
Pyrite & 7.4 \\
Barite & 0.3 \\
Alunite & 0.2 \\
Jarosite & 1.2 \\
Total non- & \\
$\quad$ clays & 59.3 \\
CLAYS: & \\
Illite/muscovite & 40.7 \\
Total clays & 40.7 \\
FPF & 0.0999 \\
TOTAL & 100.0 \\
\hline
\end{tabular}

\begin{tabular}{lc}
\hline Sample name: & PGM-143 \\
Alteration & QSP-P \\
\hline \multicolumn{1}{c}{ Mineral } & $\begin{array}{c}\text { Normalized results } \\
\text { Weight } \%\end{array}$ \\
NON-CLAYS & \\
Quartz & 46.6 \\
Anhydrite & 0.2 \\
Gypsum & 0.1 \\
Maghemite & 0.3 \\
Anatase & 0.3 \\
Rutile & 0.3 \\
Pyrite & 10.4 \\
Alunite & 0.6 \\
Svanbergite & 1.7 \\
& \\
Total non-clays & 58.8 \\
& \\
CLAYS: & \\
Illite/muscovite & 26.5 \\
Pyrophyllite & 13.0 \\
Total clays & 39.4 \\
FPF & 0.0937 \\
TOTAL & 100.0 \\
\hline
\end{tabular}


Table 3. Mineralogy in MPG-D_Continued.

\begin{tabular}{lc}
\hline Sample name: & PGM-157 \\
Alteration & QSP-P \\
\hline \multicolumn{1}{c}{ Mineral } & $\begin{array}{c}\text { Normalized results } \\
\text { Weight } \%\end{array}$ \\
NON-CLAYS & \\
Quartz & 35.5 \\
Pyrite & 11.8 \\
Anhydrite & 0.5 \\
Bassanite & 0.1 \\
Alunite & 0.3 \\
Goethite & 0.4 \\
Anatase & 0.1 \\
Rutile & 0.7 \\
IImenite & 0.1 \\
Svanbergite & 2.5 \\
Total non- & \\
$\quad$ clays & 52.1 \\
& \\
CLAYS: & \\
Illite/muscovite & 37.4 \\
Pyrophyllite & 10.4 \\
Total clays & 47.9 \\
FPF & 0.0988 \\
TOTAL & 100.0 \\
\hline \multicolumn{2}{c}{} \\
\hline
\end{tabular}

\begin{tabular}{lc}
\hline Sample name: & PGM-166 \\
Alteration & QSP \\
\hline \multicolumn{1}{c}{ Mineral } & $\begin{array}{c}\text { Normalized results } \\
\text { Weight } \%\end{array}$ \\
Quartz & \\
Anhydrite & 36.9 \\
Gypsum & 0.5 \\
Goethite & 0.5 \\
Maghemite & 0.3 \\
Rutile & 0.3 \\
Pyrite & 0.8 \\
Barite & 11.2 \\
Alunite & 0.3 \\
Total non-clays & 0.1 \\
& 50.9 \\
CLAYS: & \\
disordered & \\
Kaolinite & \\
Illite/muscovite & \\
$\quad$ Total clays & 1.1 \\
FPF & 48.0 \\
TOTAL & 49.1 \\
\hline & 0.0967 \\
& 100.0 \\
\hline
\end{tabular}


Table 3. 19

Table 3. Mineralogy in MPG-D-Continued.

\begin{tabular}{lc}
\hline Sample name: & PGM-171 \\
Alteration & QSP-p \\
\hline \multicolumn{1}{c}{ Mineral } & Normalized results \\
NON-CLAYS & Weight $\%$ \\
Quartz & 37.7 \\
Anhydrite & 0.3 \\
Goethite & 0.3 \\
Anatase & 0.1 \\
Rutile & 0.4 \\
Pyrite & 12.1 \\
Barite & 0.4 \\
Alunite & 0.2 \\
Total non-clays & 51.5 \\
$\quad$ & \\
$\quad$ CLAYS: & \\
disordered & \\
Kaolinite & \\
Illite/muscovite & \\
Pyrophyllite & \\
Total clays & 43.4 \\
FPF & 5.0 \\
TOTAL & 48.5 \\
\hline$\quad$ & 0.1017 \\
\hline
\end{tabular}


Table 4. Mineralogy in LPG-D samples from X-ray diffraction analyses (sample number indicates depth in feet).

[FPF, full pattern fit]

\begin{tabular}{lll}
\hline Alteration type & & $\begin{array}{l}\text { Alteration assemblage } \\
\text { as mapped in fig. 2 }\end{array}$ \\
\hline PROP $^{\mathrm{A}}$ & Propylitic with secondary albitization & $\mathbf{p r}$ \\
WSP-PROP $^{\text {WSP-PROP }}{ }^{\mathrm{A}}$-WSP & Propylitic to weak sericite pyrite & wsp-pr \\
WSPylitic (with secondary albitization) grading to weak sericitic & wsp-pr \\
WSP-QSP & Weak sericite pyrite to quartz-sericite pyrite & $\mathbf{w s p - p r}$ \\
QSP & Quartz-sericite-pyrite & $\mathbf{q s p}$
\end{tabular}

\begin{tabular}{lc}
\hline Sample name: & PGL-13 \\
Alteration & $\begin{array}{c}\text { WSP-PROP (clast in } \\
\text { ferricrete) }\end{array}$ \\
\hline \multicolumn{1}{c}{ Mineral } & Normalized \\
$\quad$ NON-CLAYS & Weight \% \\
Quartz & \\
Albite feldspar & 34.9 \\
Andesine feldspar & 4.0 \\
Gypsum & 2.3 \\
Jarosite & 0.5 \\
Goethite & 2.0 \\
Maghemite & 9.0 \\
Apatite & 1.7 \\
Anatase & 0.6 \\
Rutile & 13.0 \\
$\quad$ Total non-clays & 0.3 \\
$\quad$ CLAYS: & 68.4 \\
Illite/muscovite & \\
$\quad$ Total clays & \\
FPF & 31.6 \\
TOTAL & 31.6 \\
\hline \multicolumn{2}{c}{} \\
\hline
\end{tabular}

\begin{tabular}{|c|c|}
\hline Sample name: & PGL-87.5 \\
\hline Alteration & WSP-PROP \\
\hline $\begin{array}{c}\text { Mineral } \\
\text { NON-CLAYS }\end{array}$ & $\begin{array}{l}\text { Normalized } \\
\text { Weight \% }\end{array}$ \\
\hline Quartz & 58.7 \\
\hline Gypsum & 0.2 \\
\hline Bassanite & 0.2 \\
\hline Jarosite & 0.5 \\
\hline Sanidine feldspar & 10.0 \\
\hline Orthoclase feldspar & 3.3 \\
\hline Albite feldspar & 11.3 \\
\hline Andesine feldspar & 0.5 \\
\hline Pyrite & 0.5 \\
\hline Total non-clays & 85.2 \\
\hline CLAYS & \\
\hline disordered Kaolinite & 2.5 \\
\hline Biotite & 0.1 \\
\hline Illite/muscovite & 10.5 \\
\hline Chlorite & 1.6 \\
\hline Total clays & 14.8 \\
\hline FPF & 0.0996 \\
\hline TOTAL & 100.0 \\
\hline
\end{tabular}


Table 4. Mineralogy in LPG-D—Continued.

\begin{tabular}{|c|c|}
\hline Sample name: & PGL-93 \\
\hline Alteration & WSP-PROP ${ }^{A}$ \\
\hline $\begin{array}{c}\text { Mineral } \\
\text { NON-CLAYS }\end{array}$ & $\begin{array}{l}\text { Normalized } \\
\text { Weight \% }\end{array}$ \\
\hline Quartz & 34.5 \\
\hline Gypsum & 0.3 \\
\hline Bassanite & 0.1 \\
\hline Jarosite & 0.2 \\
\hline Epidote & 1.1 \\
\hline Sanidine feldspar & 7.3 \\
\hline Orthoclase feldspar & 4.0 \\
\hline Albite feldspar & 33.1 \\
\hline Andesine feldspar & 0.3 \\
\hline Pyrite & 0.4 \\
\hline Total non-clays & 81.3 \\
\hline CLAYS & \\
\hline disordered Kaolinite & 1.6 \\
\hline Illite/muscovite & 10.9 \\
\hline Chlorite & 6.1 \\
\hline Total clays & 18.7 \\
\hline FPF & 0.1001 \\
\hline TOTAL & 100.0 \\
\hline
\end{tabular}

\begin{tabular}{|c|c|}
\hline Sample name: & PGL-103 \\
\hline Alteration & WSP-PROP \\
\hline Mineral & $\begin{array}{l}\text { Normalized } \\
\text { Weight \% }\end{array}$ \\
\hline NON-CLAYS & \\
\hline Quartz & 36.8 \\
\hline Gypsum & 0.1 \\
\hline Bassanite & 0.3 \\
\hline Epidote & 2.0 \\
\hline Sanidine feldspar & 21.3 \\
\hline Orthoclase feldspar & 4.2 \\
\hline Albite feldspar & 10.9 \\
\hline Pyrite & 2.1 \\
\hline Total non-clays & 77.7 \\
\hline CLAYS & \\
\hline disordered Kaolinite & 3.6 \\
\hline Biotite & 0.3 \\
\hline Illite/muscovite & 13.2 \\
\hline Chlorite & 5.2 \\
\hline Total clays & 22.3 \\
\hline FPF & 0.1017 \\
\hline TOTAL & 100.0 \\
\hline
\end{tabular}


22 Mineralogy from Cores in Prospect Gulch, San Juan County, Colorado

Table 4. Mineralogy in LPG-D—Continued.

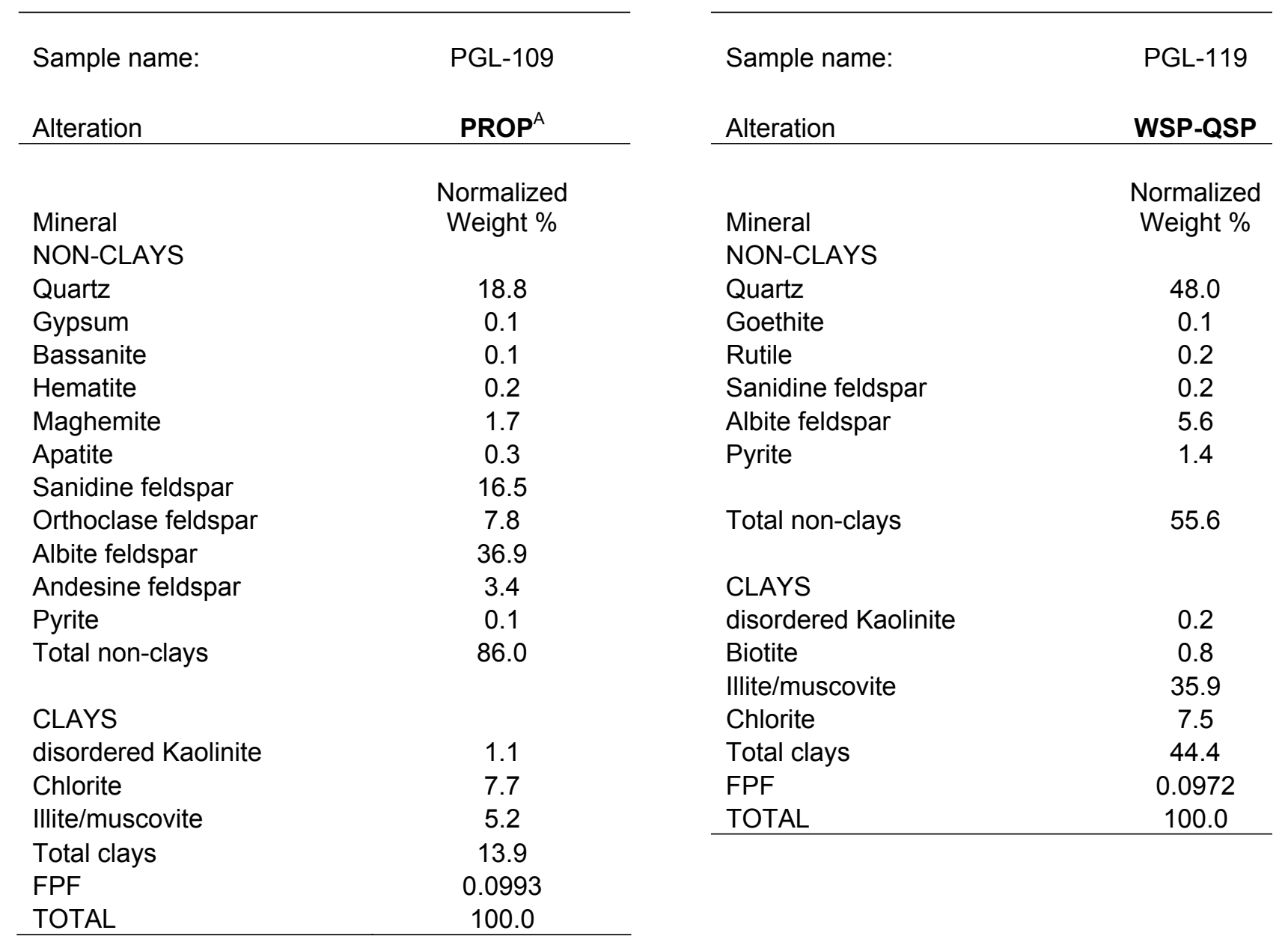


Table $4 . \quad 23$

Table 4. Mineralogy in LPG-D—Continued.

\begin{tabular}{lc}
\hline Sample name: & PGL-129 \\
Alteration & WSP-QSP \\
\hline & Normalized \\
Mineral & Weight $\%$ \\
NON-CLAYS & \\
Quartz & 42.2 \\
Goethite & 0.4 \\
Rutile & 0.3 \\
Orthoclase feldspar & 0.4 \\
Albite feldspar & 5.1 \\
Pyrite & 4.4 \\
& \\
Total non-clays & 52.7 \\
CLAYS & \\
disordered Kaolinite & \\
Illite/muscovite & 10.3 \\
Chlorite (CCa-1) & 29.6 \\
Total clays & 7.6 \\
FPF & 47.4 \\
TOTAL & 0.0999 \\
\hline
\end{tabular}

Sample name:

PGL-136.5

\begin{tabular}{lc} 
Alteration & WSP-QSP \\
\hline & Normalized Weight \\
Mineral & $\%$ \\
NON-CLAYS & \\
Quartz & 44.1 \\
Albite feldspar & 11.5 \\
Pyrite & 5.1 \\
Rutile & 0.2 \\
Total non-clays & 60.8 \\
& \\
CLAYS: & \\
Illite/muscovite & 35.0 \\
Chlorite (CCa-1) & 4.2 \\
Total clays & 39.2 \\
FPF & 0.1136 \\
TOTAL & 100.0 \\
\hline
\end{tabular}


24 Mineralogy from Cores in Prospect Gulch, San Juan County, Colorado

Table 4. Mineralogy in LPG-D—Continued.

\begin{tabular}{lc}
\hline Sample name: & PGL-142.5 \\
Alteration & QSP \\
\hline Mineral & $\begin{array}{c}\text { Normalized } \\
\text { Weight } \%\end{array}$ \\
NON-CLAYS & \\
Quartz & 52.9 \\
Pyrite & 2.0 \\
Rutile & 0.2 \\
Total non-clays & 55.1 \\
& \\
CLAYS: & \\
disordered & \\
Kaolinite & 3.1 \\
Illite/muscovite & 33.6 \\
Chlorite (CCa-1) & 8.1 \\
Chlorite (CCa-3) & 1.2 \\
Total clays & 44.9 \\
FPF & 0.1004 \\
TOTAL & 100.0 \\
\hline
\end{tabular}

\begin{tabular}{lc}
\hline Sample name: & PGL-149 \\
Alteration & WSP-QSP \\
\hline & Normalized \\
Mineral & Weight \% \\
NON-CLAYS & \\
Quartz & 45.2 \\
Goethite & 0.2 \\
Maghemite & 0.0 \\
Rutile & 0.1 \\
Sanidine feldspar & 6.6 \\
Orthoclase feldspar & 2.6 \\
& \\
Albite feldspar & 8.3 \\
Pyrite & 2.2 \\
Total non-clays & 65.1 \\
& \\
CLAYS & \\
disordered Kaolinite & 8.5 \\
Illite/muscovite & 23.8 \\
Chlorite (CCa-1) & 2.6 \\
Total clays & 34.9 \\
FPF & 0.1002 \\
TOTAL & 100.0 \\
\hline
\end{tabular}


Table 4. Mineralogy in LPG-D—Continued.

\begin{tabular}{lc}
\hline Sample name: & PGL-159 \\
Alteration & WSP-PROP \\
\hline & Normalized \\
Mineral & Weight \% \\
NON-CLAYS & \\
Quartz & 33.5 \\
Bassanite & 0.3 \\
Magnetite & 0.2 \\
Rutile & 0.4 \\
Sanidine feldspar & 8.9 \\
Orthoclase feldspar & 3.6 \\
Albite feldspar & 39.6 \\
Andesine feldspar & 3.3 \\
Pyrite & 1.2 \\
Total non-clays & 80.9 \\
& \\
CLAYS & \\
disordered Kaolinite & 1.5 \\
Biotite & 8.2 \\
Illite/Muscovite & 5.2 \\
Chlorite & 4.2 \\
Total clays & 19.1 \\
FPF & 0.0997 \\
TOTAL & 100.0 \\
\hline
\end{tabular}


Table 5. Mineralogy in H-4 samples from X-ray diffraction analyses (sample number indicates depth in feet).

[FPF, full pattern fit]

\begin{tabular}{lll}
\hline $\begin{array}{l}\text { Alteration } \\
\text { type }\end{array}$ & & $\begin{array}{l}\text { Alteration assemblage } \\
\text { as mapped in fig. 2 }\end{array}$ \\
\hline & Quartz-alunite-pyrophyllite & qap \\
QAP & Quartz-alunite with minor pyrophyllite & qap \\
QAp & Pyrophyllitic with minor alunite & qap \\
Pyroph-a & Quartz-sericite-pyrite with minor pyrophyllite & qsp \\
QSP-p & Quartz-pyrophyllite with major sericite & qap-s \\
QPS & & \\
\hline
\end{tabular}

\begin{tabular}{lc}
\hline Sample name: & H4-164 \\
Lithology & $\begin{array}{c}\text { Dark clast-supported } \\
\text { breccia } \\
\text { QAp }\end{array}$ \\
Alteration & Normalized results \\
\multicolumn{1}{c}{ Weight $\%$} \\
$\quad$ Mineral & \\
NON-CLAYS & 51.0 \\
Quartz & 1.8 \\
Anhydrite & 0.0 \\
Gypsum & 0.0 \\
Jarosite & 0.0 \\
Magnetite & 0.0 \\
Hematite & 1.9 \\
Maghemite & 0.0 \\
Anatase & 0.7 \\
Rutile & 10.9 \\
Pyrite & 14.6 \\
Alunite & 9.3 \\
Natroalunite & 3.0 \\
Diaspore & 1.7 \\
Svanbergite & \\
$\quad$ Total non- & \\
$\quad$ clays & \\
CLAYS & \\
Pyrophyllite & \\
Total clays & \\
FPF & \\
TOTAL & 5.3 \\
\hline
\end{tabular}

Sample name: $\quad \mathrm{H} 4-166$

\begin{tabular}{lc} 
Lithology & Medium gray lavas \\
Alteration & QAp \\
\hline
\end{tabular}

$\begin{array}{lc}\text { Mineral } & \begin{array}{c}\text { Normalized results } \\ \text { Weight \% }\end{array} \\ \text { NON-CLAYS } & \\ \text { Quartz } & 60.4 \\ \text { Anhydrite } & 2.1 \\ \text { Bassanite } & 0.5 \\ \text { Goethite } & 0.1 \\ \text { Maghemite } & 1.4 \\ \text { Anatase } & 0.0 \\ \text { Rutile } & 1.1 \\ \text { Pyrite } & 4.8 \\ \text { Alunite } & 17.2 \\ \text { Natroalunite } & 6.4 \\ \text { Total non-clays } & 94.0 \\ \quad & \\ \quad \text { CLAYS } & \\ \text { Pyrophyllite } & 6.0 \\ \quad \text { Total clays } & 6.0 \\ \text { FPF } & 0.0993 \\ \text { TOTAL } & 100\end{array}$


Table 5. Mineralogy in H-4-Continued.

\begin{tabular}{lll}
\hline $\begin{array}{l}\text { Alteration } \\
\text { type }\end{array}$ & & $\begin{array}{l}\text { Alteration assemblage } \\
\text { as mapped in fig. 2 }\end{array}$ \\
\hline & Quartz-alunite-pyrophyllite & qap \\
QAP & Quartz-alunite with minor pyrophyllite & qap \\
QAp & Pyrophyllitic with minor alunite & qap \\
Pyroph-a & Quartz-sericite-pyrite with minor pyrophyllite & qsp \\
QSP-p & Quartz-pyrophyllite with major sericite & qap-s \\
QPS & &
\end{tabular}

\begin{tabular}{|c|c|c|c|}
\hline Sample name: & $\mathrm{H} 4-164$ & Sample name: & H4-166 \\
\hline $\begin{array}{l}\text { Lithology } \\
\text { Alteration }\end{array}$ & $\begin{array}{c}\text { Dark clast-supported } \\
\text { breccia } \\
\text { QAp } \\
\end{array}$ & $\begin{array}{l}\text { Lithology } \\
\text { Alteration }\end{array}$ & $\begin{array}{c}\text { Medium gray lavas } \\
\text { QAp }\end{array}$ \\
\hline $\begin{array}{c}\text { Mineral } \\
N O N-C L A Y S\end{array}$ & $\begin{array}{c}\text { Normalized results } \\
\text { Weight } \%\end{array}$ & $\begin{array}{c}\text { Mineral } \\
\text { NON-CLAYS }\end{array}$ & $\begin{array}{c}\text { Normalized results } \\
\text { Weight } \%\end{array}$ \\
\hline Quartz & 51.0 & Quartz & 60.4 \\
\hline Anhydrite & 1.8 & Anhydrite & 2.1 \\
\hline Gypsum & 0.0 & Bassanite & 0.5 \\
\hline Jarosite & 0.0 & Goethite & 0.1 \\
\hline Magnetite & 0.0 & Maghemite & 1.4 \\
\hline Hematite & 0.0 & Anatase & 0.0 \\
\hline Maghemite & 1.9 & Rutile & 1.1 \\
\hline Anatase & 0.0 & Pyrite & 4.8 \\
\hline Rutile & 0.7 & Alunite & 17.2 \\
\hline Pyrite & 10.9 & Natroalunite & 6.4 \\
\hline Alunite & 14.6 & Total non-clays & 94.0 \\
\hline Natroalunite & 9.3 & & \\
\hline Diaspore & 3.0 & CLAYS & \\
\hline $\begin{array}{l}\text { Svanbergite } \\
\text { Total non- }\end{array}$ & 1.7 & Pyrophyllite & 6.0 \\
\hline clays & & Total clays & 6.0 \\
\hline & 94.8 & FPF & 0.0993 \\
\hline CLAYS & & TOTAL & 100 \\
\hline Pyrophyllite & 5.3 & & \\
\hline Total clays & 5.3 & & \\
\hline FPF & 0.0971 & & \\
\hline TOTAL & 100 & & \\
\hline
\end{tabular}


Table 5. Mineralogy in H-4-Continued.

\begin{tabular}{|c|c|}
\hline Sample name: & $\mathrm{H} 4-267$ \\
\hline $\begin{array}{l}\text { Lithology } \\
\text { Alteration }\end{array}$ & $\begin{array}{c}\text { Dark matrix-supported } \\
\text { breccia } \\
\text { Pryoph-a } \\
\end{array}$ \\
\hline $\begin{array}{c}\text { Mineral } \\
N O N-C L A Y S\end{array}$ & $\begin{array}{l}\text { Normalized results } \\
\text { Weight } \%\end{array}$ \\
\hline Quartz & 1.9 \\
\hline Anhydrite & 0.1 \\
\hline Gypsum & 0.1 \\
\hline Jarosite & 0.0 \\
\hline Magnetite & 0.6 \\
\hline Hematite & 0.2 \\
\hline Maghemite & 0.0 \\
\hline Anatase & 0.0 \\
\hline Rutile & 0.8 \\
\hline Pyrite & 16.2 \\
\hline Alunite & 6.7 \\
\hline Svanbergite & 2.2 \\
\hline Total non-clays & 26.7 \\
\hline CLAYS & \\
\hline Pyrophyllite & 71.2 \\
\hline Total clays & 71.2 \\
\hline FPF & 0.1124 \\
\hline TOTAL & 100 \\
\hline
\end{tabular}

\begin{tabular}{lc}
\hline Sample name: & H4-580 \\
Lithology & $\begin{array}{c}\text { Dark clast and matrix-supported } \\
\text { breccia } \\
\text { QAp }\end{array}$ \\
Alteration & Normalized results \\
Weight \% \\
$\quad$ Mineral & 45.3 \\
NON-CLAYS & 1.0 \\
Quartz & 0.2 \\
Maghemite & 0.7 \\
Anatase & 7.6 \\
Rutile & 27.1 \\
Pyrite & 11.5 \\
Alunite & 1.0 \\
Natroalunite & minor \\
Svanbergite & \\
Zunyite & 95.7 \\
Total non- & \\
clays & \\
CLAYS & 4.3 \\
Pyrophyllite & 4.3 \\
Total clays & 0.1061 \\
FPF & 100 \\
TOTAL & \\
\hline
\end{tabular}


Table 5. Mineralogy in H-4-Continued.

\begin{tabular}{lc}
\hline Sample name: & H4-602 \\
Lithology & $\begin{array}{c}\text { Dark clast-supported } \\
\text { breccia } \\
\text { QAp }\end{array}$ \\
Alteration & Normalized results \\
& Weight \% \\
$\quad$ Mineral & \\
NON-CLAYS & 54.8 \\
Quartz & 2.2 \\
Anhydrite & 0.0 \\
Gypsum & 0.0 \\
Jarosite & 0.0 \\
Magnetite & 0.0 \\
Hematite & 1.8 \\
Maghemite & 0.0 \\
Anatase & 0.6 \\
Rutile & 6.3 \\
Pyrite & 11.5 \\
Alunite & 14.9 \\
Natroalunite & 2.5 \\
Svanbergite & 94.7 \\
Total non-clays & \\
& \\
CLAYS & 5.3 \\
Pyrophyllite & 5.3 \\
Total clays & 100 \\
FPF & \\
TOTAL & \\
\hline
\end{tabular}

\begin{tabular}{lc}
\hline Sample name: & H4-649 \\
Lithology & Dark breccia; feint texture \\
Alteration & QAp \\
\hline \multicolumn{1}{c}{ Mineral } & Weight \% \\
NON-CLAYS & \\
Quartz & 52.3 \\
Anhydrite & 1.8 \\
Maghemite & 2.2 \\
Anatase & 0.0 \\
Rutile & 0.7 \\
Pyrite & 12.7 \\
Alunite & 10.5 \\
Natroalunite & 12.3 \\
Svanbergite & 1.8 \\
Total non-clays & 94.9 \\
& \\
$\quad$ CLAYS & \\
Pyrophyllite & 5.1 \\
Total clays & 5.1 \\
FPF & 0.1009 \\
TOTAL & 100 \\
\hline \multicolumn{2}{c}{} \\
\end{tabular}


Table 5. Mineralogy in H-4-Continued.

\begin{tabular}{lc}
\hline Sample name: & H4-655 \\
Lithology & $\begin{array}{c}\text { Gray matrix-supported } \\
\text { breccia } \\
\text { QAP }\end{array}$ \\
Alteration & Normalized results \\
Weight $\%$
\end{tabular}

Sample name: $\quad$ H4-690

Lithology Medium gray lavas

Alteration QSP-p

Normalized results

Mineral
NON-CLAYS

Quartz

41.1

Anhydrite

0.3

Anatase

0.1

Rutile

0.6

Pyrite

12.1

Alunite

0.1

Total non-clays

54.3

CLAYS

Illite/Muscovite

41.2

Pyrophyllite

4.6

45.7

FPF

0.0979

100 
Table 5. $\quad 31$

Table 5. Mineralogy in H-4-Continued.

\begin{tabular}{|c|c|c|c|}
\hline & & & \\
\hline Sample name: & $\mathrm{H} 4-711$ & Sample name: & $\mathrm{H} 4-720$ \\
\hline Lithology & Gray breccia & Lithology & $\begin{array}{c}\text { Gray matrix supported } \\
\text { breccia }\end{array}$ \\
\hline Alteration & QPS & Alteration & QAP \\
\hline & Normalized results & & Normalized results \\
\hline Mineral & Weight \% & Mineral & Weight $\%$ \\
\hline NON-CLAYS & & NON-CLAYS & \\
\hline Quartz & 33.6 & Quartz & 44.1 \\
\hline Anhydrite & 0.2 & Anhydrite & 1.1 \\
\hline Gypsum & 0.1 & Gypsum & 0.1 \\
\hline Jarosite & 0.3 & Magnetite & 0.5 \\
\hline Maghemite & 0.3 & Hematite & 0.2 \\
\hline Rutile & 0.7 & Rutile & 0.8 \\
\hline Pyrite & 11.1 & Pyrite & 9.4 \\
\hline Alunite & 0.4 & Alunite & 6.3 \\
\hline Total non-clays & 46.6 & Natroalunite & 11.3 \\
\hline & & Total non-clays & 73.8 \\
\hline CLAYS & & & \\
\hline Illite/Muscovite & 24.4 & CLAYS & \\
\hline Pyrophyllite & 29.0 & Illite/Muscovite & 1.1 \\
\hline Total clays & 53.4 & Pyrophyllite & 25.1 \\
\hline FPF & 0.0993 & Total clays & 26.2 \\
\hline TOTAL & 100 & FPF & 0.9989 \\
\hline & & TOTAL & 100 \\
\hline
\end{tabular}




\section{Mineralogy from Cores in Prospect Gulch, San Juan County, Colorado}

Table 6. Mineralogy in H-5 samples from X-ray diffraction analyses (sample number indicates depth in feet).

[FPF, full pattern fit]

\begin{tabular}{|c|c|c|}
\hline Alteration type & & $\begin{array}{l}\text { Alteration assemblage } \\
\text { as mapped in fig. } 2\end{array}$ \\
\hline $\begin{array}{l}\text { QSP } \\
\text { QAp }\end{array}$ & Quartz-sericite-pyrite & $\begin{array}{l}\text { qsp } \\
\text { qap }\end{array}$ \\
\hline
\end{tabular}

\begin{tabular}{lc}
\hline Sample name: & H5-20 \\
Lithology & Light gray lavas \\
Alteration & QSP \\
\hline \multicolumn{1}{c}{ Mineral } & Normalized results \\
Weight $\%$
\end{tabular}

\begin{tabular}{lc} 
Sample name: & H5-25 \\
Lithology & Light gray lavas \\
Alteration & Normalized results \\
\multicolumn{1}{c}{ Mineral } & Weight \% \\
$\quad$ NON-CLAYS & \\
Quartz & 44.6 \\
Anatase & 0.6 \\
Pyrite & 2.2 \\
$\quad$ Total non-clays & 47.3 \\
$\quad$ CLAYS & \\
Illite/Muscovite & \\
Pyrophyllite & 52.3 \\
$\quad$ Total clays & 0.3 \\
FPF & 52.7 \\
TOTAL & 0.1064 \\
\hline
\end{tabular}


Table 6. 33

Table 6. Mineralogy in $\mathrm{H}-5$ samples_-Continued.

\begin{tabular}{|c|c|c|c|}
\hline & & & \\
\hline Sample name: & $\mathrm{H} 5-30$ & Sample name: & H5-36 \\
\hline Lithology & Gray lavas & Lithology & $\begin{array}{c}\text { Gray matrix-supported } \\
\text { breccia }\end{array}$ \\
\hline Alteration & QSP & Alteration & QSP \\
\hline & Normalized results & & Normalized results \\
\hline Mineral & Weight \% & Mineral & Weight $\%$ \\
\hline NON-CLAYS & & NON-CLAYS & \\
\hline Quartz & 40.0 & Quartz & 27.3 \\
\hline Anhydrite & 0.2 & Anhydrite & 0.0 \\
\hline Gypsum & 0.0 & Gypsum & 0.6 \\
\hline Barite & 0.3 & Jarosite & 0.7 \\
\hline Goethite & 0.4 & Anatase & 0.5 \\
\hline Pyrite & 7.9 & Pyrite & 8.4 \\
\hline Total non-clays & 48.9 & Total non-clays & 37.6 \\
\hline CLAYS & & CLAYS & \\
\hline Illite/Muscovite & 51.1 & Illite/Muscovite & 62.4 \\
\hline Total clays & 51.1 & Total clays & 62.4 \\
\hline FPF & 0.1117 & FPF & 0.1017 \\
\hline TOTAL & 100 & TOTAL & 100 \\
\hline Sample name: & H5-386 & Sample name: & H5-434 \\
\hline Lithology & $\begin{array}{c}\text { Gray matrix- } \\
\text { supported breccia }\end{array}$ & Lithology & $\begin{array}{c}\text { Gray matrix-supported } \\
\text { breccia }\end{array}$ \\
\hline Alteration & QAp & Alteration & QSP \\
\hline & Normalized results & & Normalized results \\
\hline Mineral & Weight $\%$ & Mineral & Weight $\%$ \\
\hline NON-CLAYS & & NON-CLAYS & \\
\hline Quartz & 52.3 & Quartz & 42.0 \\
\hline Anhydrite & 1.8 & Anhydrite & 0.3 \\
\hline Maghemite & 1.5 & Gypsum & 0.0 \\
\hline Anatase & 0.2 & Anatase & 0.8 \\
\hline Rutile & 0.9 & Rutile & 0.0 \\
\hline Pyrite & 3.1 & Pyrite & 2.0 \\
\hline Alunite & 25.7 & Alunite & 0.1 \\
\hline Natroalunite & 10.1 & Total non-clays & 45.2 \\
\hline Total non-clays & 95.4 & & \\
\hline & & CLAYS & \\
\hline CLAYS & & Illite/Muscovite & 53.5 \\
\hline Pyrophyllite & 4.6 & Pyrophyllite & 1.3 \\
\hline Total clays & 4.6 & Total clays & 54.8 \\
\hline FPF & 0.0991 & FPF & 0.1016 \\
\hline TOTAL & 100 & TOTAL & 100 \\
\hline
\end{tabular}


Table 7. Mineralogy in H-6 samples from X-ray diffraction analyses (sample number indicates depth in feet).

[FPF, full pattern fit

\begin{tabular}{lll}
\hline Alteration type & & $\begin{array}{l}\text { Alteration assemblage as } \\
\text { mapped in fig. 2 }\end{array}$ \\
\hline QSP-p & $\begin{array}{l}\text { Quartz-sericite-pyrite with minor } \\
\text { pyrophyllite }\end{array}$ & qsp \\
QSP-P & $\begin{array}{l}\text { Quartz-sericite-pyrite with major } \\
\text { pyrophyllite }\end{array}$ & $\begin{array}{l}\text { qap-s } \\
\text { qap-s }\end{array}$ \\
QPS & $\begin{array}{l}\text { Quartz-pyrophyllite with major sericite } \\
\text { Quartz-pyrophyllite with minor sericite }\end{array}$ & \begin{tabular}{l} 
qap-s \\
\hline
\end{tabular} \\
\hline
\end{tabular}

\begin{tabular}{lc} 
Sample name: & H6-80 \\
Lithology & $\begin{array}{c}\text { Medium gray lavas } \\
\text { QSP-p }\end{array}$ \\
Alteration & Normalized \\
\multicolumn{1}{c}{ Mineral } & Weight \% \\
$\quad$ NON-CLAYS & \\
Quartz & 35.7 \\
Anhydrite & 0.3 \\
Gypsum & 0.6 \\
Jarosite & 0.4 \\
Maghemite & 0.5 \\
Anatase & 0.1 \\
Rutile & 0.2 \\
Pyrite & 10.0 \\
$\quad$ Total non-clays & 47.8 \\
$\quad$ CLAYS & \\
Illite/Muscovite & \\
Pyrophyllite & 49.4 \\
$\quad$ Total clays & 2.8 \\
FPF & 52.2 \\
TOTAL & 0.0969 \\
\hline
\end{tabular}

\begin{tabular}{|c|c|}
\hline Sample name: & $\mathrm{H} 6-87$ \\
\hline $\begin{array}{l}\text { Lithology } \\
\text { Alteration }\end{array}$ & $\begin{array}{c}\text { Dark gray lavas } \\
\text { QSP-p }\end{array}$ \\
\hline $\begin{array}{c}\text { Mineral } \\
\text { NON-CLAYS }\end{array}$ & $\begin{array}{l}\text { Normalized } \\
\text { Weight \% }\end{array}$ \\
\hline Quartz & 30.0 \\
\hline Anhydrite & 0.5 \\
\hline Gypsum & 0.2 \\
\hline Hematite & 0.1 \\
\hline Maghemite & 1.4 \\
\hline Anatase & 0.0 \\
\hline Rutile & 0.4 \\
\hline Pyrite & 10.6 \\
\hline Total non-clays & 43.2 \\
\hline \multicolumn{2}{|l|}{ CLAYS } \\
\hline Illite/Muscovite & 53.4 \\
\hline Pyrophyllite & 3.4 \\
\hline Total clays & 56.8 \\
\hline FPF & 0.1201 \\
\hline TOTAL & 100 \\
\hline
\end{tabular}


Table 7. $\quad 35$

Table 7. Mineralogy in H-6-Continued.

\begin{tabular}{lc}
\hline Sample name: & H6-451 \\
Lithology & Dark gray lavas \\
Alteration & QPS \\
\hline \multicolumn{1}{c}{ Mineral } & Normalized \\
$\quad$ NON-CLAYS & \\
Quartz & 34.1 \\
Anhydrite & 0.3 \\
Gypsum & 0.3 \\
Goethite & 0.3 \\
Rutile & 0.8 \\
Pyrite & 8.1 \\
Bassanite & 0.2 \\
Alunite & 0.6 \\
Jarosite & 0.1 \\
$\quad$ Total non-clays & 44.9 \\
$\quad$ CLAYS & \\
Illite/Muscovite & 24.7 \\
Pyrophyllite & 30.4 \\
$\quad$ Total clays & 55.13 \\
FPF & 0.0967 \\
TOTAL & 100 \\
\hline
\end{tabular}

\begin{tabular}{lc}
\hline Sample name: & H6-458 \\
Lithology & $\begin{array}{c}\text { Dark clast-supported } \\
\text { breccia } \\
\text { QSP-P }\end{array}$ \\
Alteration & Normalized \\
\multicolumn{1}{c}{ Mineral } & Weight \% \\
$\quad$ NON-CLAYS & \\
Quartz & 35.0 \\
Anhydrite & 0.2 \\
Anatase & 0.3 \\
Rutile & 0.4 \\
Pyrite & 10.8 \\
Total non-clays & 46.7 \\
$\quad$ CLAYS & \\
Illite/Muscovite & \\
Pyrophyllite & 38.4 \\
$\quad$ Total clays & 14.8 \\
FPF & 53.3 \\
TOTAL & 0.0985 \\
\hline
\end{tabular}


Table 7. Mineralogy in H-6-Continued.

\begin{tabular}{lc}
\hline Sample name: & H6-460 \\
Lithology & $\begin{array}{c}\text { Dark clast-supported breccia } \\
\text { QPs }\end{array}$ \\
Alteration & Normalized \\
\multicolumn{1}{c}{ Weight $\%$} \\
Mineral & 37.8 \\
NON-CLAYS & 0.1 \\
Quartz & 0.0 \\
Jarosite & 0.6 \\
Anatase & 10.0 \\
Rutile & 0.3 \\
Pyrite & 48.8 \\
Alunite & \\
Total non-clays & \\
& \\
CLAYS & 7.4 \\
Illite/Muscovite & 43.8 \\
Pyrophyllite & 51.2 \\
Total clays & 0.0967 \\
FPF & 100 \\
TOTAL & \\
\hline
\end{tabular}




\section{Appendix A. Core log for UPG-D}

\section{Interval from 22 to $30.6 \mathrm{ft}$. (material above $22 \mathrm{ft}$. was unconsolidated and not cored)}

Interval from 22 to $27 \mathrm{ft}$ comprised of altered dacite porphyry intrusive rock (Tdp) that becomes brecciated near $26.5 \mathrm{ft}$. The dacite porphyry (which could be a large boulder-sized clast in breccia) is cut at high angle by a light gray, finer grained, matrix-supported breccia. The matrix-supported breccia is comprised of clastic material (generally $<1-4 \mathrm{~mm}$ ) but tends to envelop a larger clast of Tdp and dark gray silicified rock (up to $5 \mathrm{~cm}$ ) near its top. The Tdp clast has very irregular and somewhat embayed margins. Although difficult to discern, the breccia could have an intrusive matrix; discerning this from a fine clastic matrix is impossible without thin sections. Both Tdp and breccias are moderately silicified and strongly pyrophyllite-altered. Remnant biotite in Tdp has mostly altered to sericite, however sericite is relatively minor compared to pyrophyllite in entire interval. There are several small faults/fractures with slickensides. A small, high angle fault dipping about $45^{\circ}$ cuts the dacite porphyry near $22.8 \mathrm{ft}$. This small fault zone ( $2 \mathrm{~cm}$ wide) is brecciated and contains angular clasts of altered Tdp that range from several mm's to as much as $2-3 \mathrm{~cm}$. The associated breccia is suspended in a yellow-brown, oxidized clay matrix (fig. 1a). Clay in this fault zone is dominantly dickite or kaolinite with perhaps some pyrophyllite. Two other similar brecciated zones associated with fractures occur at $23.5 \mathrm{ft}(\sim 4 \mathrm{~cm}), 24 \mathrm{ft}(\sim 5 \mathrm{~cm})$, and $25.5 \mathrm{ft}$. Fractures and brecciated fault zones contain two generations of clay (fig. 1b). The youngest clay is yellowbrown and highly oxidized. X-ray diffraction (XRD) studies indicate that these clays contain abundant jarosite, major finegrained muscovite or illite (R3 ordering and $<10 \%$ smectite interlayers)-referred from hereon as sericite-with minor to moderate amounts of dickite. The yellow clay is predated by white clay that commonly fills the same fractures or broken zones (fig. 1b). The later white clay is commonly waxy looking and offset slightly by fractures that contain jarositic, yellow clay. These white clays are comprised of major amounts of dickite, minor to major pyrophyllite (harder varieties may contain more pyrophyllite), and very minor sericite. Dark gray and highly silicified clasts become more abundant at 27 $\mathrm{ft}$. The breccia is still matrix-supported (light gray breccia matrix), however the dark silicified clasts are as much as 50 $\%$ of this breccia. The dark silicified clasts are up to about 5 to $6 \mathrm{~cm}$. Clast margins are commonly rounded but can be very irregular, perhaps owing to alteration by the hydrothermal fluids or fluidized light gray breccias. The silicified clasts also contain moderate amounts of pyrophyllite and minor sericite. A larger brecciated but more coherent 1 foot "clast" of the dark silicified rock shows a high angle but very irregular contact with light gray, matrix-supported breccia at $29 \mathrm{ft}$. The body of this silicified clast is internally shattered and broken (non-rotated breccia), but also becomes net-veined by finer breccia from 29.5 to $30.5 \mathrm{ft}$. White, hard and waxy looking pyrophyllite with some dickite (as described above) fills fractures and formed as a replacement locally adjacent to broken and brecciated fractures. Minor to moderate amounts of fine pyrite tends to be more abundant in the finer grained, matrixsupported breccias. Overall pyrite content in the entire interval ranges from about 3 to $7 \%$. Original pyrite is irregularly oxidized in this core interval, especially adjacent to oxidized fracture/rubble zones (fig 1c). Jarosite and goethite is abundant and commonly localized along this and similar fractures zones. A 1-2 cm light gray, "bleached zone" borders the high angle fracture in figure 1c. Similar bleached zones are present in other core intervals. These bleached zones can easily be mistaken as darker clasts with irregular margins suspended in the light gray, matrix- supported breccia. These textures will be noted in ensuing descriptions as "bleached zone textures" 
Fig. 1a

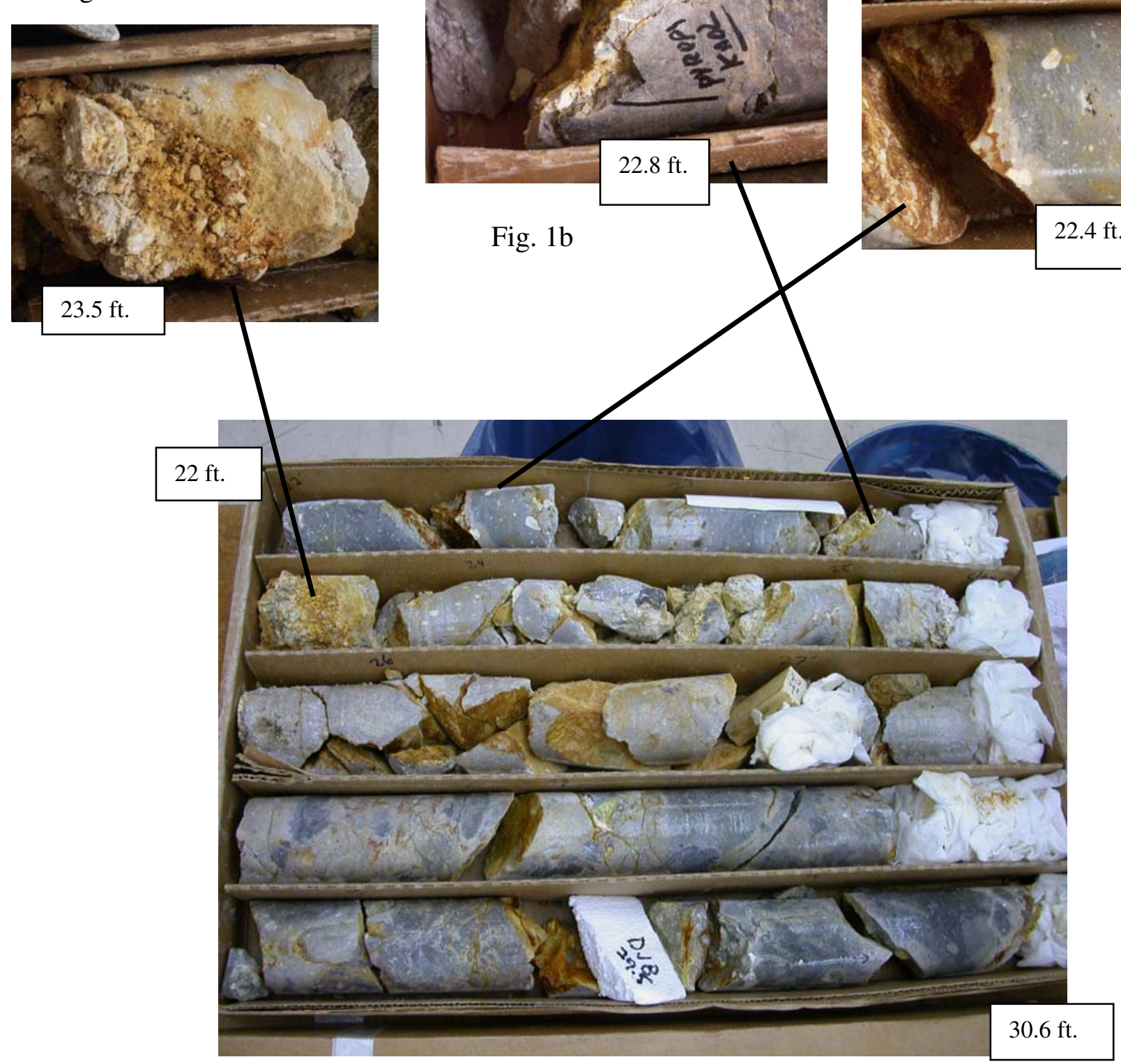




\section{Interval from 30.6 to $38.8 \mathrm{ft}$.}

Breccia continues in this core interval; multiple periods of brecciation make paragenesis quite complex. Matrix-supported breccia continues from 30 to $33 \mathrm{ft}$, however, silicified clasts are less common. Instead, there are a number of clasts of varying lithology with highly reacted margins. "Bleached zone" breccia textures are again localized adjacent to a 5-6 cm, jarosite/goethite oxidized fracture zone near $31 \mathrm{ft}$. These textures may give rise to the dark, irregular and wispy dark margin $(\sim 1 \mathrm{~cm})$ around the light gray, angular clast $(3 \mathrm{~cm})$ at 30.8 ft. Spectral data suggest light and darker zones in "bleached zones" are similarly altered. Similar complex textures and true breccias continue to about 33.5ft. Bleached zones are accentuated around four to five oxidized and clay-lined fractures. The oxidized and clay-lined fracture zones are generally $<0.5 \mathrm{~cm}$ wide with dips from horizontal to high angles; slickensides are present along some of these fractures and appear to postdate some of the jarosite-stained clays. The presence of high temperature clays in these late fractures (dickite and pyrophyllite) and later slickensides, suggest that they formed during later stages of the hydrothermal system. Jarosite and goethite staining may generally reflect later and low temperature acid weathering. At $30.6 \mathrm{ft}$ through $34 \mathrm{ft}$ there are abundant fractures filled with late jarositic clay as described above. Oxidation continues with jarosite and some goethite along with abundant clays along fractures to about $34 \mathrm{ft}$. Below this zone the strong oxidation greatly diminishes. Coarse grained, clastsupported breccia dominates in interval from 33.5 to $35 \mathrm{ft}$. Clasts are angular to subangular $(5-15 \mathrm{~cm})$, dark and silicified (likely altered lavas), and suspended in a light colored, matrixsupported breccia; clasts in the matrix breccia are generally irregular in shape, have subangular to subrounded margins, and are $<1 \mathrm{~cm}$; clasts in the finer matrix are mostly rotated pieces of the dark silicified rock that are healed in a light tan to white, silicified and sericite/pyrophyllite-altered matrix (fig 1d). The percent of larger silicified clasts greatly decreased from 35 to $38.8 \mathrm{ft}$ as the matrix-supported breccia component becomes dominant. However, silicified clasts in the matrixsupported breccia become coarser grained and elongate in places, with subparallel orientations (length 4 to $5 \mathrm{~cm}$ and width about $1 \mathrm{~cm}$ or less; fig. 1e). This entire core interval is mostly unoxidized and contains abundant fine-grained pyrite (averaging 8-10\%).

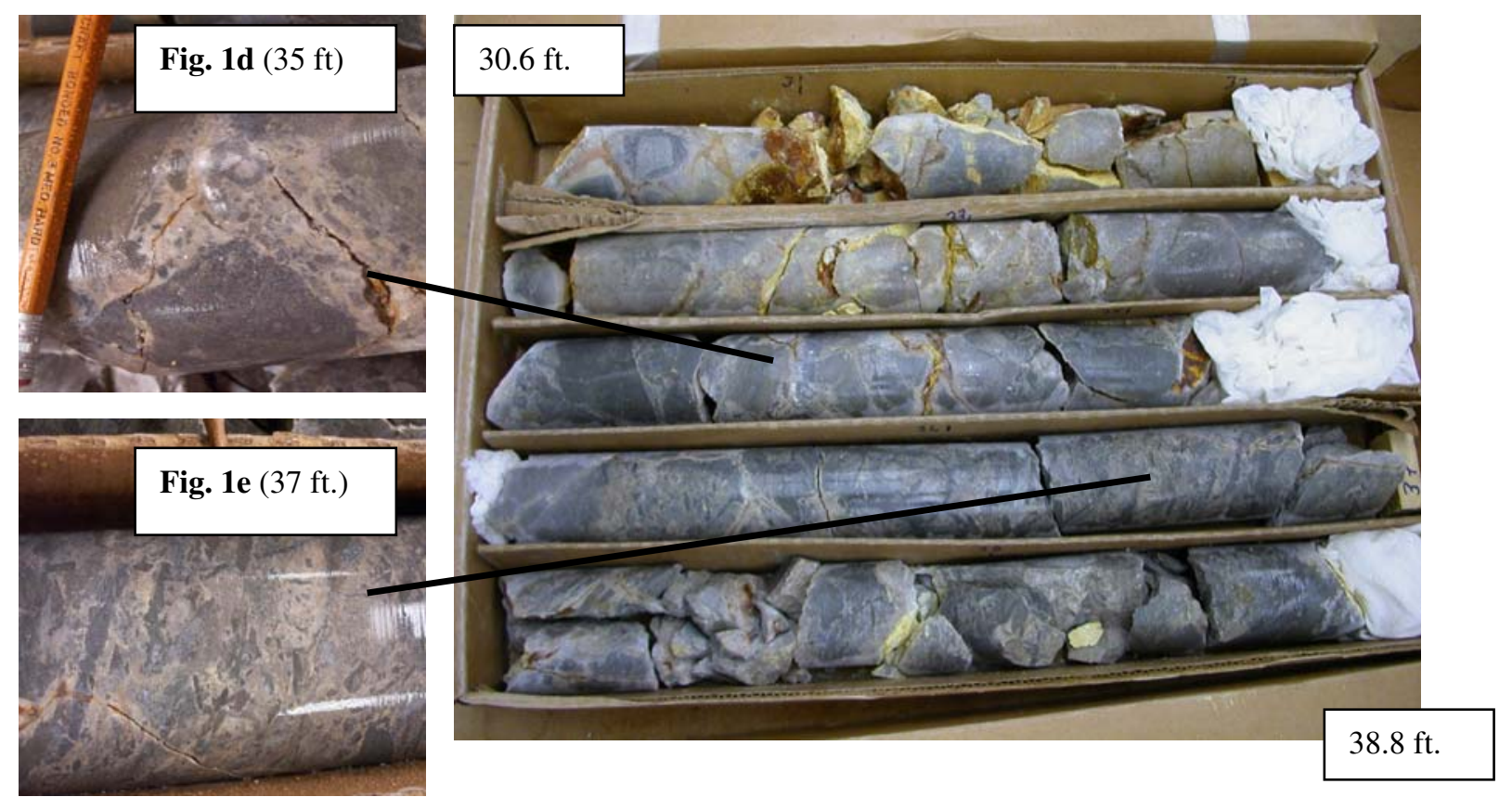




\section{Interval from 38.8 to $47 \mathrm{ft}$.}

These rocks are also hydrothermally brecciated (fine lavas unless otherwise noted) as noted at the bottom of the last core interval; breccia textures are also similar. The clasts are irregular and silicified and commonly elongate. The breccias range from matrix-supported to clast-supported. There are approximately four or five fracture zones with abundant yellow jarosite and associated clays as described above. Rocks in this interval are mildly to moderately silicified with sericite gradually becoming much greater than pyrophyllite in the clay portion; near $47 \mathrm{ft}$, pyrophyllite is absent and sericite dominates these moderately silicified rocks; there are also some fractures filled with white clay consisting mostly of pyrophyllite and lesser dickite. Breccias in interval from about 40 to $47 \mathrm{ft}$. become more clast-supported and clasts are highly elongate and subparallel. Elongate clasts are mostly dark and silicified, and most do not appear to be rotated. Although the matrix is still clastic, bleached zone textures (as described in interval 22 to $30.6 \mathrm{ft}$.) may also be present. Darker clasts are highly silicified with pyrophyllite greater than sericite; alteration in the lighter altered matrix becomes dominated by sericite. There is late, white, and translucent to waxy-looking sericite that cements the breccia clasts at about $58 \mathrm{ft}$. Both clasts and especially breccia matrix contains abundant, finely disseminated pyrite $(7-16 \%)$. Pyrite abundances are similar to that in the intervals above, with jarosite and goethite largely confined to the few local, clay-filled fracture zones.

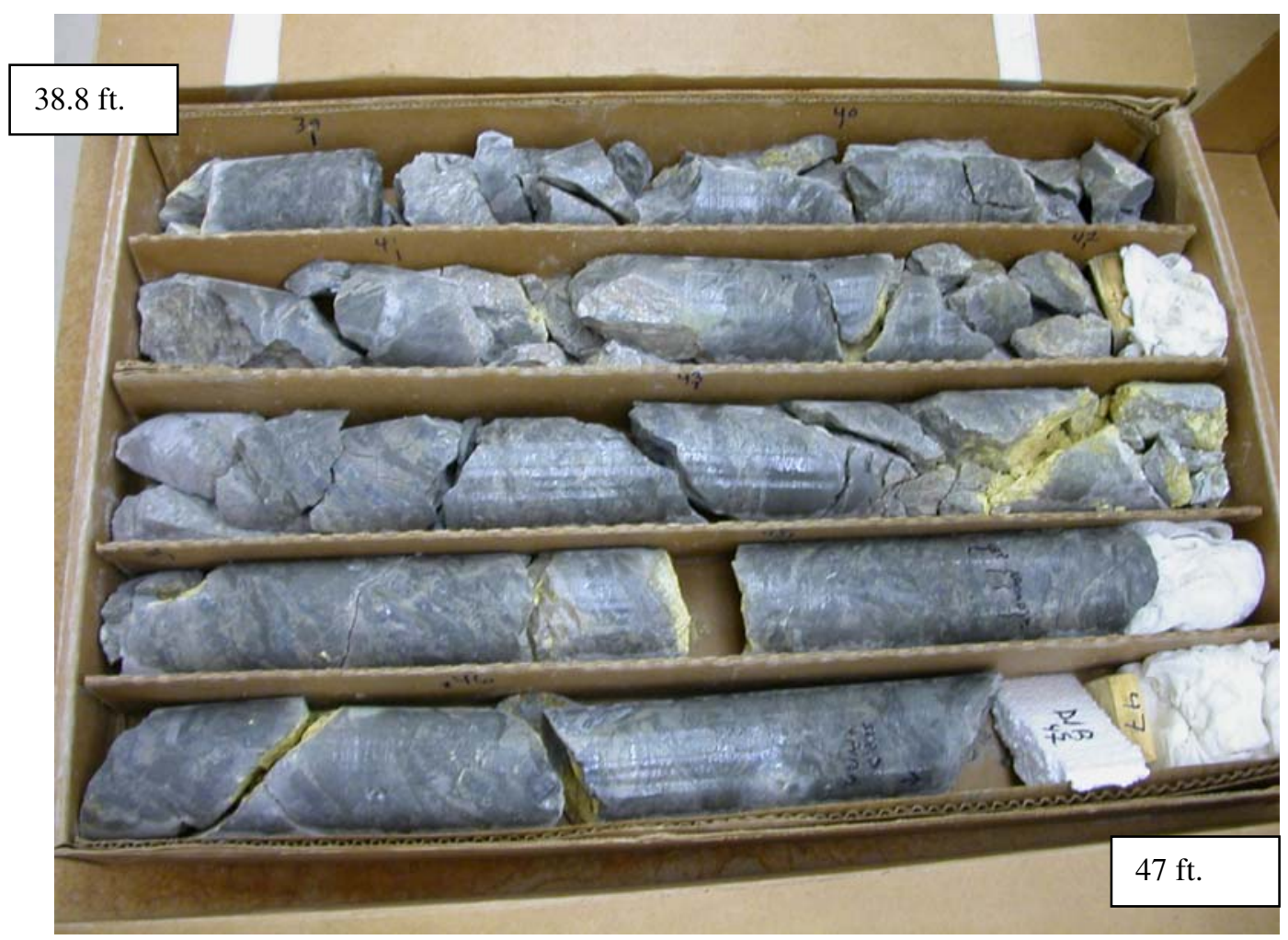




\section{Interval from 47 to $58 \mathrm{ft}$.}

Similarly complex breccias to that described in above intervals. Subparallel clast textures as above with some more equant silicified clasts to $52 \mathrm{ft}$. Lighter gray, matrix-supported and finer grained breccias dominate from 52 to about $54.5 \mathrm{ft}$. Back into coarser grained breccias that are largely clast-supported in interval from 54.5 to $58 \mathrm{ft}$. Clasts in this interval are largely bimodal: dark and silicified as above, and with relic porphyry texture.
Both clast types are as much as $4-5 \mathrm{~cm}$ and have angular to irregular and embayed margins. Some of the porphyritic and altered clasts have white waxy sericite in fractures; these white fractures or clay healed zones are truncated at clast margins. The lighter, finer grained breccia and porphyritic clasts are moderately silicified and have sericite and minor pyrophyllite. Finegrained pyrite is unoxidized in this interval, averaging about $10 \%$. One moderately dipping, oxidized clay-lined fracture cuts the finer matrix-supported breccia at $53 \mathrm{ft}$.

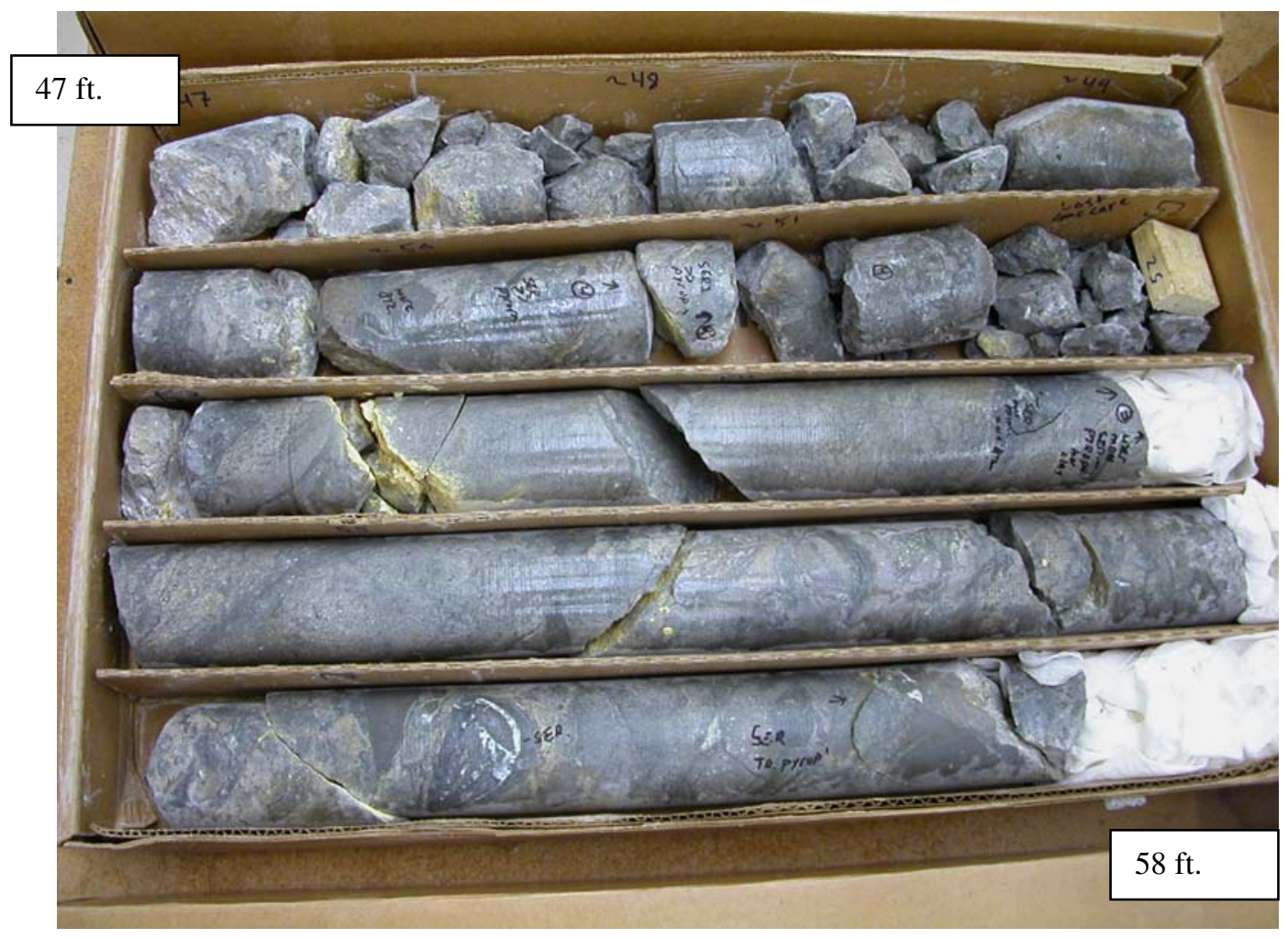




\section{Interval from 58 to $67 \mathrm{ft}$.}

Rocks are dark, highly brecciated and highly silicified; clays are comprised of sericite and minor pyrophyllite. There are several 1 to $3 \mathrm{~mm}$-wide fractures filled with the white soft clay. The white clay consists of dickite with minor to moderate pyrophyllite. The late, yellow-brown, jarositic clay fills some of the same fractures, but post-dates the white clays. Clay XRD studies verify similar compositions to the jarositic clays described above (major R3 sericite; minor to moderate dickite, and abundant jarosite). There are approximately two jarositic and clay-lined fractures in interval to from 58 to $64 \mathrm{ft}$.; a steeply dipping fracture at $64 \mathrm{ft}$. is jarositic and clay lined and has a slickensided slip face. A breccia and rubble zone with abundant jarositic clay continues from the footwall of the fault to $66.7 \mathrm{ft}$. Jarositic clay is abundant throughout the rubble, clasts, and matrix of this footwall interval. The earlier formed, white dickite/pyrophyllite-rich clay is also abundant in this interval. Clasts in the breccia/rubble zone are 1 to $3 \mathrm{~cm}$ and of the same alteration type and lithology as that on the hanging wall of the small fault. Although pyrite is highly oxidized in the brecciated rubble from about 54 to $67 \mathrm{ft}$., it is unoxidized and abundant from 58 to $64 \mathrm{ft}$.

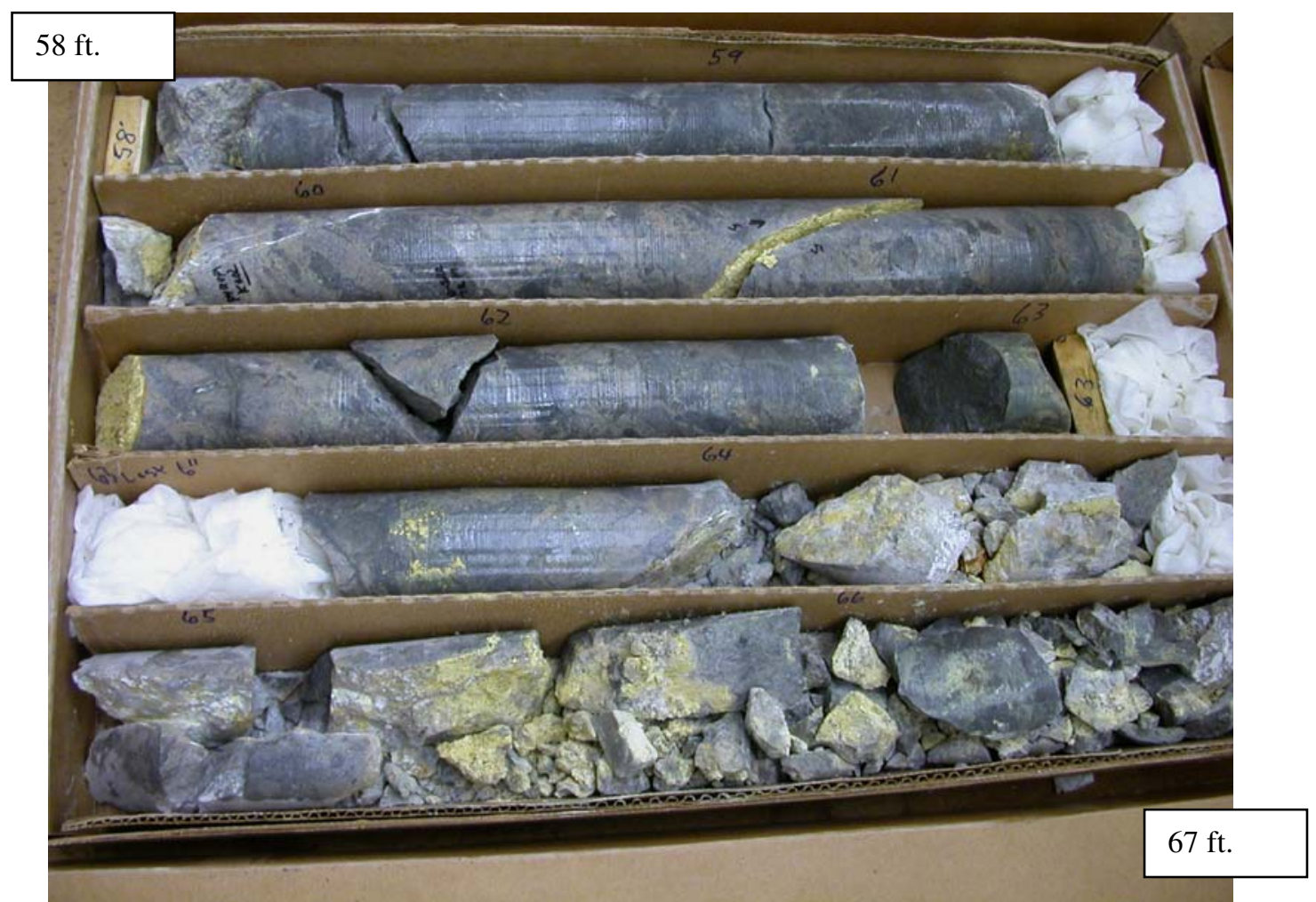




\section{Interval from 67 to $75 \mathrm{ft}$.}

Highly silicified and very dark breccias (fine lavas) from 67 to $68 \mathrm{ft}$.; these rocks are broken and highly fractured in this interval; this zone reflects the margins of the interval described from 64 to $66.7 \mathrm{ft}$. Jarositic clay also is present along fractures from 67 to $68 \mathrm{ft}$. From 68 to $72 \mathrm{ft}$. the rock is dominated by highly silicified and sericitic altered lavas; primary texture is nearly obliterated. Dark gray, massive silicification diminishes from 69 to $72 \mathrm{ft}$. Relic texture of sericitic altered lavas (light gray zones) becomes more recognizable in this 3-ft. interval. The lighter colored, sericitic altered lavas in this zone are cut by abundant stockwork-like fine quartz \pm pyrite veinlets. Rather than a chaotic stockwork, these dense veinlets tend to be more subparallel and have moderate dips. The rocks become very dark and highly silicified from 72 to $75 \mathrm{ft}$. and appear to be highly brecciated and mostly clast-supported. However, the breccia texture in this silicified interval is more difficult to discern. There are some small vugs that average about $0.5 \mathrm{~cm}$ in this last silicified interval. Fine-grained pyrite averages more than $8-10 \%$ in this entire core interval.

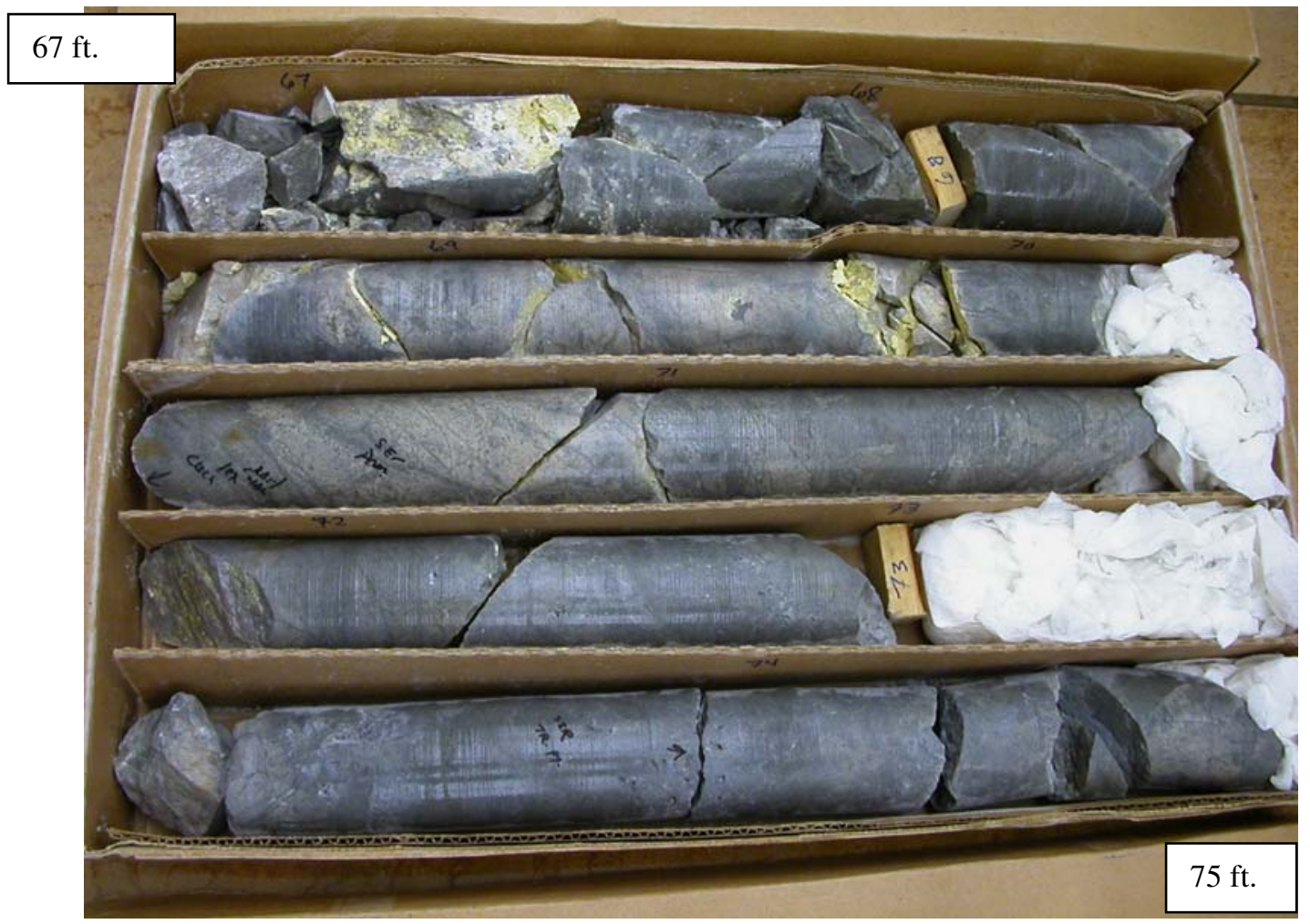




\section{Interval from 75.8 to $83.5 \mathrm{ft}$.}

Interval is similar to above; however, this zone is all highly brecciated and contains zones of very coarse, clast-supported breccia. Some of the breccia clasts are 15 to $20 \mathrm{~cm}$ across and are subrounded to subangular. However, there is some finer breccia that is largely matrix-supported. There are four or five strongly fractured and broken zones in the entire core interval. All of these fractures and broken zones localized the jarositic clay. The nature of breccia and alteration in this interval is quite similar to that described from 38.8 to $47 \mathrm{ft}$. The breccia clasts are dark and highly silicified; sericite is the dominant clay in the lighter matrix dominated portions. Pyrite, as noted above, is finely disseminated and highly abundant, probably on the order $10 \%$ or more. There are also some pyritic veins that cut throughout this entire interval. There are several zones with fractures that contain the white dickite/pyrophyllite clay as described above. These clays are late paragenetically, but pre-date the yellowbrown jarositic clays. The relative paragenesis of these late clays remains consistent with that observed in previous core intervals.

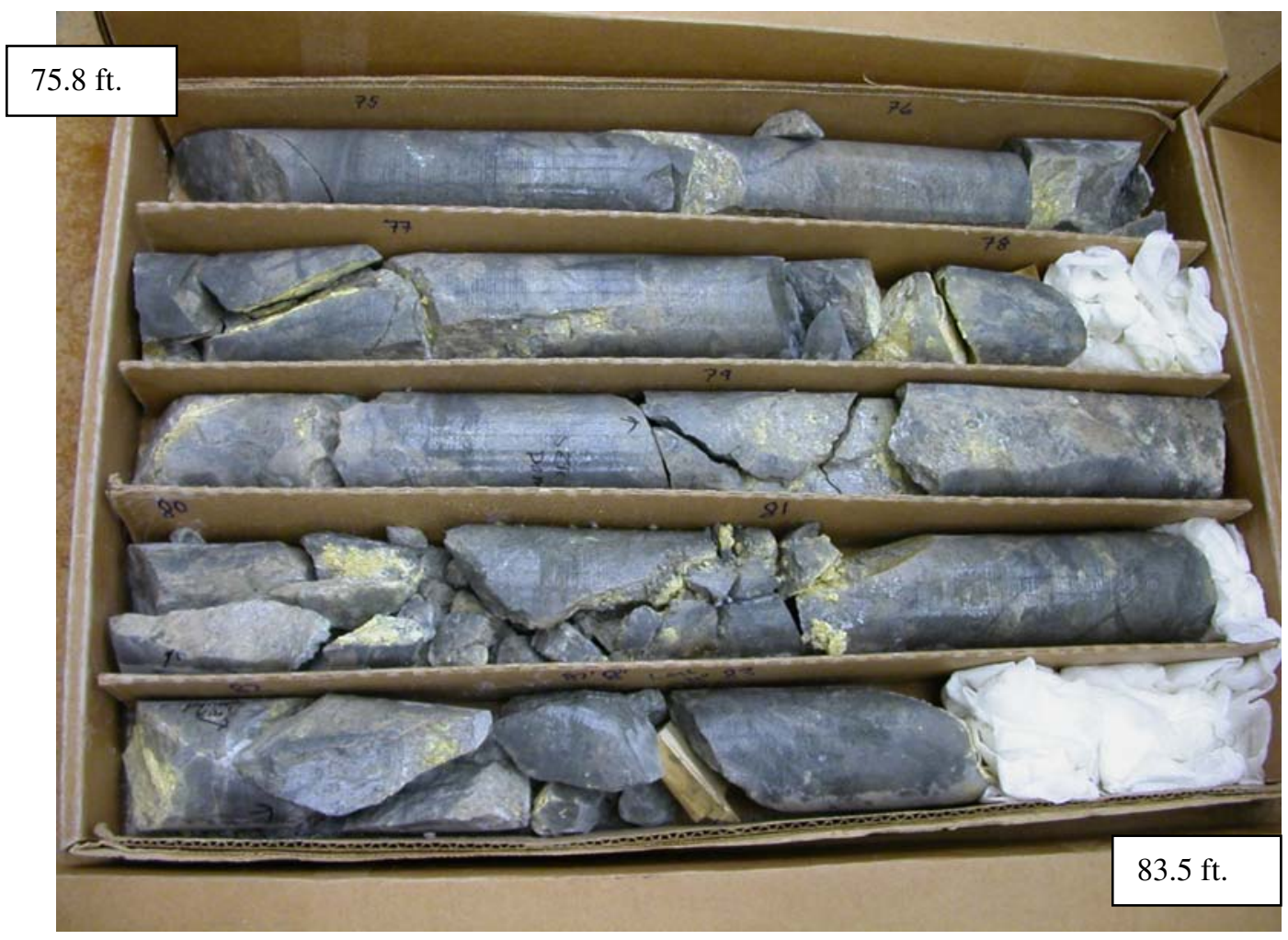




\section{Interval from 83.5 to $91 \mathrm{ft}$.}

There is a high angle, brecciated fracture zone at $83 \mathrm{ft}$. with the late jarositic clay. Rocks are mostly brecciated in this entire interval, however, the breccia textures are more difficult to discern and probably fine grained. The breccia clasts tend to have irregular margins; breccia dominates to about $89 \mathrm{ft}$. Light gray, sericitic altered lavas and likely some breccias are present form 89 to $91 \mathrm{ft}$. These rocks are cut by abundant, very dark, stockwork quartz \pm pyrite veinlets. The margins of the veinlets are rather diffuse with widths ranging for 1-2 mm up to $0.5 \mathrm{~cm}$. Although these still approximate "stockwork" fabric, many veinlets are roughly subparalell with moderate dips. The dips of these and other veinlets throughout this core may be influenced by foliation. With exception of the darker more silicified zones and clasts, lighter colored rocks in this interval are moderately silicified and are dominated by sericitic clays with a trace of pyrophyllite; towards the bottom of the interval pyrophyllite increases. Fine pyrite is abundant in this interval but decreases to about $2-4 \%$ in the last 2 to $3 \mathrm{ft}$.

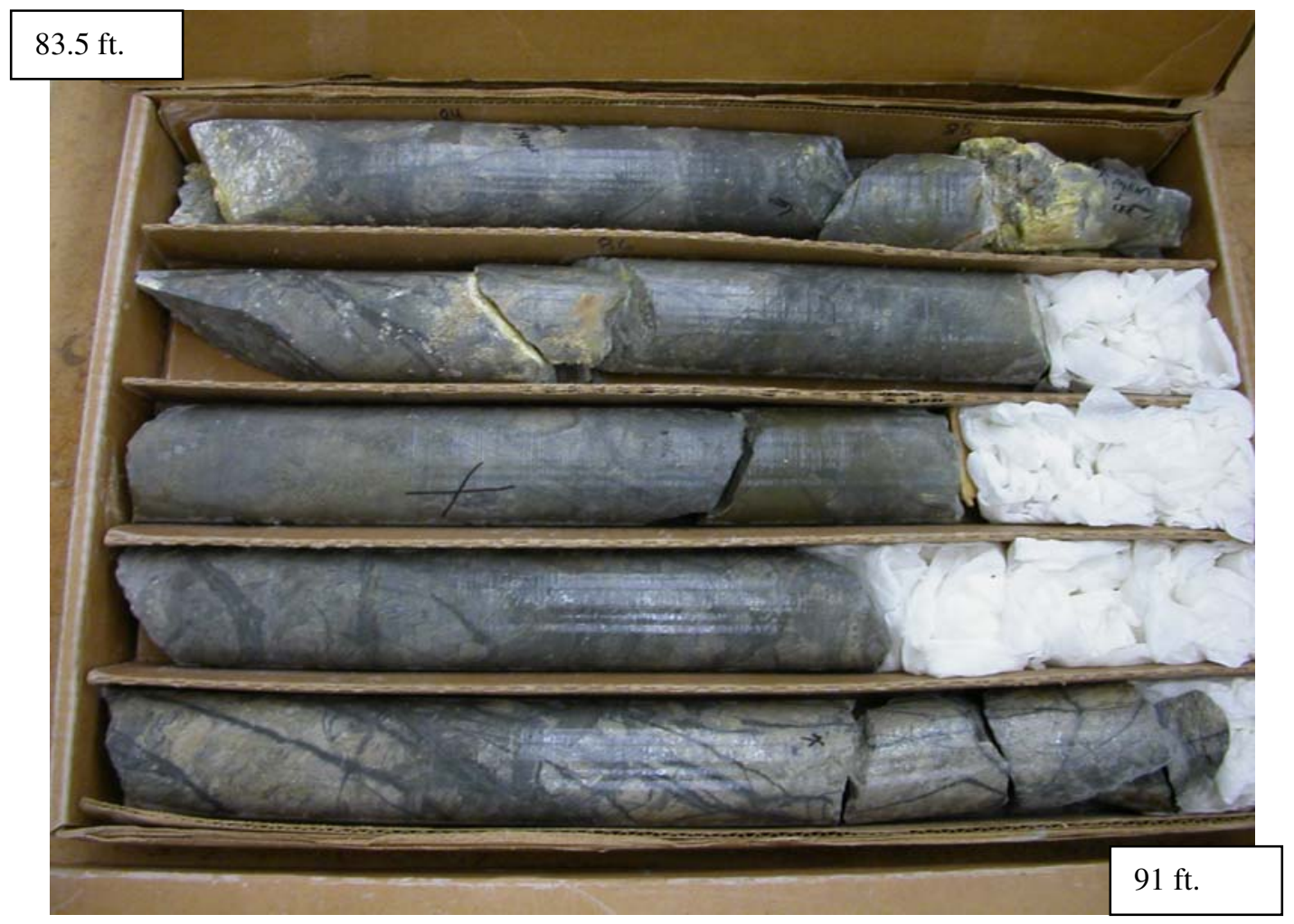




\section{Interval from 91 to $98.5 \mathrm{ft}$.}

Highly brecciated lavas continue; these are cut by abundant, very dark, stockwork quartz \pm pyrite veinlets (see description from 89-91 ft.). The rocks become darker and massively silicified from 95-96 ft. Dark quartz veinlets with diffuse margins emanate from the silicified zone at $95 \mathrm{ft}$. and cut the lighter gray, more sericitic altered, brecciated lavas. Highly broken and probably small fault zones are present at 95, 96 and $97 \mathrm{ft}$. These zones contain late jarositic clay mixed with minor clasts. The late, white dickite/pyrophyllite clay is also present in these zones. Although this interval is highly brecciated, much of the breccia texture is difficult to discern. Except in the dark and highly silicified zones, sericite is much more abundant than pyrophyllite. Pyrophyllite increases toward the bottom of this interval. Pyrite is abundant, as described above.

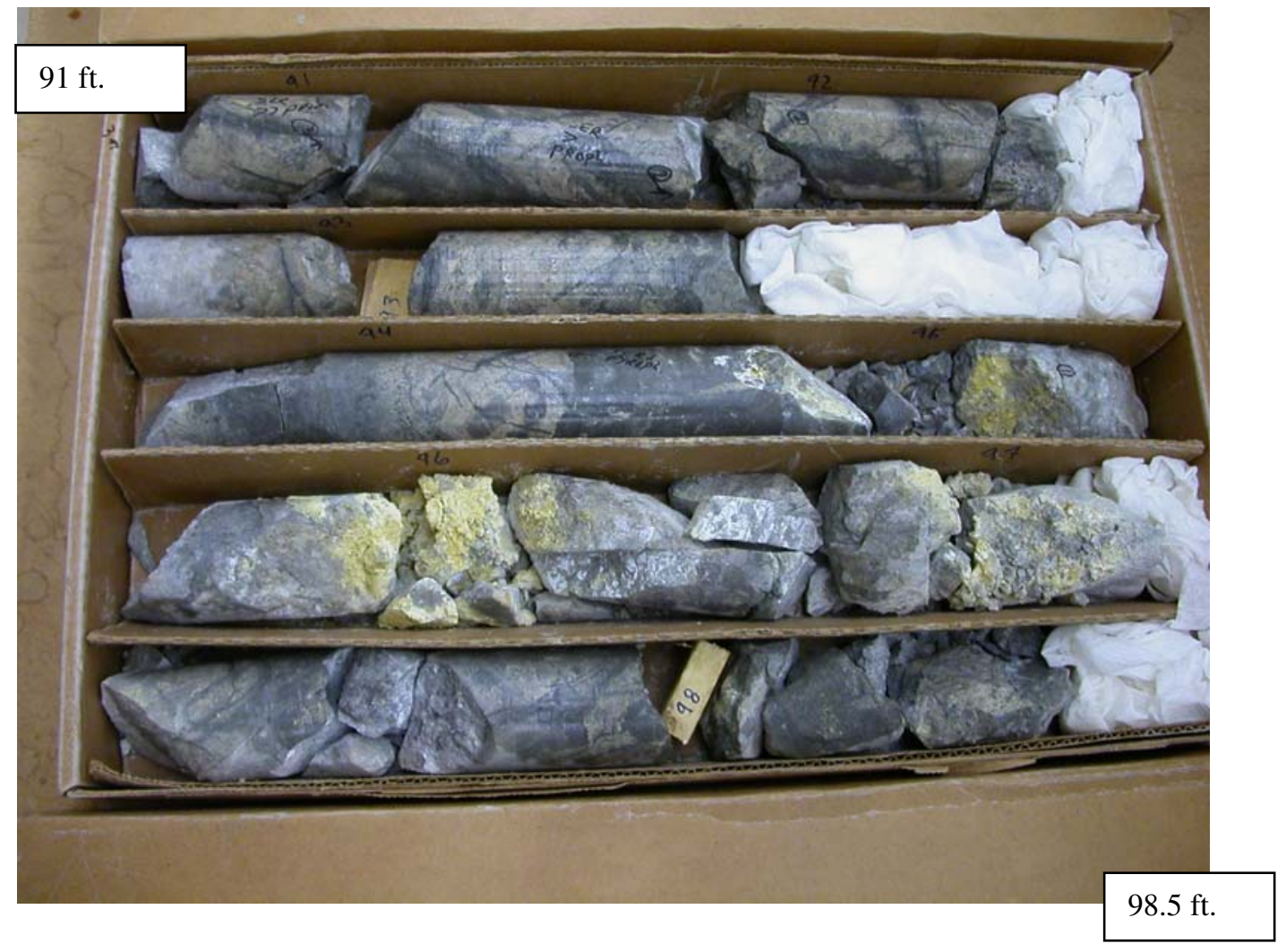




\section{Interval from 98.5 to $106 \mathrm{ft}$.}

Breccia as described above from 98.5 to $99 \mathrm{ft}$. Interval then grades into brecciated dacite porphyry (Tdp) at about $100 \mathrm{ft}$.; breccias alternate with Tdp to $101 \mathrm{ft}$. Dacite porphyry is dominant from 101 to about $106 \mathrm{ft}$. The feldspar phenocrysts in the altered dacite porphyry are white, sericite and pyrophyllitealtered; whereas, the aphanitic groundmass is predominantly quartz-pyrophyllite-altered; pyrophyllite is more abundant than sericite in the dacite groundmass. The dacite porphyry is net veined by breccia or fine-grained breccia zones (described below). There are several fracture zones within this interval; fracture at $100 \mathrm{ft}$. is high angle and contains jarositic clay. Another high angle fracture at $103 \mathrm{ft}$. contains white, waxylooking dickite and pyrophyllite. As described above, these fracture filling clays are paragenetically late; jarositic staining is minimal. A high angle, jarosite-lined fracture is also present at $105 \mathrm{ft}$. This fracture contains some pyrophyllite-kaolinite; these clays are coated by the later jarosite. Most of core interval, with exception of brecciated Tdp zones, is moderately to highly silicified. The more silicified zones are finely vuggy in places. Dacite porphyry zones contain less disseminated pyrite than breccias, but still average roughly $5 \%$ or more. Interval from 101 to $106 \mathrm{ft}$. is characterized by zones with irregular, almost stockwork-like pyrite veinlets; veinlet margins are very diffuse and irregular. Most of these veinlets measure up to 4 to $5 \mathrm{~mm}$ but some are as much as $1 \mathrm{~cm}$ across. Breccia within this interval is mostly matrix-supported to but ranges to clast supported; breccia clasts are commonly subrounded generally less than $1 \mathrm{~cm}$ across.

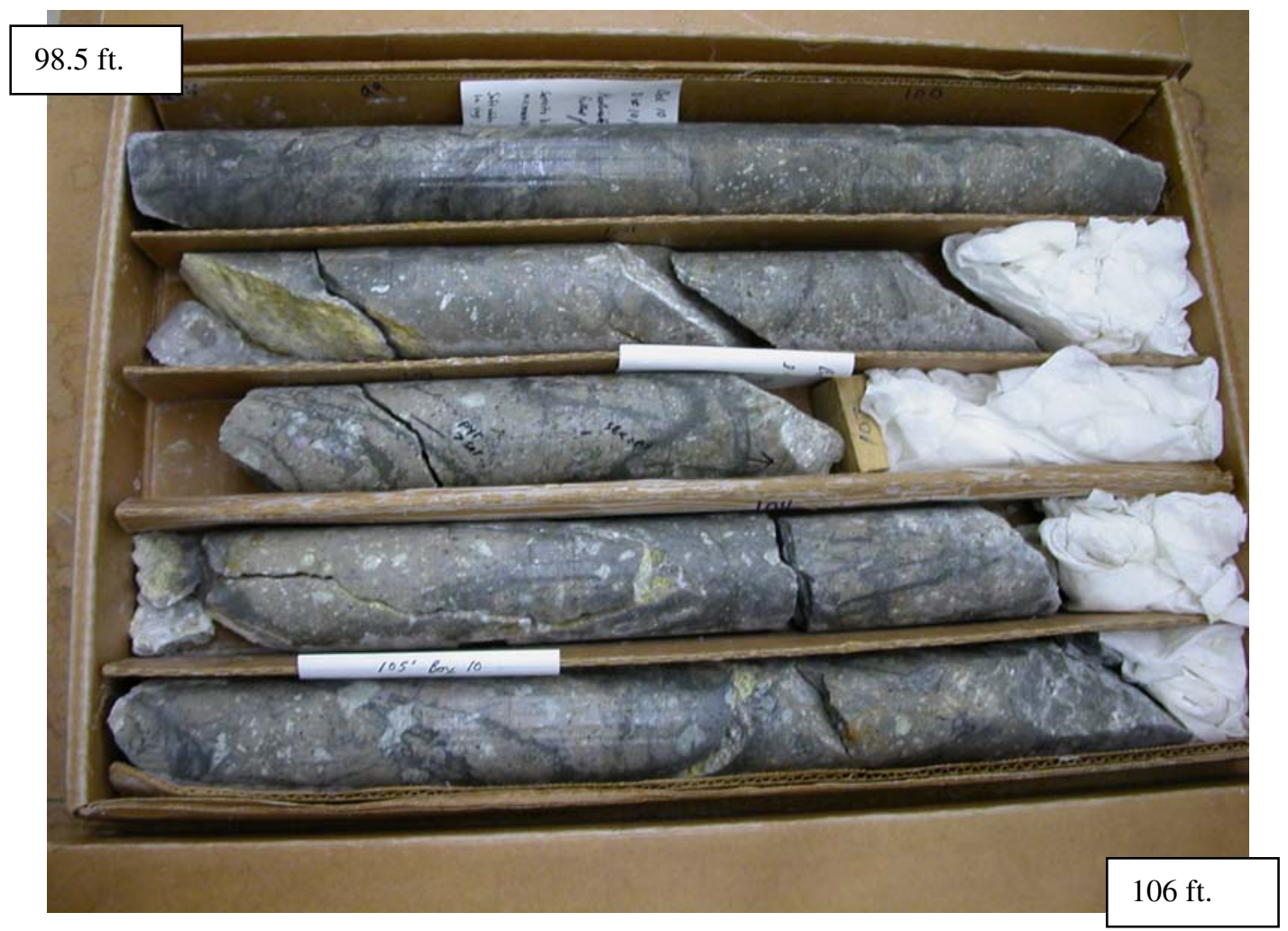




\section{Interval from 106 to $114.5 \mathrm{ft}$.}

The Tdp intrusion (or block of intrusive dacite net veined by breccia) continues to about $110 \mathrm{ft}$. The bottom of Tdp interval has a gradational contact with breccia that coarsens away form the contact. Breccia in interval below has subangular clasts ranging from 15 to $30 \mathrm{~cm}$ across that are in turn suspended in a matrix-supported breccia. There are several high angle fracture zones that contain kaolinite and pyrophyllite; the rest of the interval is highly silicified with abundant disseminated pyrite. Sericite is generally more abundant than pyrophyllite in the more silicified intervals. Alteration of Tdp is similar to that described in the previous interval.

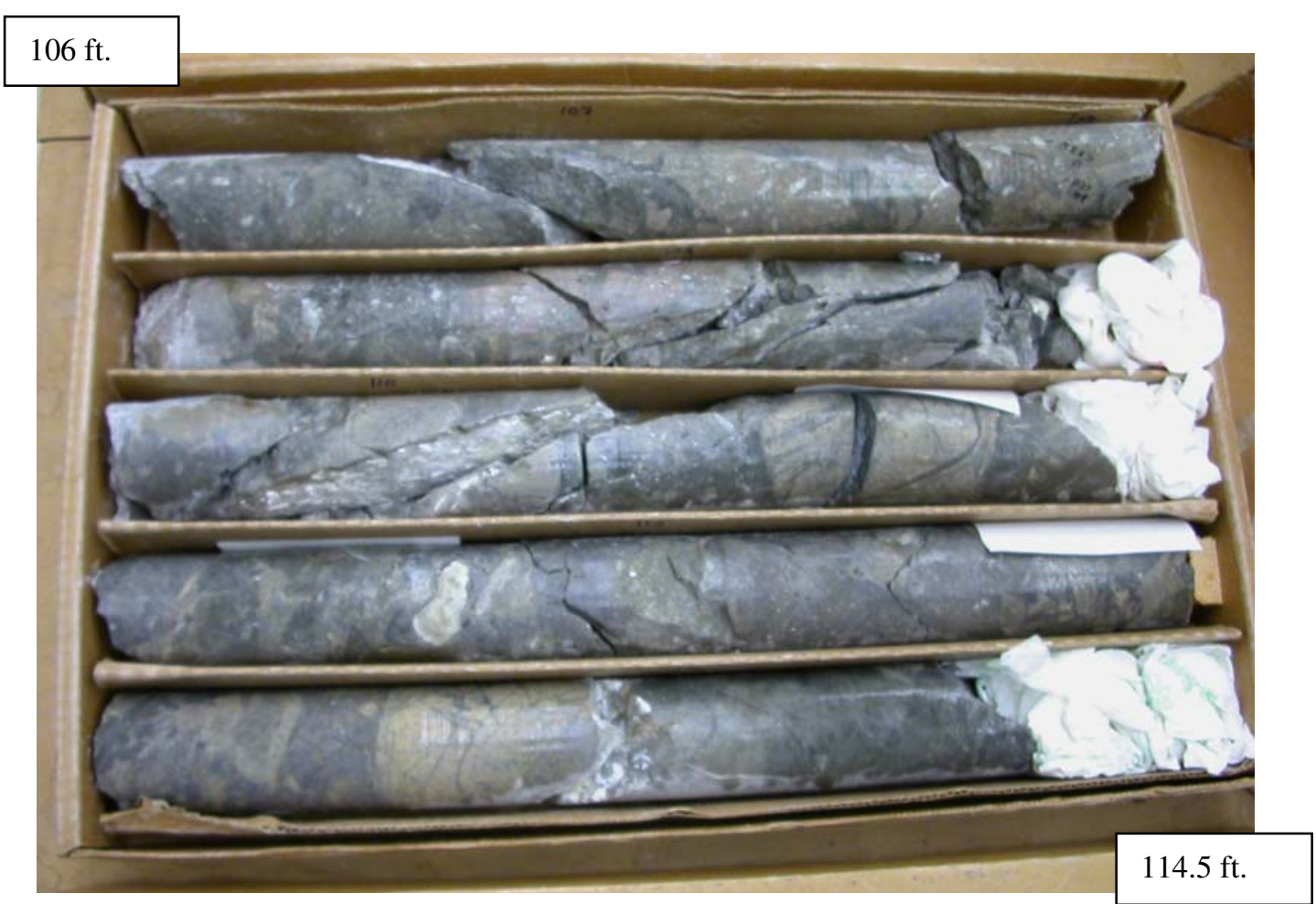




\section{Interval from 114.5 to $123 \mathrm{ft}$.}

This zone is largely coarse-grained, clast-supported breccias that alternate with more matrix-supported hydrothermal breccias. The clasts are highly silicified as observed in above breccia intervals. Breccias are also moderately to highly silicified with sericite much more abundant than pyrophyllite in the clay fraction. Late pyrophyllite and kaolinite are present along a number of fracture zones in this interval. Later jarosite lines these fractures as well.

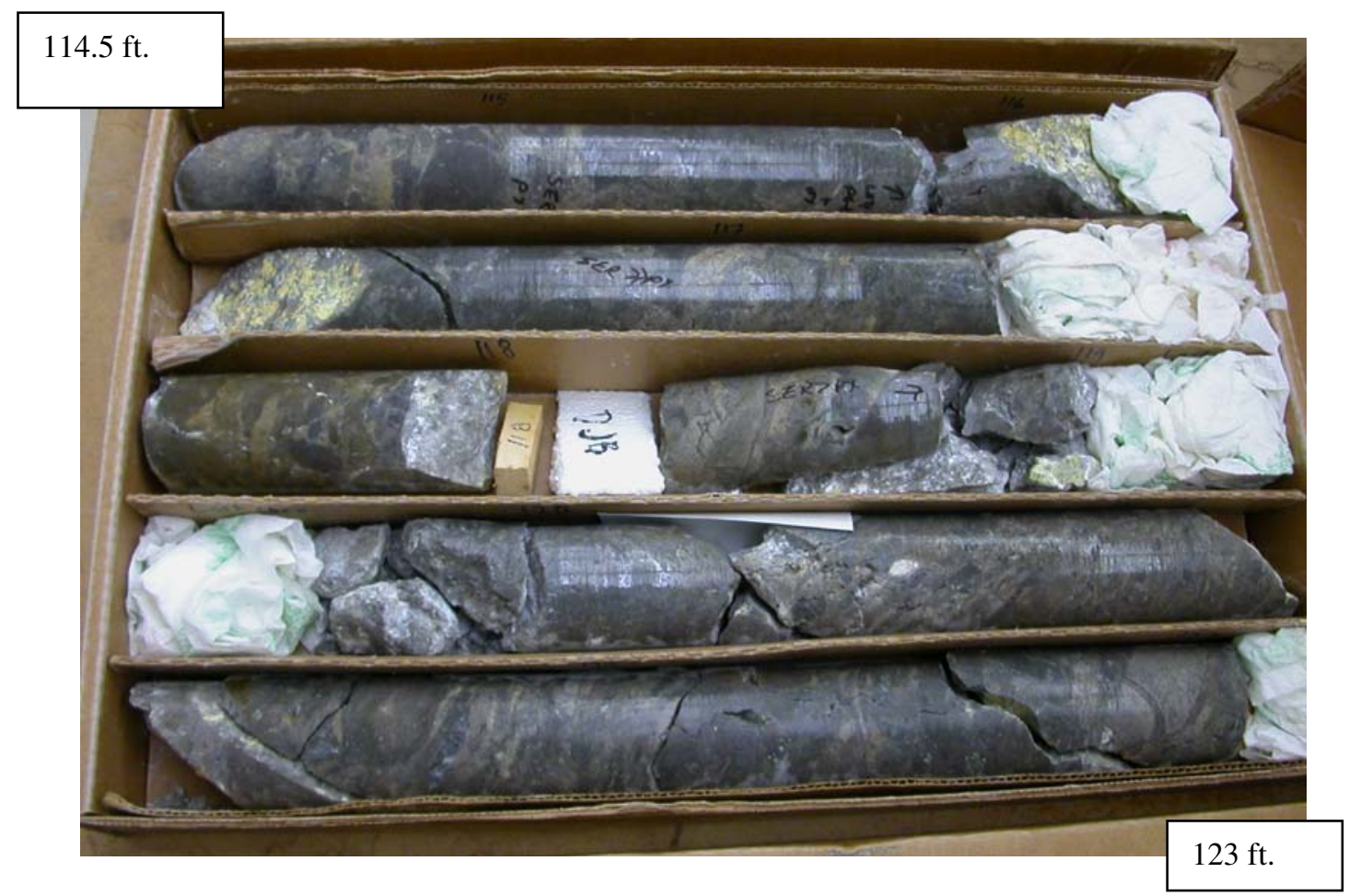




\section{Interval from 123 to $131.5 \mathrm{ft}$.}

Interval is highly brecciated with textures similar to above. Breccias are highly silicified with sericite much more abundant than pyrophyllite to about $125 \mathrm{ft}$. However, at $126 \mathrm{ft}$., sericite and pyrophyllite are approximately subequal. There is a substantial high angle fracture at $128 \mathrm{ft}$. that is lined with thin kaolinite and pyrophyllite and a later thin jarositic clay. Another similar fracture zone is present at about $129 \mathrm{ft}$. There is also abundant vug-filling pyrite that fills the matrix around breccia clasts. The pyrite that fills these smaller and irregular matrix vugs in the matrix is euhedral but still relatively fine-grained.

$123 \mathrm{ft}$.

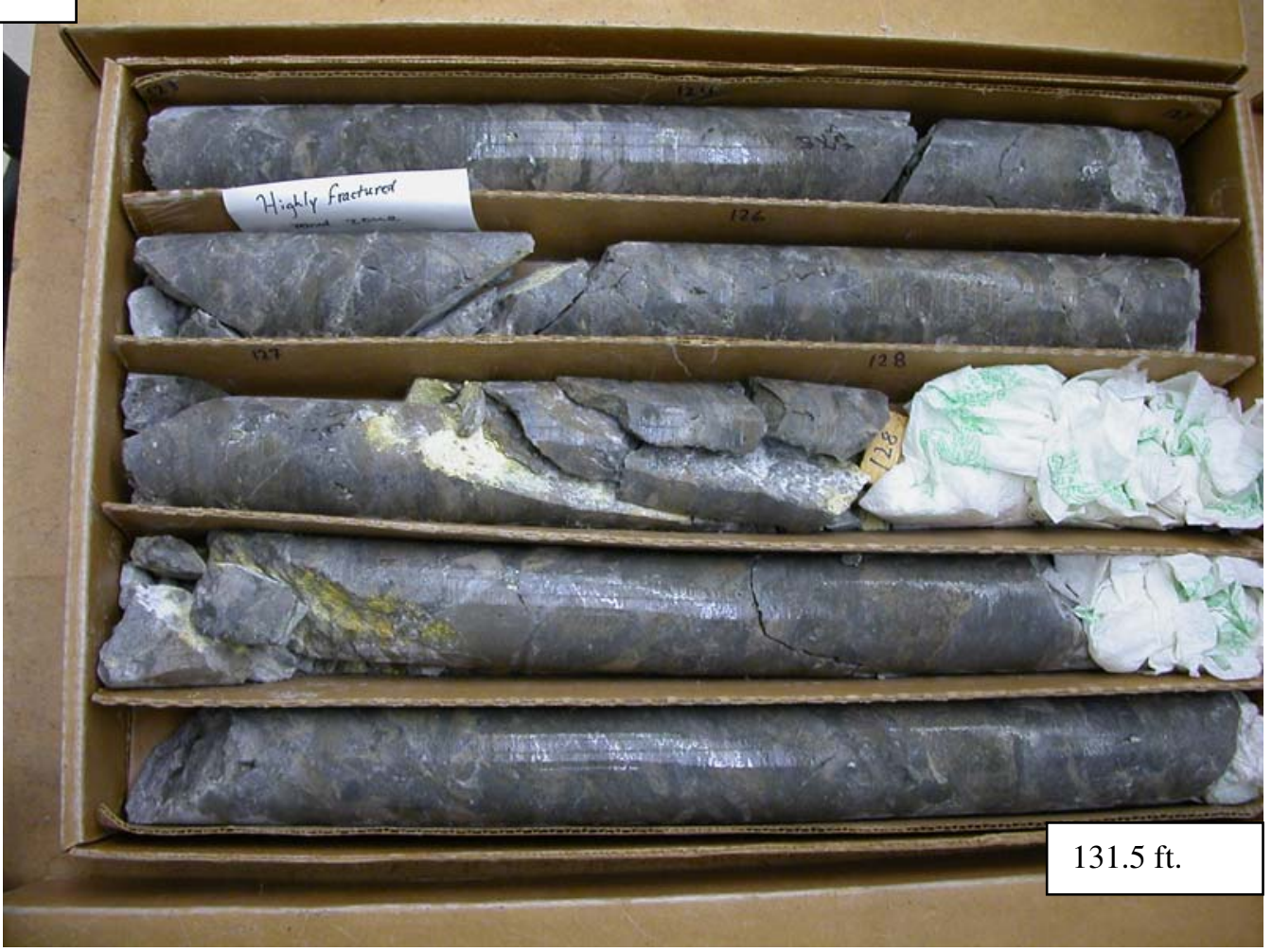




\section{Interval from 131.5 to $138 \mathrm{ft}$.}

Interval contains highly silicified, coarse-grained, clast-supported hydrothermal breccias. Clasts are generally angular to subrounded. Most of the clasts are highly silicified. Pyrophyllite abundance in this silicified interval increases to about $135 \mathrm{ft}$. and is more abundant than sericite. There is a high angle fracturefilling veinlet that contains relatively fine to medium grained, potassium alunite and lesser pyrophyllite at $134 \mathrm{ft}$. At $135 \mathrm{ft}$., pyrophyllite and lesser alunite are present as the major alteration phases-as always less than quartz. Some of the breccias have a matrix healed with alunite and pyrophyllite. The alunite abundance is probably greater than pyrophyllite in these healed breccia zones. There are also some minor vuggy zones that contain late pyrite in some of these breccias; there is also some fine-grained but euhedral pyrite that appears to post-date the alunite veinlets.

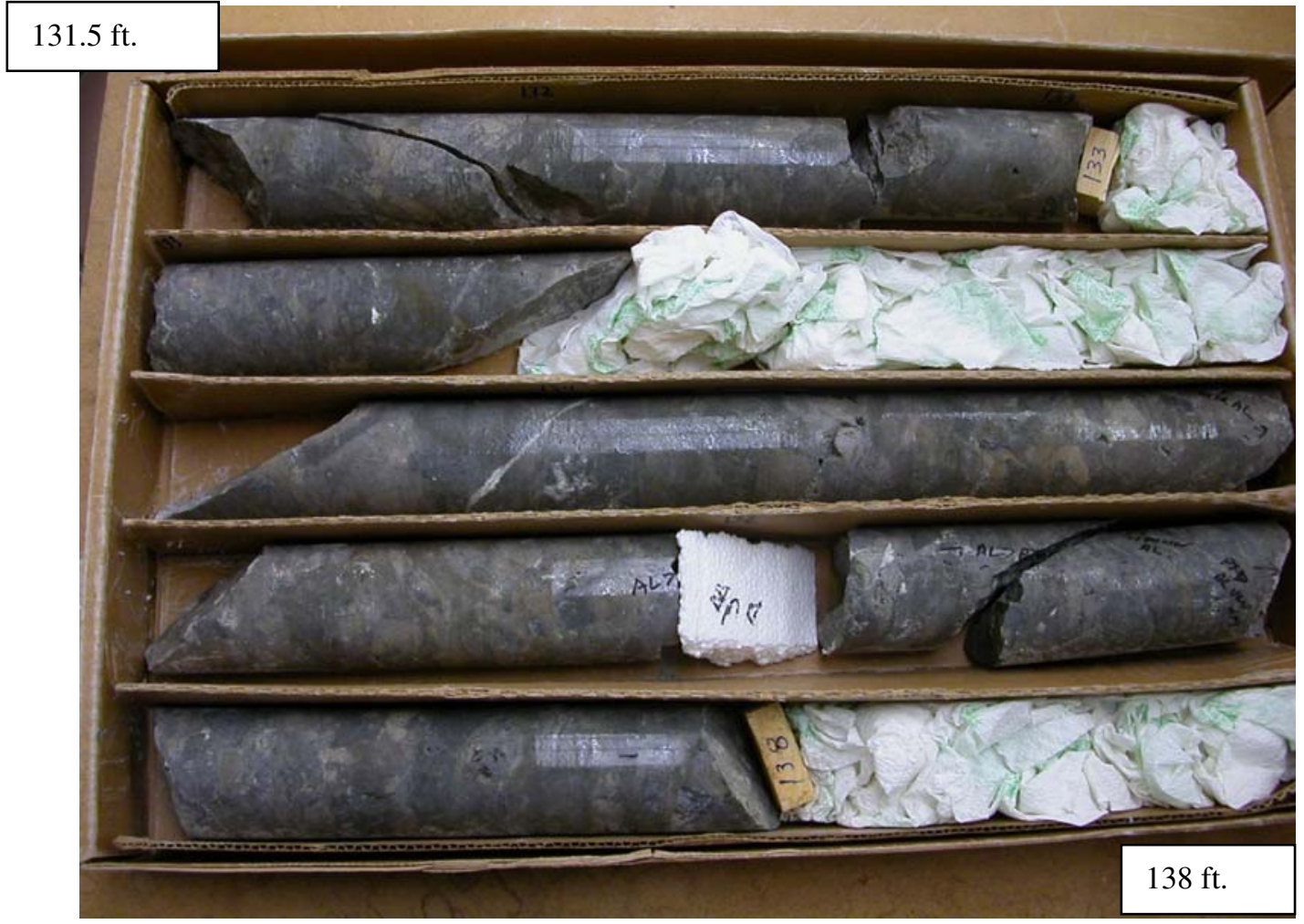

\section{Interval from 138 to $153 \mathrm{ft}$.}

Complex and multi-generational hydrothermal breccias. Rocks are highly silicified and clays consist largely of pyrophyllite; alunite is minor. White fracture-filling pyrophyllite is common. The breccias are mostly clast-supported with subangular to angular clasts; the larger clasts are up to about $15 \mathrm{~cm}$ across. Clasts are comprised of multiple lithologies with different degrees of silicification. There are some later, mostly matrix-supported hydrothermal breccias that cut the coarser grained breccias; these later breccias generally have finer grained clasts. Pyrite throughout this interval is relatively fine-grained and commonly cements the breccia matrix. There are many voids and vuggy areas between clasts. The vuggy areas are partially filled with finer grained pyrite. There are also late pyritic veinlets. These veinlets are about 3-4 mm and cross-cut breccia clasts and matrix. The lithologies and textures of the clasts are difficult to distinguish; however they are likely to be fine-grained lavas (Tsv). Breccias become clast-supported in the interval from 151 to $153 \mathrm{ft}$. The clasts appear to be mostly non-rotated and comprised largely of fine-grained lavas of the Silverton Volcanics (Tsv); some of the altered feldspar phenocrysts are recognizable in the clasts. Clasts in this interval range from $10-15 \mathrm{~cm}$ across. A fracture at $149 \mathrm{ft}$. contains the yellow oxidized clay that is predominantly jarositic with abundant sericite and minor kaolinite/dickite. There is another low angle fracture at $157 \mathrm{ft}$. with the same yellow clay assemblage. 

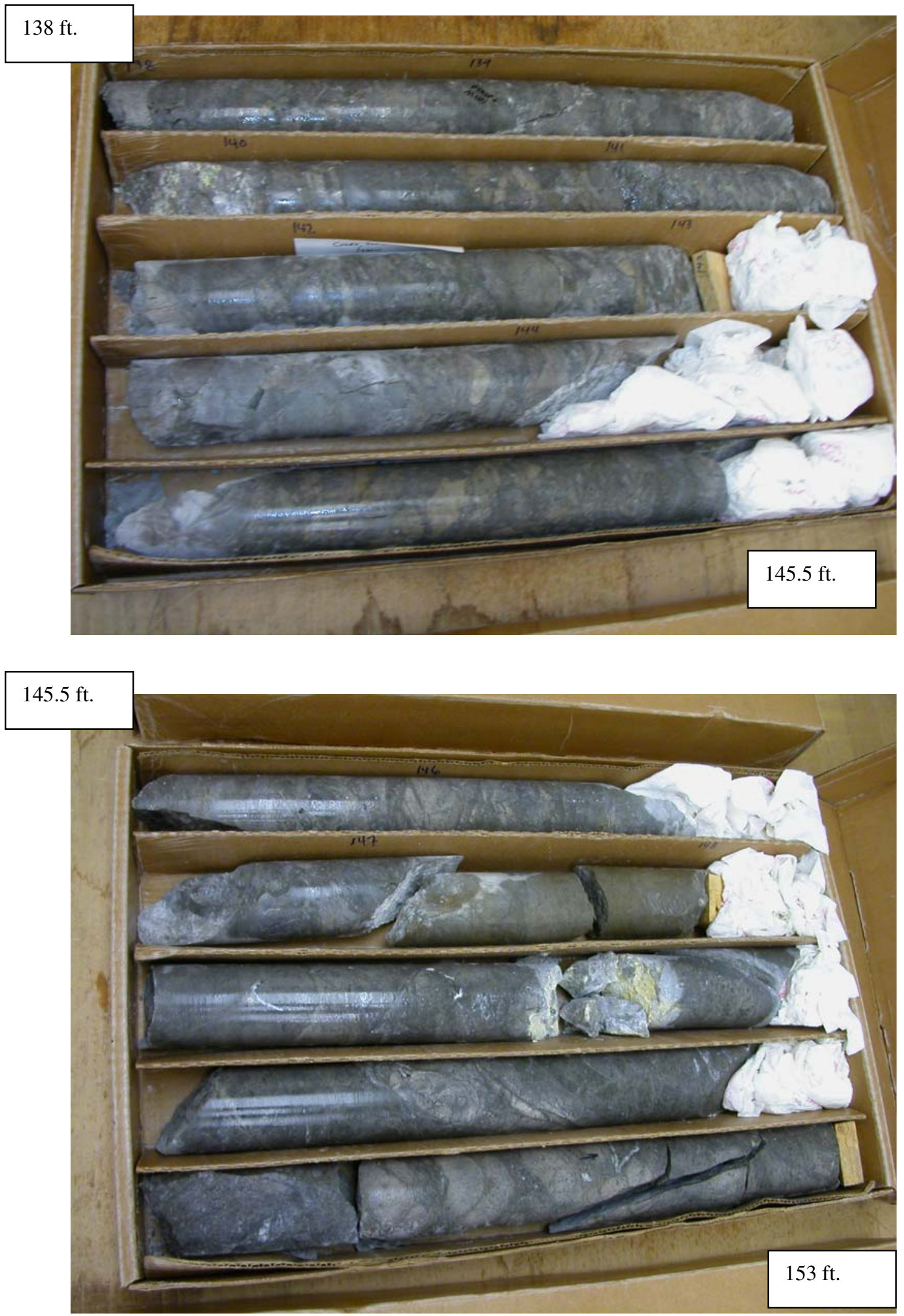


\section{Interval from 153 to $176.3 \mathrm{ft}$.}

Coarse-grained, clast-supported breccias are dominant from 153-157 ft. These coarse grained breccias have late, finegrained and prismatic quartz and some drusy quartz crystals lining vugs. The vugs are up $4-5 \mathrm{~cm}$ across; minor finegrained pyrite post-dates the quartz in these vugs. Interval from $157-176.3 \mathrm{ft}$. is characterized by fine-grained breccias and some brecciated lavas; the lavas are cut by abundant 1-3 mm pyrite veinlets. Rocks in this entire interval are highly silicified, strongly sericitized, and contain minor pyrophyllite. Late fracture-filling pyrophyllite becomes more abundant in the lower parts of this core interval. Fine-grained pyrite typically exceeds $8-10 \%$.

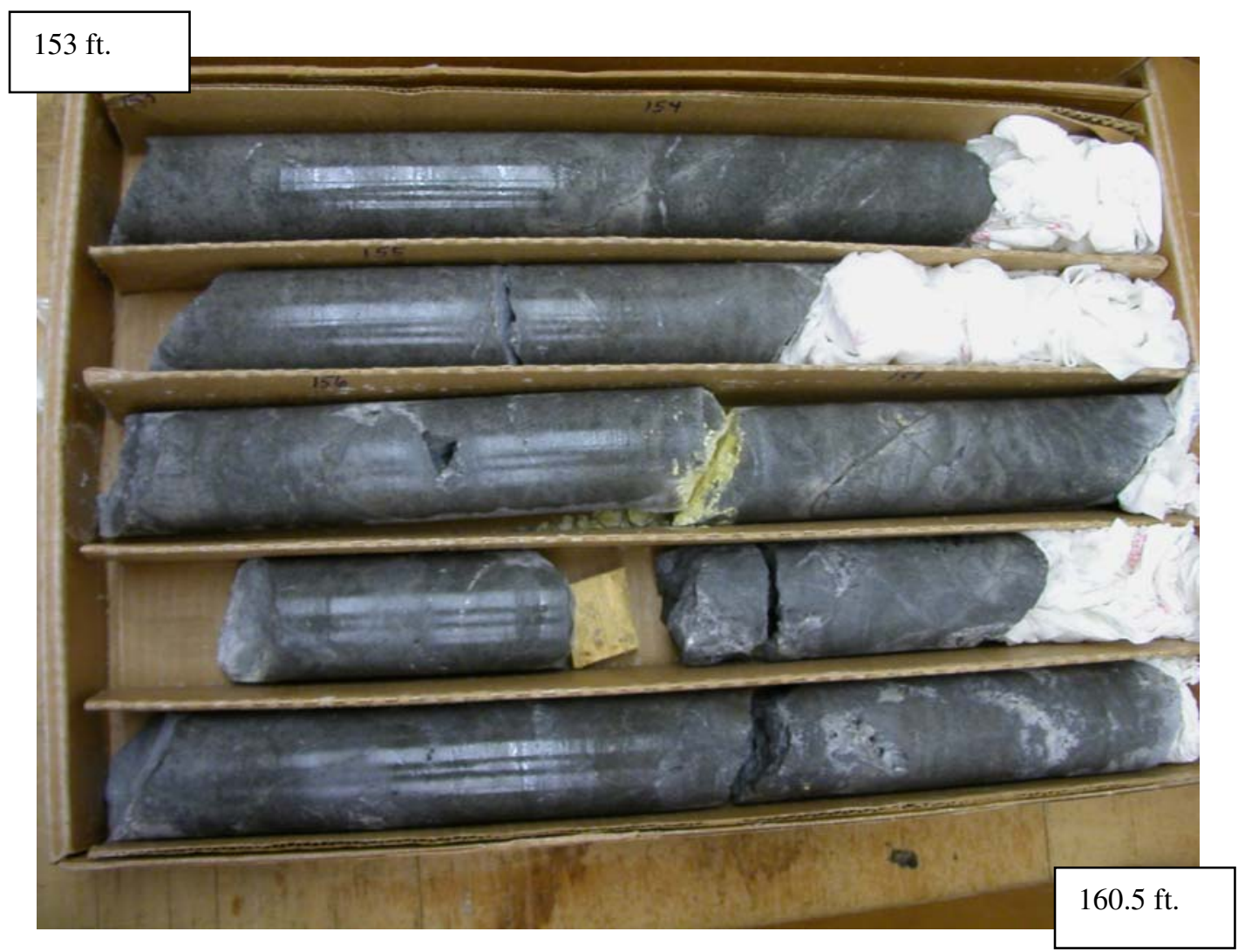



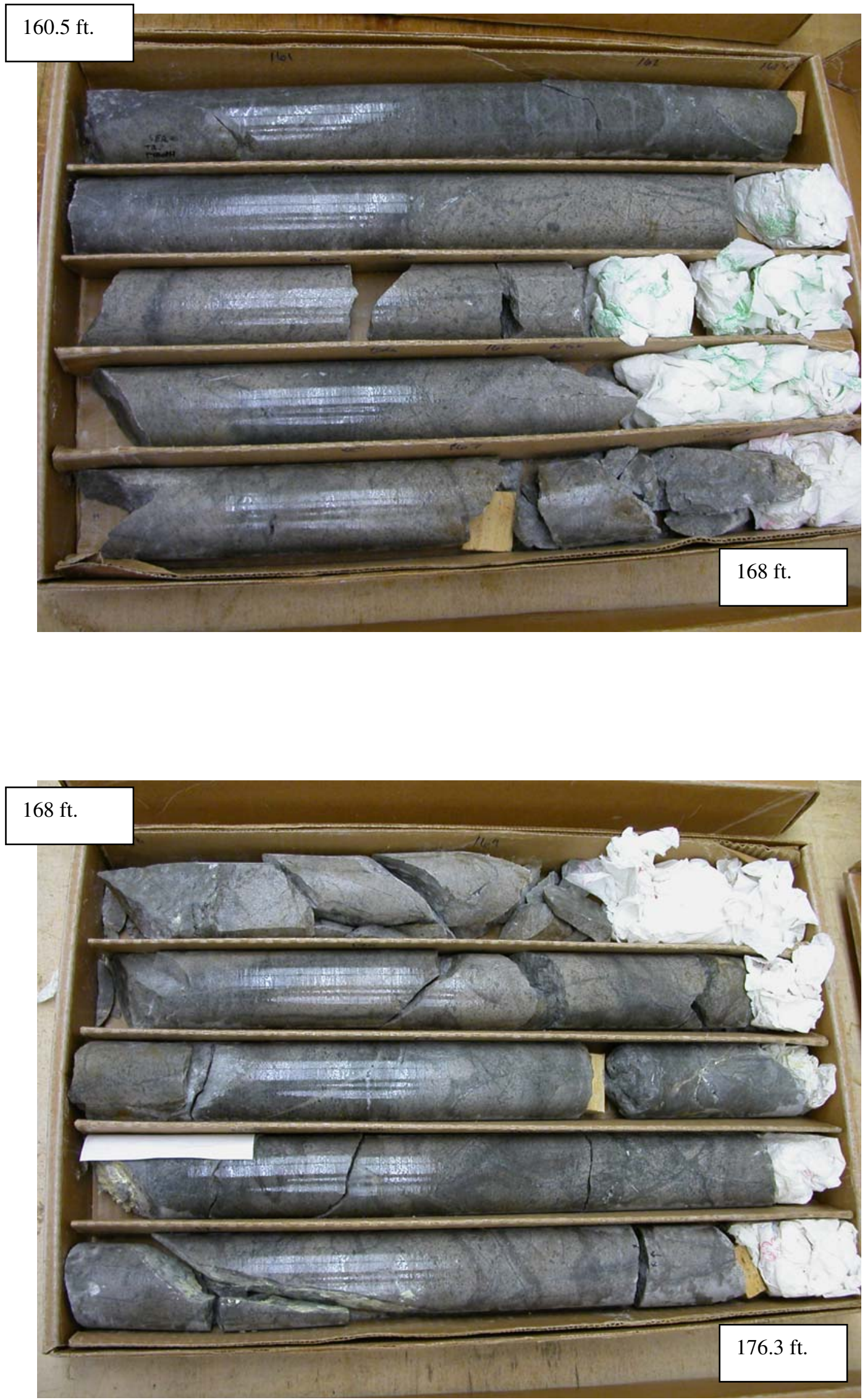


\section{Interval from 176.3 to $190.7 \mathrm{ft}$.}

This interval is mostly fine-grained lavas and brecciated lavas as above; however, breccias become slightly more prevalent. Rocks are highly silicified and sericitized, and contain minor pyrophyllite. Pyrite is abundant and occurs in clots after mafics and along tiny fractures and veinlets. The lavas textures are still easily discerned due to pyrite pseudomorphs after mafic phenocrysts. Pyrite content 10-12\%.
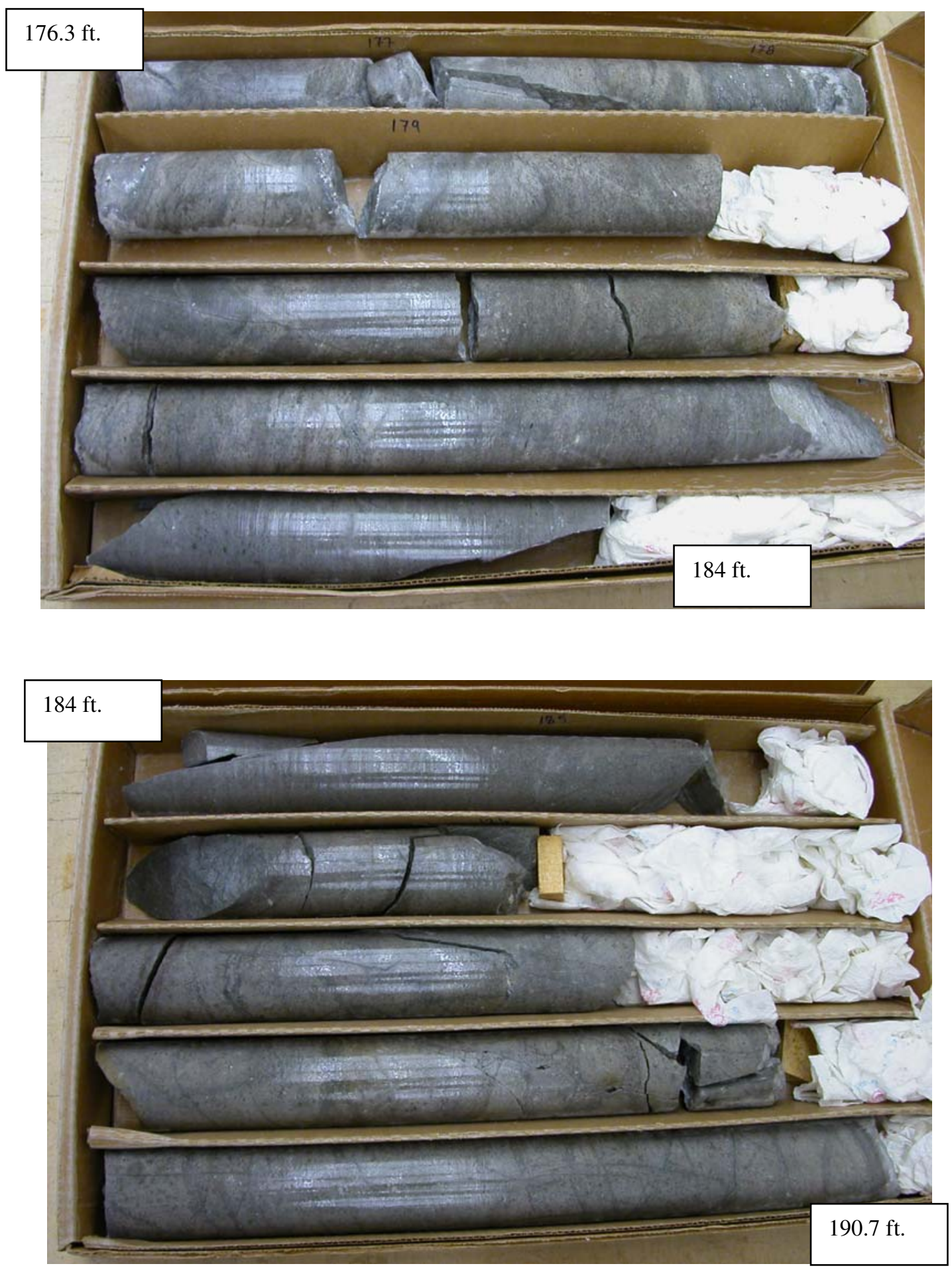


\section{Interval from 190.7 to $223.8 \mathrm{ft}$.}

Rocks comprised mostly of moderately to highly silicified, Qsp-altered lavas and brecciated lavas. The breccias are less abundant in this interval. Fine-grained, porphyritic textures are readily discerned in this interval. There are several intervals with stockwork pyrite veinlets averaging $1-3 \mathrm{~mm}$. There are a few zones that are more brecciated; these intervals are highly silicified and are commonly vuggy. The vuggy areas are similar to that described in the previous core intervals. There are several fracture zones filled with very fine-grained, white kaolinite/dickite. Several fractures also contain the late jarositic clay as described above. Fractures filled or lined by these late clays are mostly high angle.

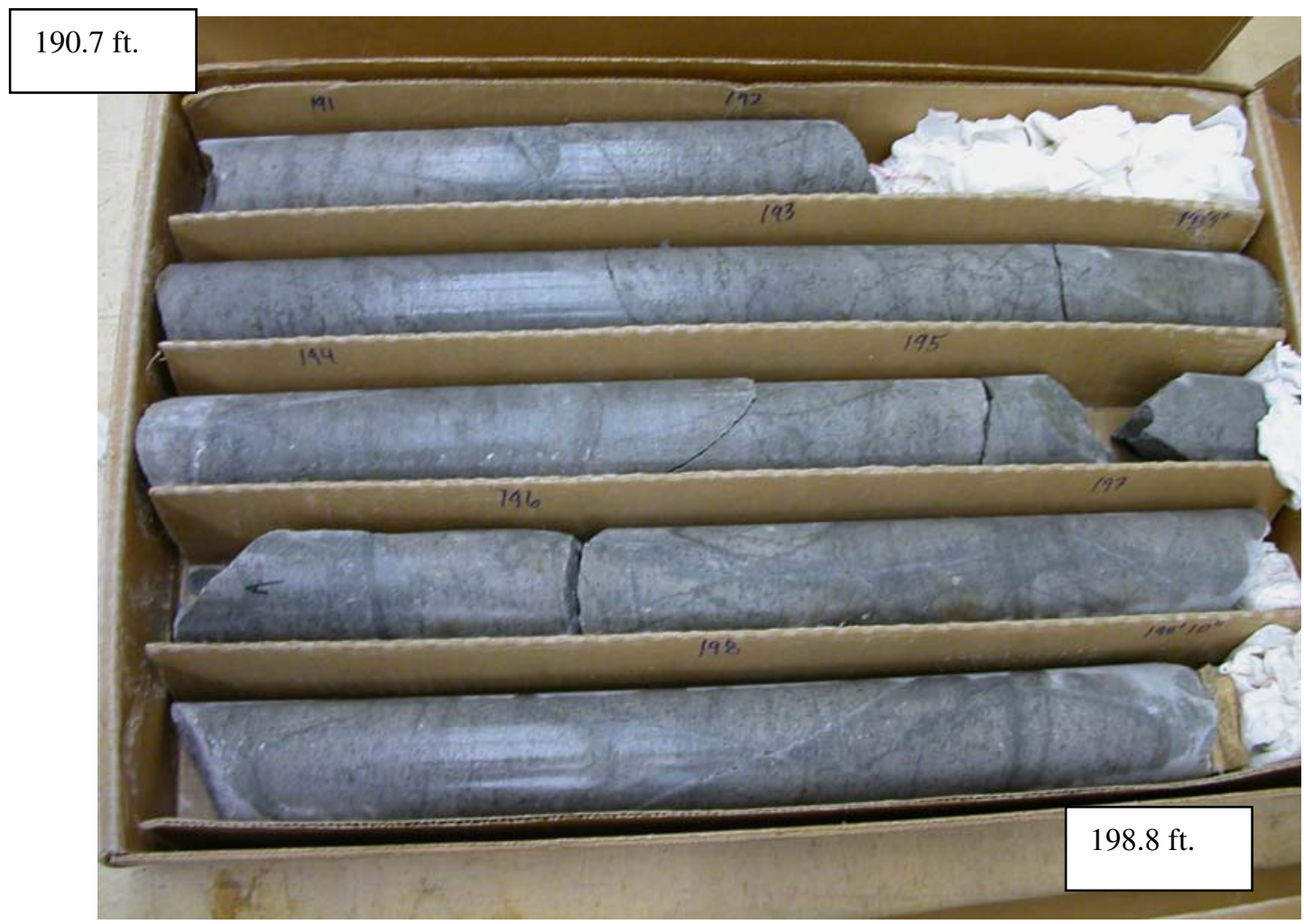



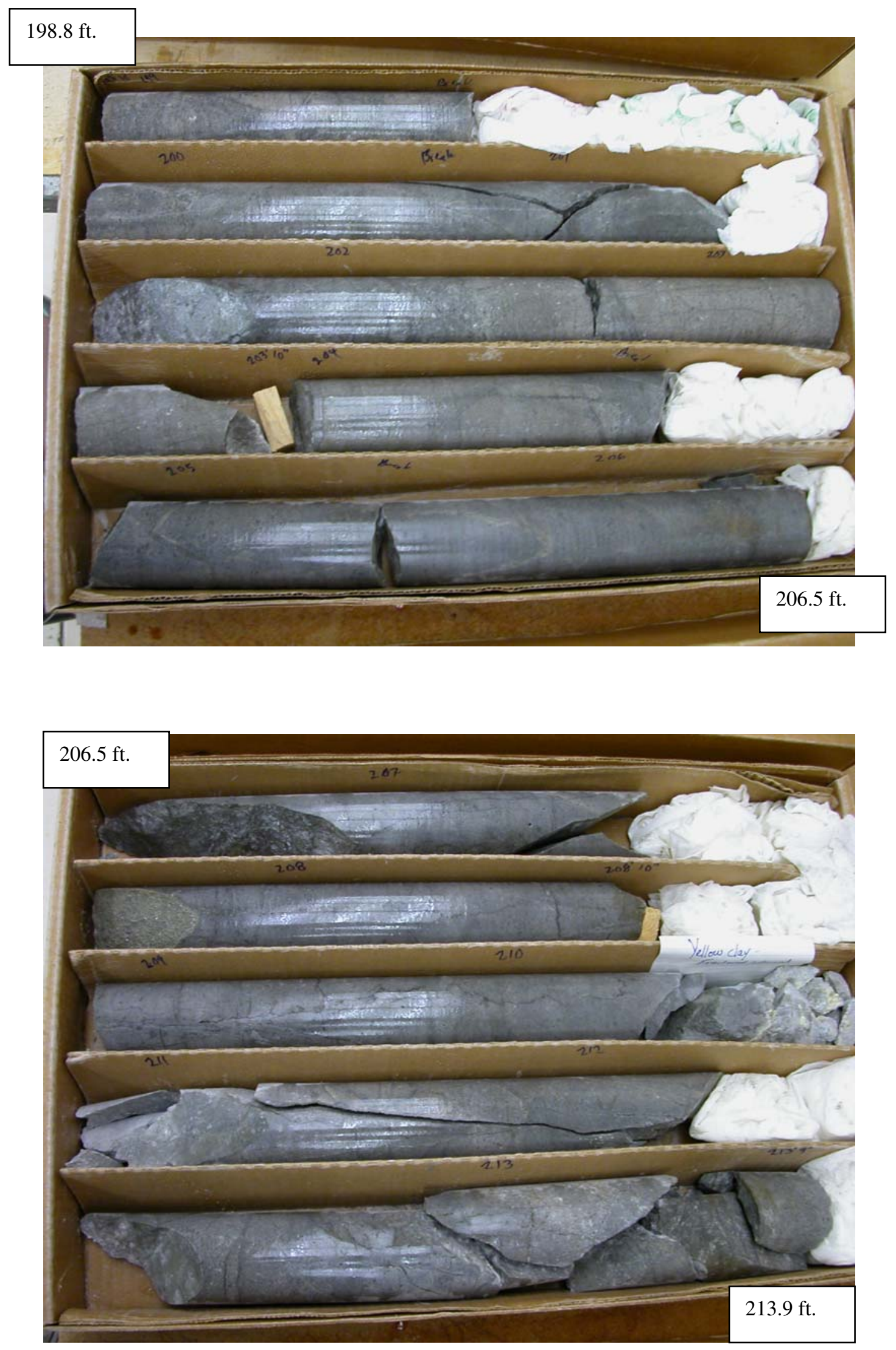

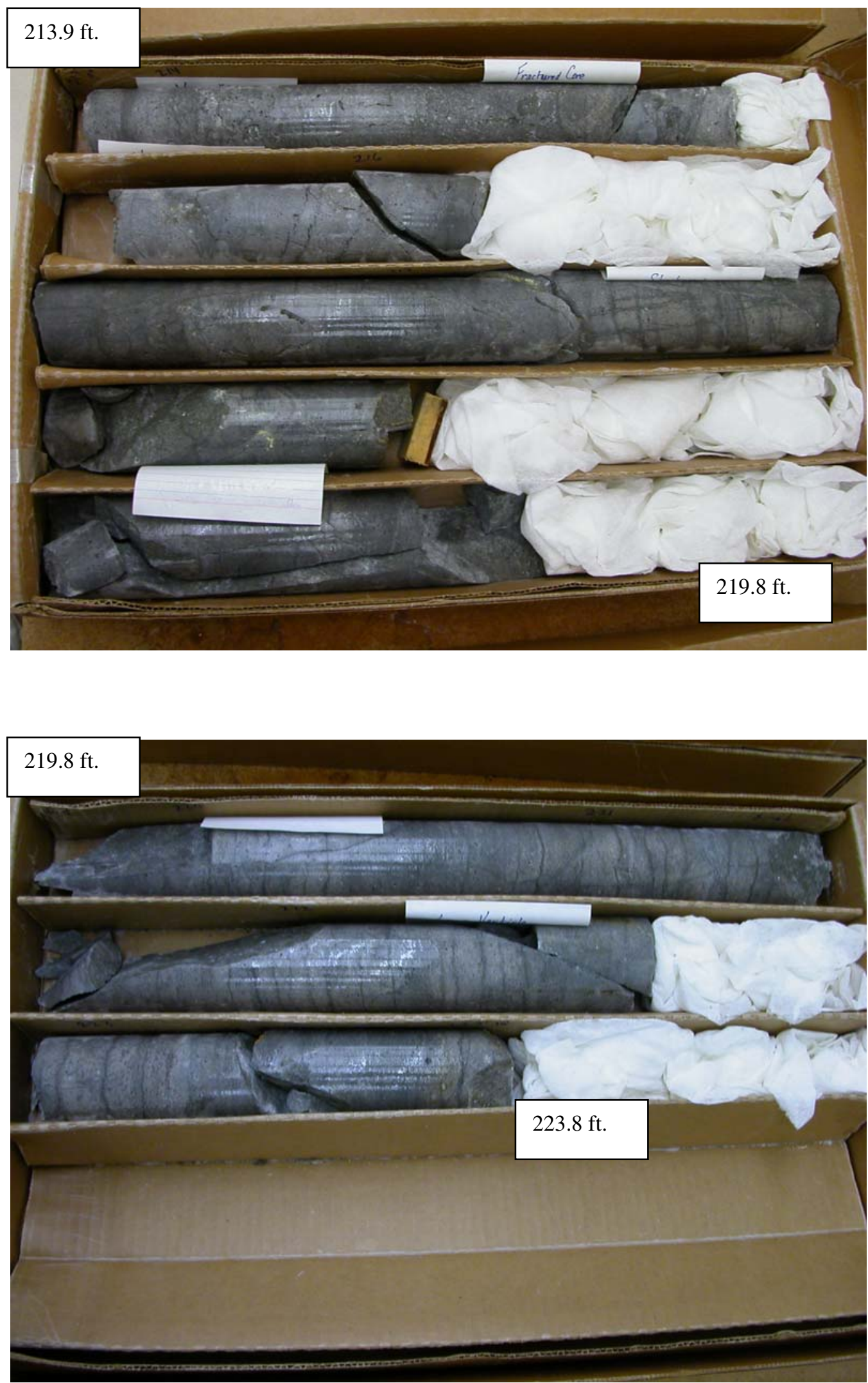


\section{Appendix B. Core log for MPG-D}

\section{Interval from 67 to $75.3 \mathrm{ft}$. (material above $67 \mathrm{ft}$. was unconsolidated and not cored)}

Entire interval is moderately silicified (50-55\% quartz), Qspaltered breccias. These rocks are overprinted by late-stage clay alteration comprised of pyrophyllite with minor dickite. Pyrite content averages about $10 \%$ in the unoxidized rock; sphalerite is locally abundant (4-5\%). Interval from 67-67.5 $\mathrm{ft}$. is jigsaw breccia with non-rotated and corroded clasts (Tsv lavas); open/vuggy areas in breccia matrix (2-5mm) filled by white pyrophyllite rich clay and pyrite. These open areas/vugs are filled with two generations of white clay. The earliest is snow white and somewhat waxy; this clay is comprised of an intimate mixture of dickite and kaolinite. A layer of paragenetically younger white clay (fine sugary appearance), containing only pyrophyllite, is the latest clay in this vug-filling sequence. The latest pyrophyllitic clay alternates paragenetically with an abundance of fine but euhedral pyrite. A few crystals of dark-green, fine-grained $(<2 \mathrm{~mm})$, botryoidal sphalerite, are latest in this vug-filling sequence. This breccia transitions into (or perhaps is cut by) matrix-supported breccia to $70 \mathrm{ft}$.; these breccias contain a few larger, subrounded, and more sporadic clasts $(1-2 \mathrm{~cm})$. These more coherent breccias lack open space or vugs and are cut by a few discontinuous white veinlets (1-3 mm) filled with white pyrophyllite \pm dickite. These breccias contain blebs of fine-grained pyrite and are cut by a relatively low density of pyrite veinlets as well. Interval from $70-72 \mathrm{ft}$. is comprised of coarser breccias as described above with late white clays in open matrix and a sphalerite-rich zone from 70 to $71 \mathrm{ft}$. Paragenesis is same as above, however the dark green sphalerite is much more abundant and is up to $0.75 \mathrm{~mm}$ in width. Rather than veins, theses sphalerite grains fill open space in the breccia matrix. The sequence here establishes that base-metal sulfide deposition followed brecciation and very late stages of acid-sulfate alteration. Rock from 71 to 75 $\mathrm{ft}$. is finer matrix-supported breccia and brecciated lavas. An uneven fracture $\left(\sim 25^{\circ} \mathrm{dip}\right)$ cuts the coarser breccias at $\sim 70 \mathrm{ft}$. and has a $2 \mathrm{~cm}$ iron-stained zone surrounding it. The fracture surface also localized a relatively thin clay-rich zone (1-3 $\mathrm{mm})$. Two small fractures with similar 20-25 degree dips localized oxidation at $71 \mathrm{ft}$. (very thin oxidation) and at 72 ft. (banded oxidation about $3 \mathrm{~cm}$ ). A very steep, clay-lined fracture cuts fine breccias/lavas at $73 \mathrm{ft}$. The fracture surface is clay-lined and strongly coated with goethite, jarosite, and hematite. The footwall of the fracture/fault localized highly clay altered rubble to $75 \mathrm{ft}$. Banded oxidation is present on both margins of this fractured zone.

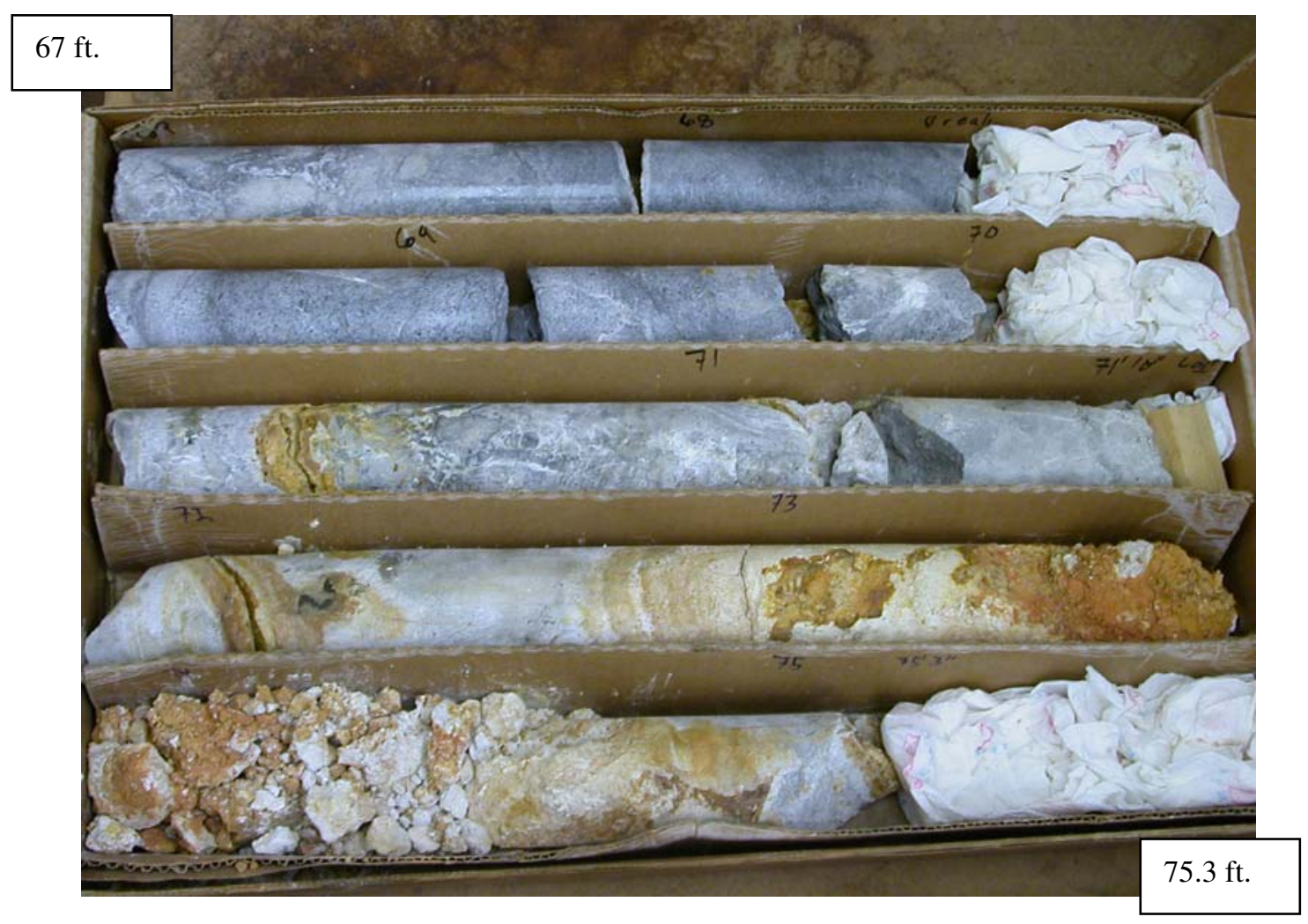




\section{Interval from 75.3 to $83.5 \mathrm{ft}$.}

Mix of alternating coarse and fine-grained breccias/brecciated lavas as described above. The breccias are all compact and tight without open space in matrix. This zone is slightly less silicified, and is not cut by late, white clay-filled veinlets. These Qsp-altered rocks are also overprinted by about $20 \%$ pyrophyllite and contain about $7-8 \%$ fine-grained pyrite. There are about five prominent fractures (30-50 dips, multiple directions) that localized jarositic and goethitic oxidation as described above $(75 ; 76.5 ; 77 ; 77.5 ; 78$ and $80 \mathrm{ft}$.). Fractures at 75 and $80 \mathrm{ft}$. localized clay and some breccia. Note that there is light gray oxidative bleaching $(1 \mathrm{~cm})$ around a couple of these fractures.

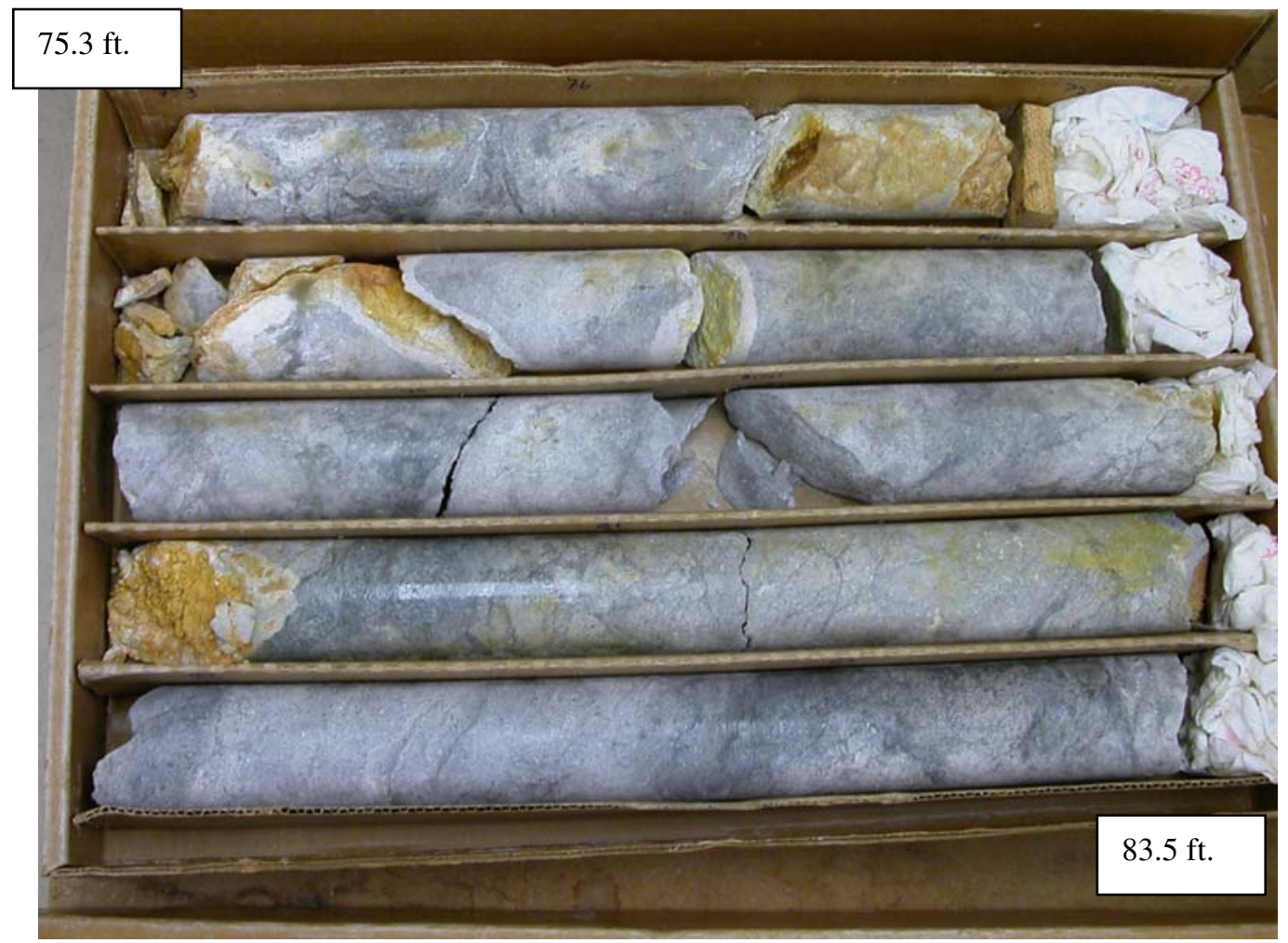




\section{Interval from 83.5 to $91.6 \mathrm{ft}$.}

Medium gray, moderately silicified, quartz-pyrophyllitealtered, coarse-grained and clast-supported breccias. There is very rare to minor amounts of sericite in this interval. Breccias are very complex, multigenerational, and clasts have a variety of shapes and are varied alteration types. Many of the clasts are subrounded to subangular; some have corroded margins. Pyrite concentration bands thinly surround many of the clasts. There is fine open space between some clasts, creating a slight vuggy appearance in places. White pyrophyllitic clay is prevalent as fillings in these spaces. White pyrophyllite and fine pyrite are abundant and form tight fillings throughout much of the breccia matrix. Moderately dipping fracture zones with iron staining and clay alteration are present at 85 and $86.8 \mathrm{ft}$. Two shallow dipping fractures localized gray clay alteration, but no oxidation occurs near $88 \mathrm{ft}$. Fine-grained pyrite in this interval averages about $5-6 \%$ and is present mostly in the breccia matrix but rarely in veinlets.

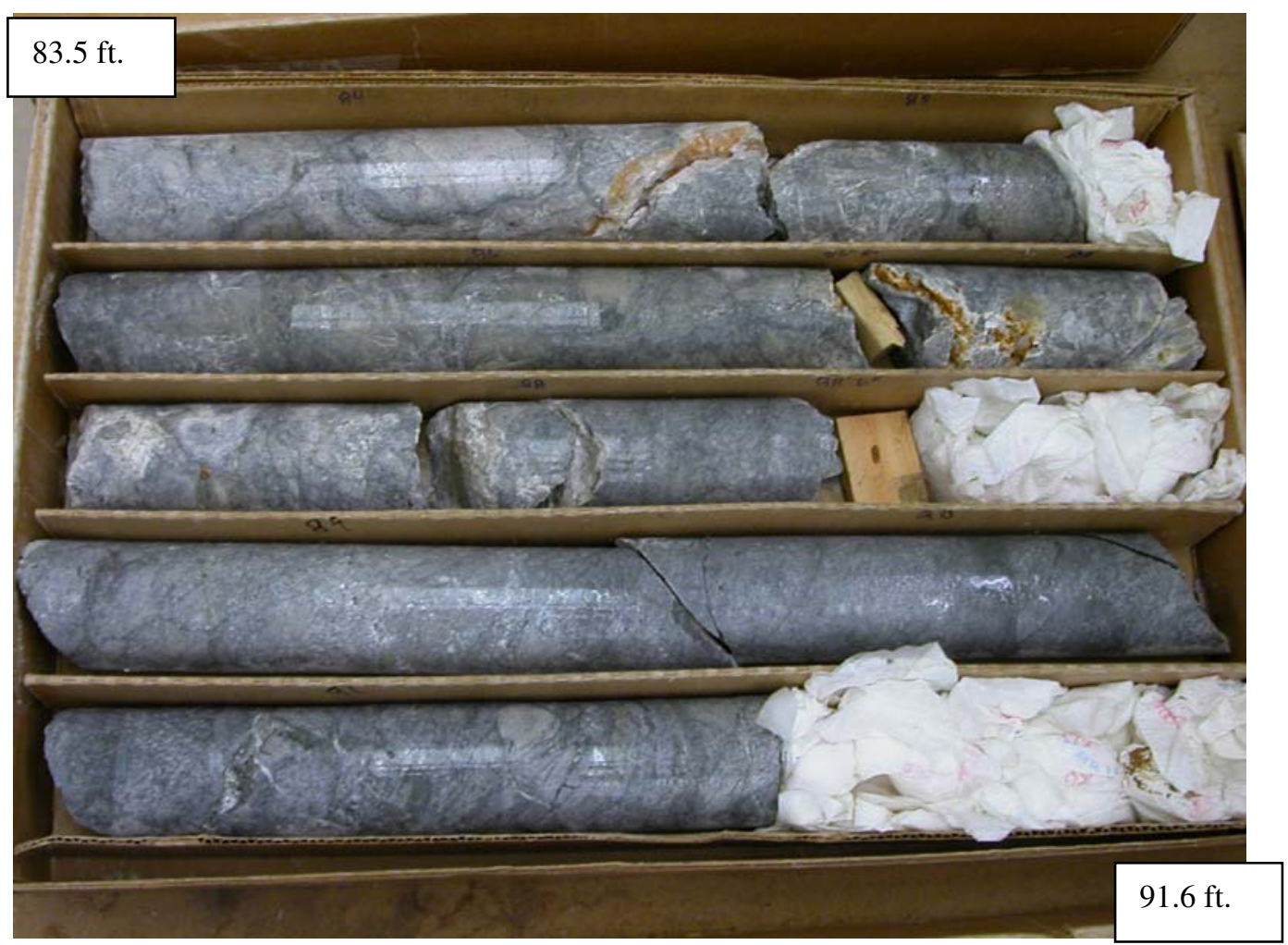




\section{Interval from 91.6 to $99.3 \mathrm{ft}$.}

Breccia from above interval almost immediately transitions into light to medium gray, Qsp-altered and mildly silicified lavas (Tsv). This core interval has some very interesting textures created by diffuse pyrite flooding/alteration that localized along near vertical fractures; these diffuse zones are as much as $1 \mathrm{~cm}$ wide. The dark, near vertical pyritic zones branch into similarly wide and diffuse zones; these zones are at low angles to the core. These diffuse zones are subparallel to a low to moderate density of thin pyritic veinlets (1-3mm) that have more defined margins. The orientation of these veinlets/pyrite zones are likely subparallel to foliation. The fine porphyritic texture of the lavas is moderately obscured and matrix pyrite is in small blebs (1-2 mm). Average pyrite content in this zone is $5-8 \%$.

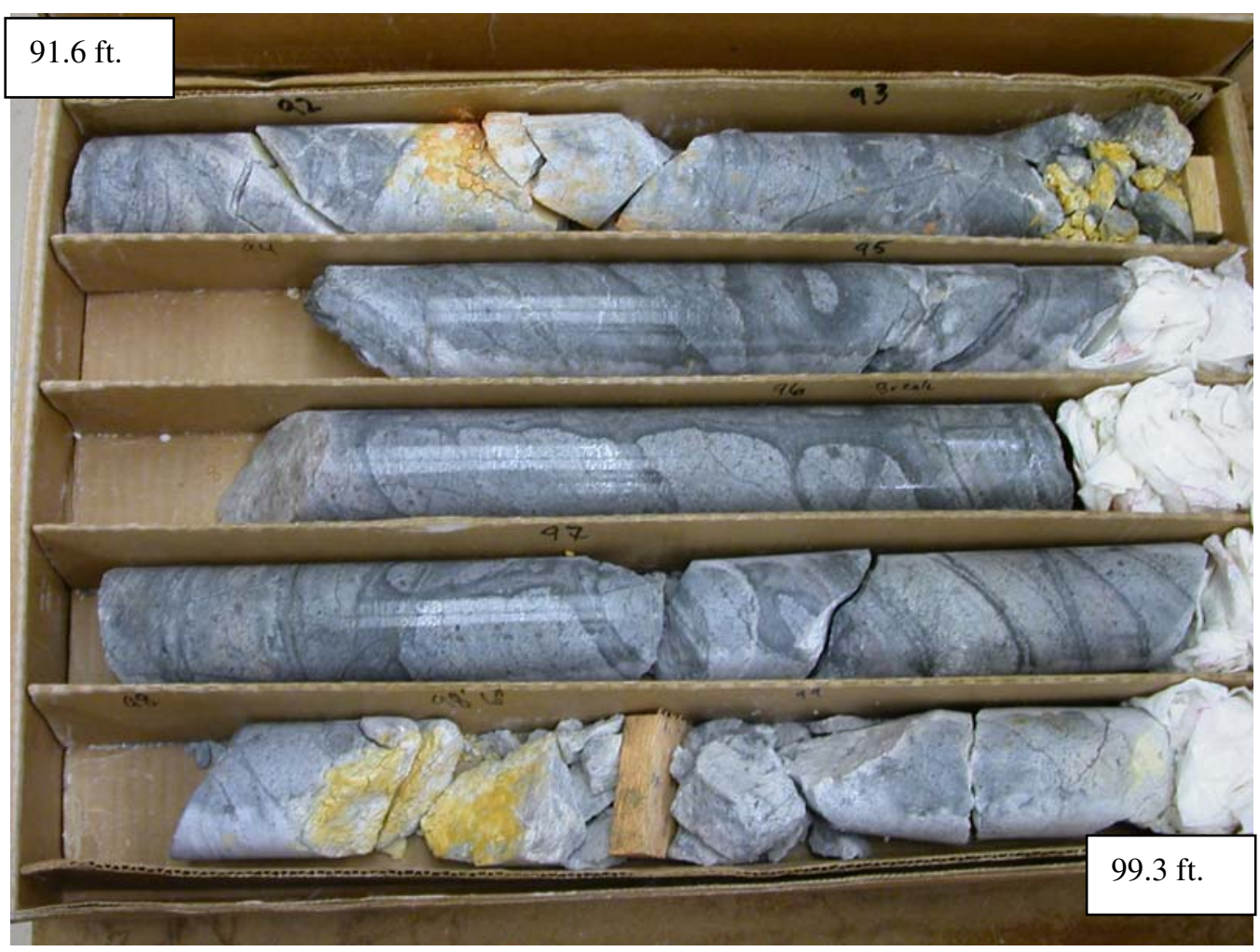




\section{Interval from 99.3 to $106.8 \mathrm{ft}$.}

Interval is fairly similar to previous, however density of pyritic veinlets is increased and orientations may be in more conjugate sets. A very steep fracture zone with some clay and breccia cuts the core between 101 and $102 \mathrm{ft}$. The fracture is lined with medium gray clay. Another more moderately dipping fracture at $105 \mathrm{ft}$. separates altered lavas in the hanging wall from clast-supported breccia in the footwall to 106.8 $\mathrm{ft}$. The fracture is very sharp and has localized whitish-gray clay (dickite with pyrophyllite) and some breccia in this $1-2 \mathrm{~cm}$ zone. Several other fractures faces in this interval have localized thin white clay comprised of dickite, pyrophyllite, and minor sericite. These fracture faces have also localized abundant fine-grained pyrite and very fine-grained sphalerite. Pyrite content in this interval averages $8-10 \%$.

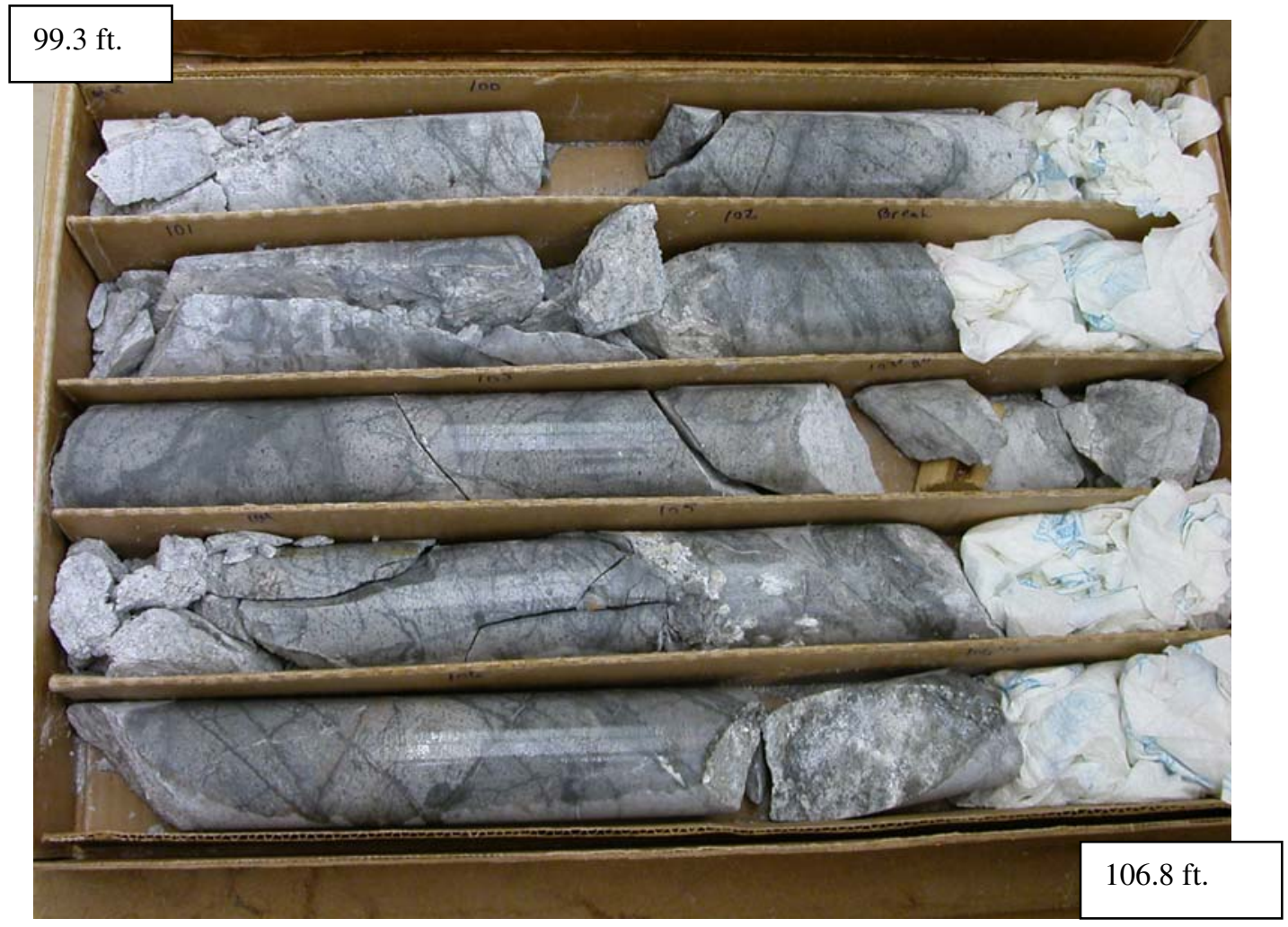




\section{Interval from 106.8 to $115 \mathrm{ft}$.}

Medium gray, Qsp-altered lavas (Tsv) are dominant to 111 ft. Textures are somewhat similar to that in interval from 93.7 to $99.4 \mathrm{ft}$. Qsp-altered breccias ranging from matrix to clast-supported are present in interval from 111 to 115 ft. Pyrite flooding is more abundant throughout this breccia interval. White veinlets of pyrophyllite/dickite sporadically cut the entire core interval. Pyrophyllite alteration generally becomes more abundant from top to bottom of entire core interval. Pyrite content increases from about $5 \%$ in altered lavas to about $10-12 \%$ in the breccias.

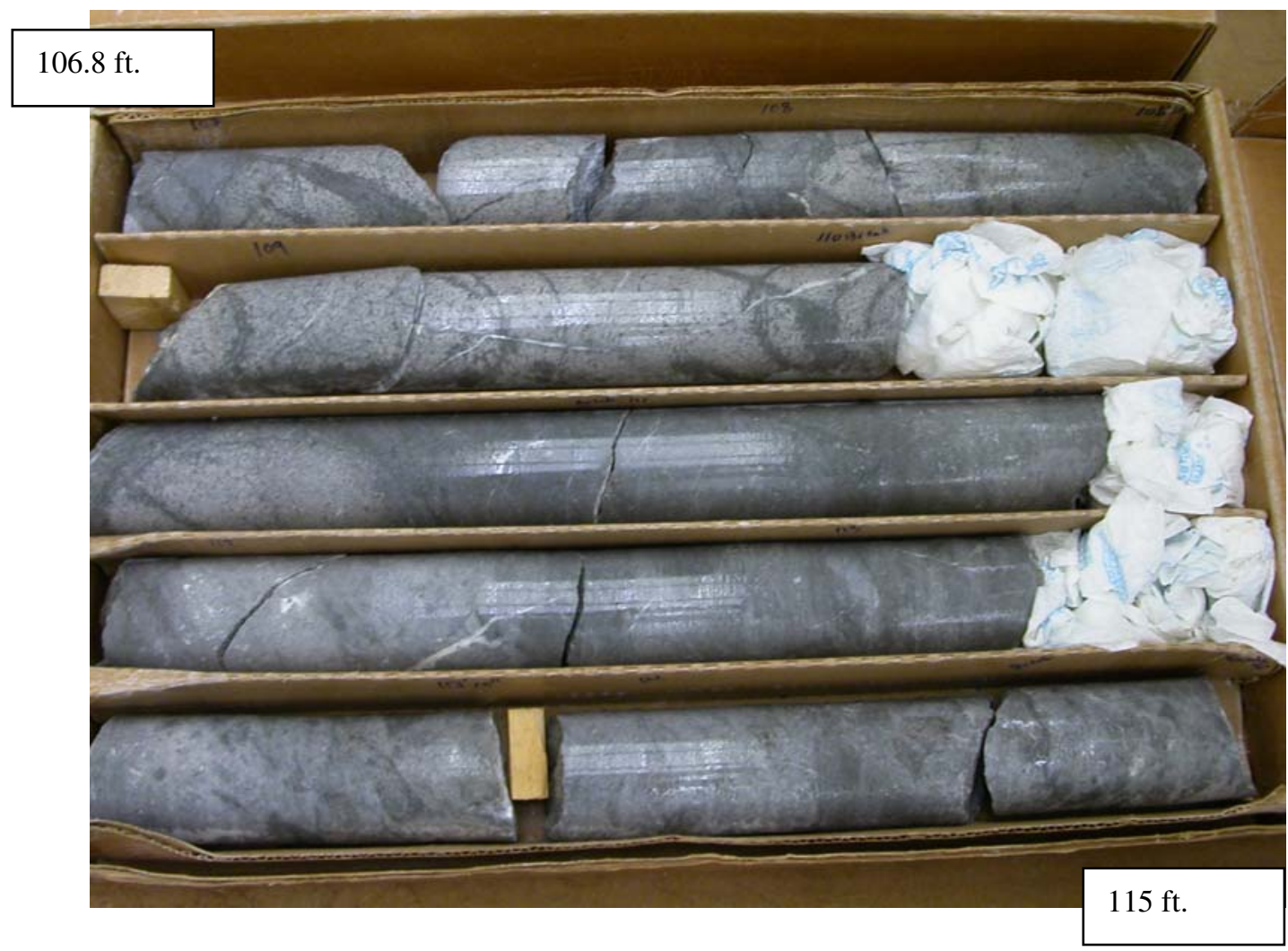




\section{Interval from 115 to $122 \mathrm{ft}$.}

Very dark gray-blue, strongly pyrophyllite-altered assortment of hydrothermal breccias. Breccia textures and sizes are typical of above zones, tending toward more coarse-grained and clast-supported. Rocks are very soft and easily scratched owing to pervasive replacement by pyrophyllitic clay. A few discontinuous white pyrophyllite/dickite veinlets cut the breccia Fine-grained pyrite is abundant in this interval and ranges from $10-15 \%$.

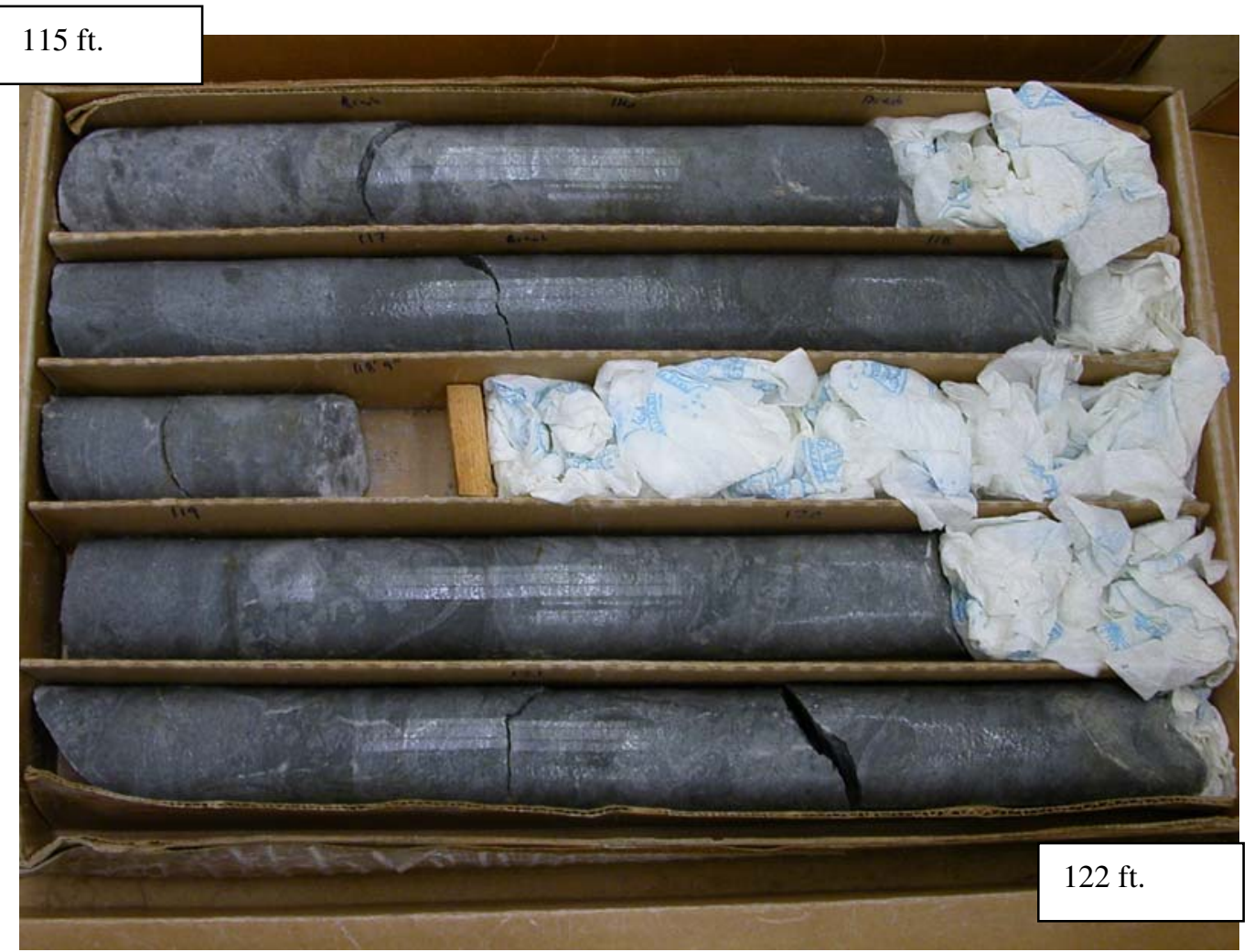




\section{Interval from 122 to $130 \mathrm{ft}$.}

Medium gray to gray-blue, Qsp and pyrophyllite-altered breccias; low to moderate silicification. Clast-supported breccia is darker gray (due to higher pyrite concentrations) and has entrained larger clasts of altered Tsv lavas $(0.2$ to $0.3 \mathrm{~m})$. The clast-supported "matrix" that supports the larger clasts contains variously altered, subrounded to subangular clasts $(4-8 \mathrm{~mm})$. Fine-gained pyrite is localized in the matrix of these breccias and in places outlines the larger clast margins. The Tsv clasts are characterized by evenly distributed pyrite blebs $(<1 \mathrm{~mm})$. White pyrophyllite/dickite and associated fine pyrite in-filled breccia void space in the interval around $126 \mathrm{ft}$. Wispy and discontinuous veinlets of the same white clay are also common in the entire core interval. Pyrite content is roughly $8-10 \%$.

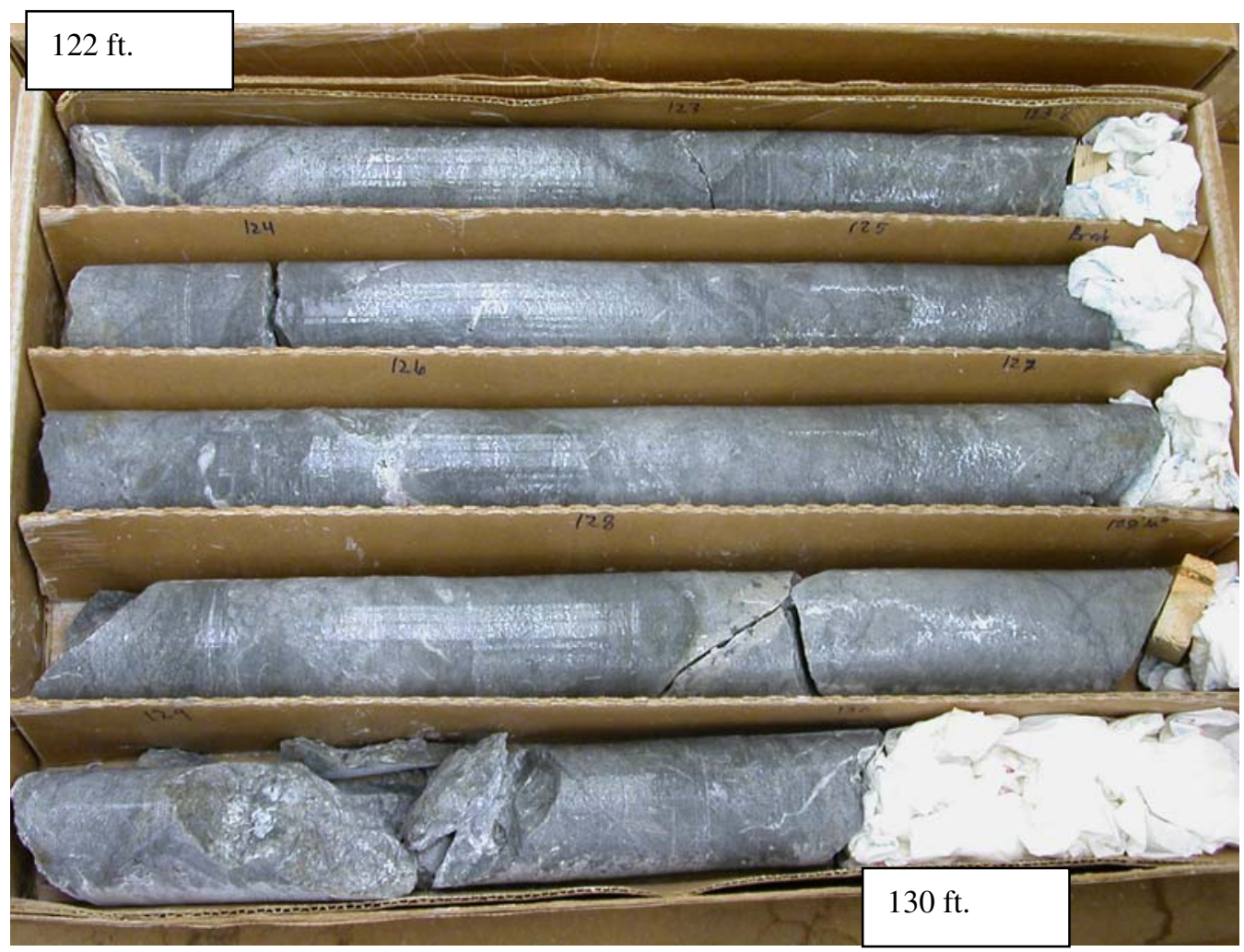




\section{Interval from 130 to $137.3 \mathrm{ft}$.}

Breccias continue as above but are very dark gray due to increase in silicification (50-55\% quartz. Interval is dominantly Qsp-altered with less pyrophyllite (similar to that described above). Rock is locally vuggy and these open spaces are either empty or filled with white clay (pyrophyllite). Several high-angle fractures at present at 132; 134; 136 and $137 \mathrm{ft}$ with some slickensides and later filling by white clays. Pyrite content ranges from 7-10\%.

Sample at 136' has an important paragenesis: Dark Qspaltered and more silicified breccia containing very fine pyrite (or a very large clast of previously brecciated rock) is cut by or perhaps supported in another breccia (clay alteration is possibly sericite). The later breccia (or matrix to the coarse breccia clast) is healed and/or replaced by a flesh colored mixture of pyrophyllite and minor natroalunite (if pyrite is present, it is very fine grained, sparse, and intermingled in alunite). All are cut by a $1-2 \mathrm{~mm}$ fracture filled with white pyrophyllite. The white clay in this and similar late fractures alternate paragenetically with abundant fine-grained pyrite. This late generation of pyrite accounts for the majority of pyrite in this interval.

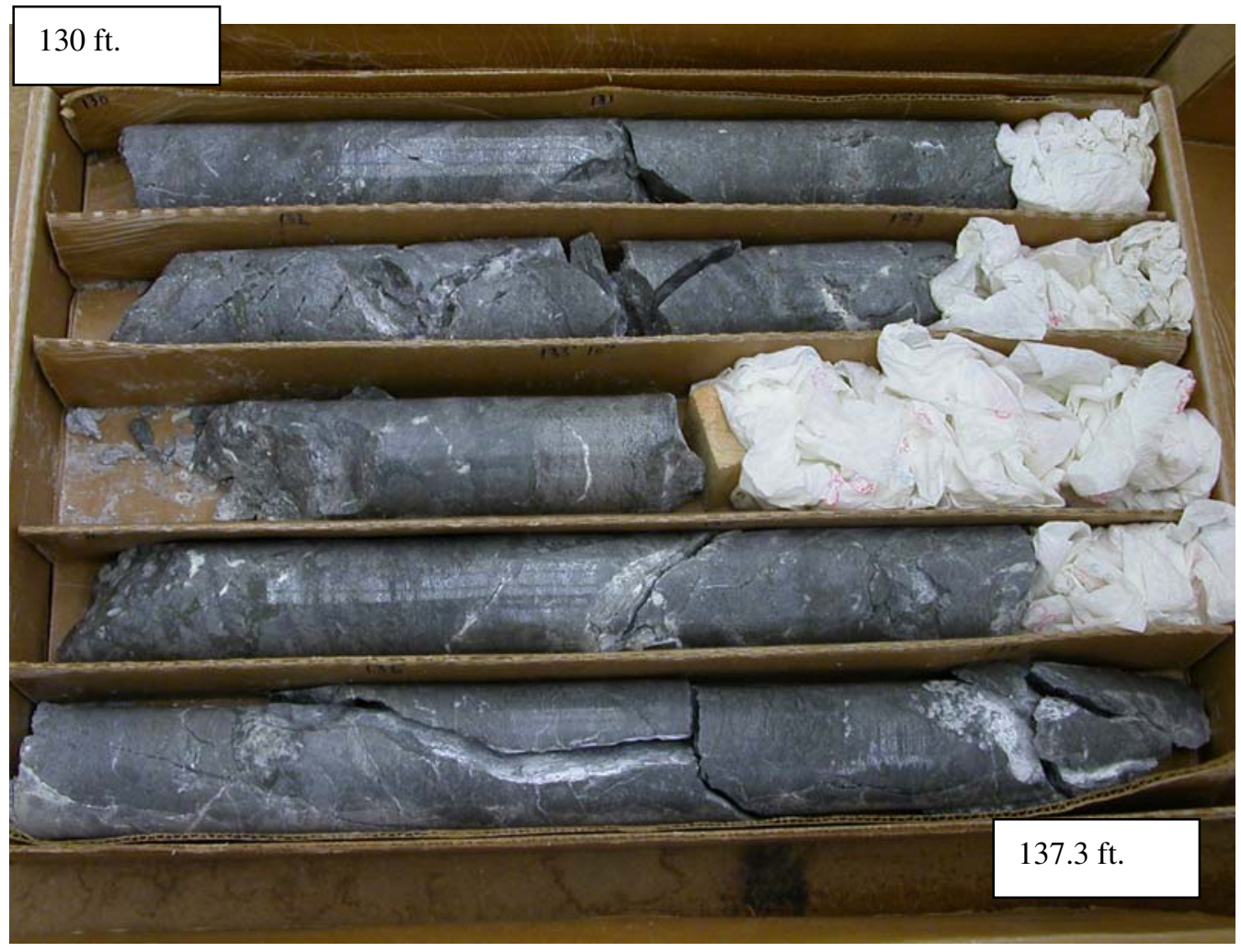




\section{Interval from 137.3 to $145.8 \mathrm{ft}$.}

Breccia similar to that described above to $138.5 \mathrm{ft}$. Interval from 138.5 to $145.8 \mathrm{ft}$. is cut by four prominent fractures. Three of these are associated with clay-altered breccias and broken zones. Clay-altered breccia zones localized late pyrophyllite alteration. A high angle fracture with a smooth clay face is present at $138.5 \mathrm{ft}$. There is a slightly broken and fractured zone filled with white pyrophyllite clay $(2-3 \mathrm{~mm})$ that is concentrated along the fracture surface. The high angle fracture intersects a near horizontal fracture at $138.3 \mathrm{ft}$; this fracture has a $2 \mathrm{~cm}$ brecciated and clay altered zone that localized later pyrophyllite. There is a relatively undisturbed wedge of silicified rock between the two fractures. A $45^{\circ}$ fracture at $143 \mathrm{ft}$. is lined by thin, white pyrophyllitic clay but no associated breccia. A highly broken, brecciated and fractured zone is present from 144 to 145 ft. This zone has fragments with intervening clay altered and finely brecciated matrix that is overprinted by late pyrophyllite. Pyrite from ranges from about $8-10 \%$ in entire interval; sericite predominates over pyrophyllite in the entire interval.

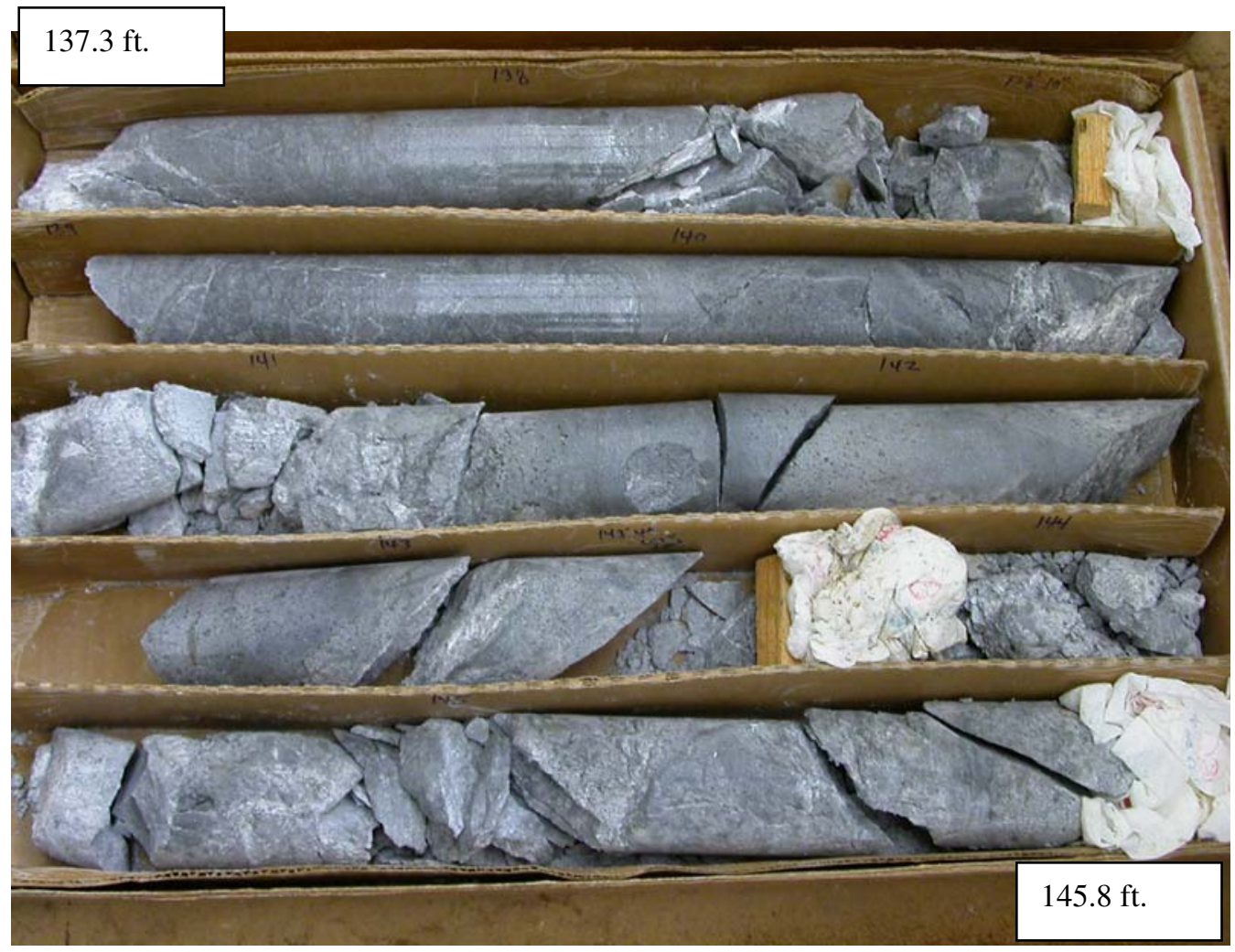




\section{Interval from 145.8 to $154.8 \mathrm{ft}$.}

Entire interval is highly broken and shattered; broken and clay-lined fracture surfaces noted throughout. See descriptions for broken zones in above interval (137.3 to $145.8 \mathrm{ft}$.).

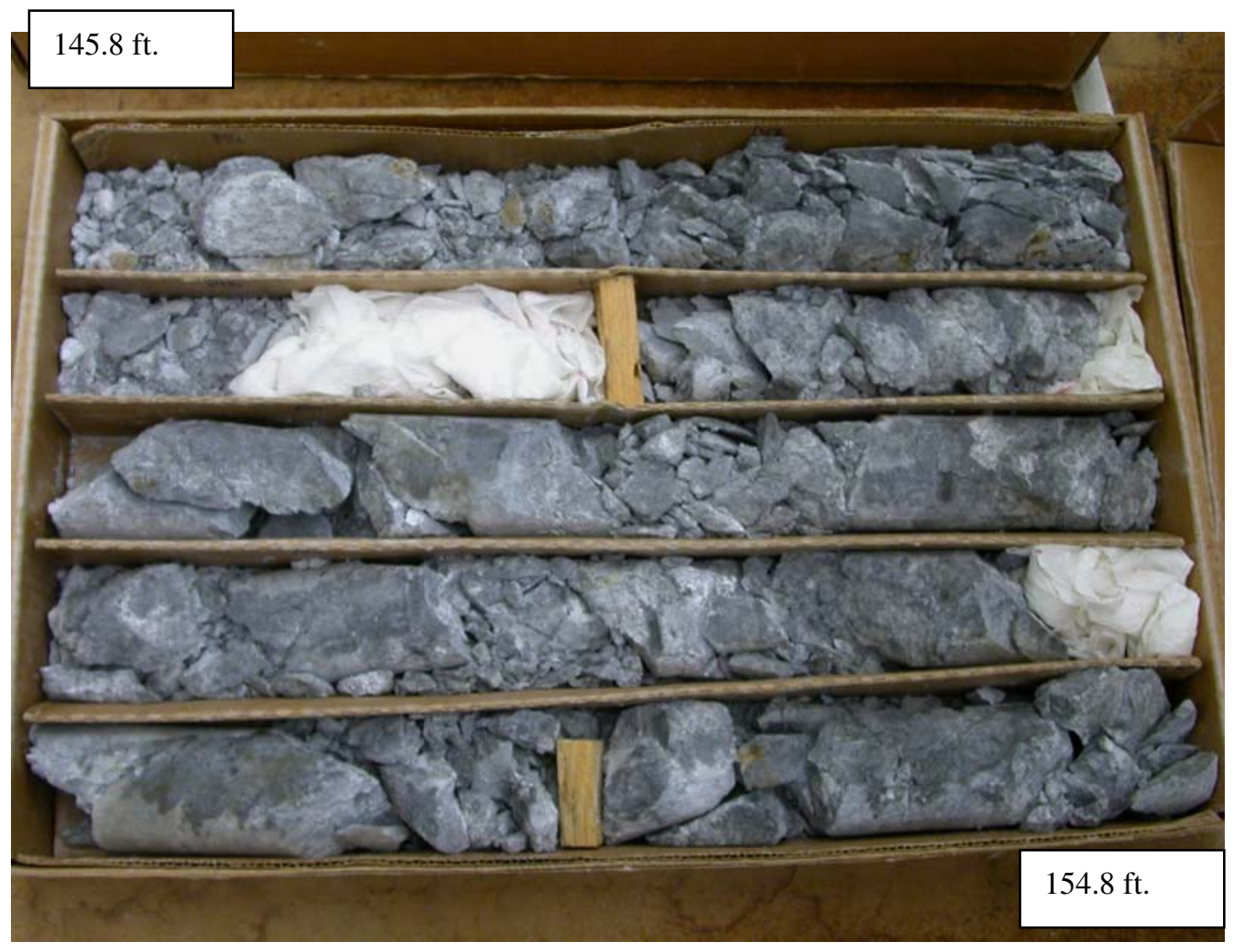




\section{Interval from 154.8 to $163 \mathrm{ft}$.}

Dark gray, clast-supported breccias with larger Tsv lava clasts (4-6 cm). Remnant texture of breccias and Tsv clasts is still discernible. Breccia that is present between larger Tsv clasts is darker due to a greater abundance of fine pyrite. Darker and more indurated breccias are moderately to highly silicified and Qsp-altered; lighter and more broken zones are Qsp-altered, minor to moderately silicified, and are overprinted by more pyrophyllite. A few broken zones are localized about fractures; minor to moderate density of discontinuous, white, pyrophyllite veinlets cut the breccias. Pyrite content varies from 12-14\%.

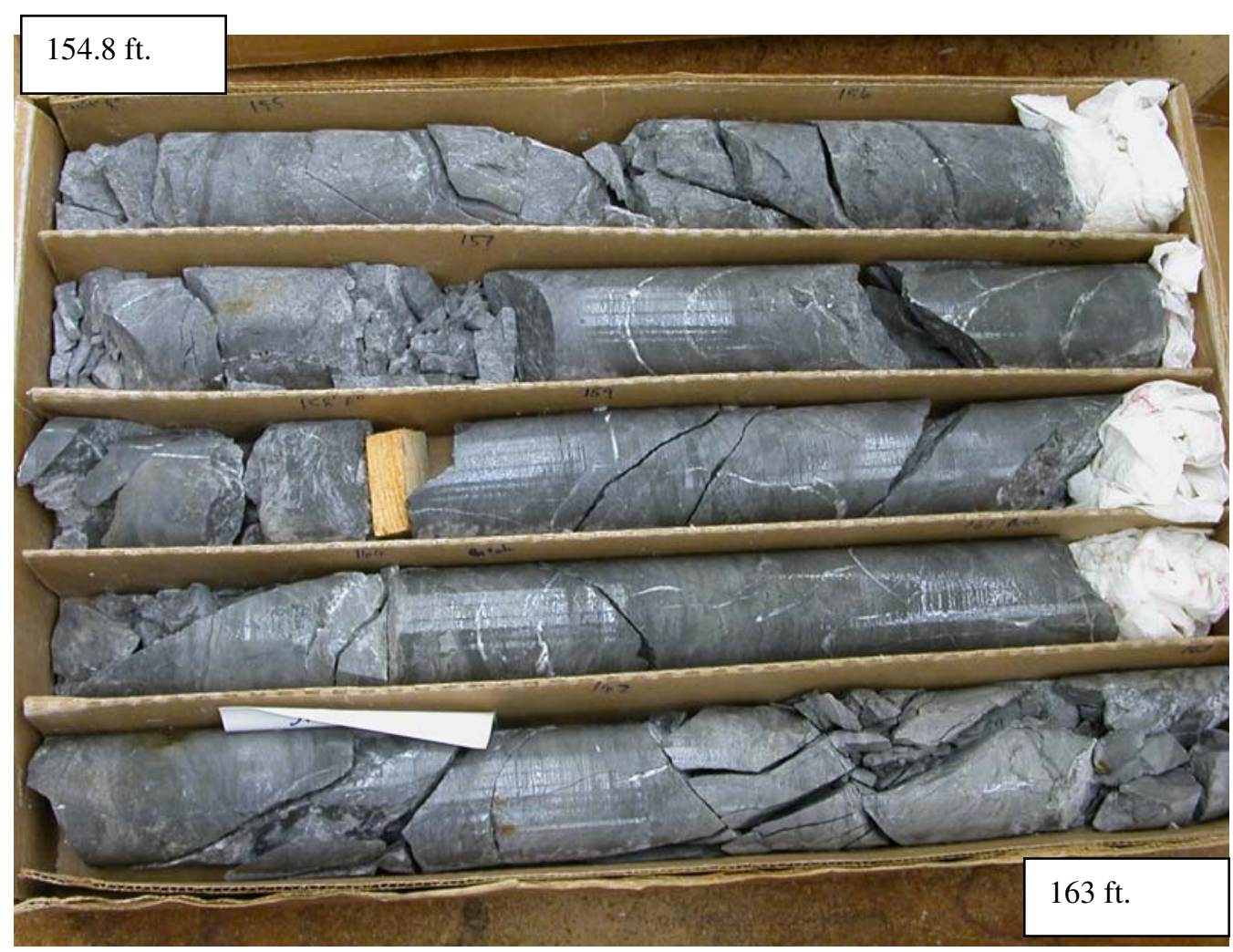




\section{Interval from163 to $173 \mathrm{ft}$.}

As above to $167 \mathrm{ft}$. Exceptions are that Tsv clasts are even larger-still suspended in breccia as described above. Rocks to $167 \mathrm{ft}$. are dark-gray, Qsp-altered, and contain 12-14\% finely disseminated pyrite. Breccias are mostly soft, broken rubble to $173 \mathrm{ft}$. A very high angle fracture spans interval from 172-173 ft.; another at $172 \mathrm{ft}$. is likely associated with rubble and clay altered brecciation (breccias and rubble may be fracture/fault related and alteration post-dates brecciation). Rubble zones have minor pyrophyllite overprint. Entire interval averages $10-12 \%$ fine-pyrite.

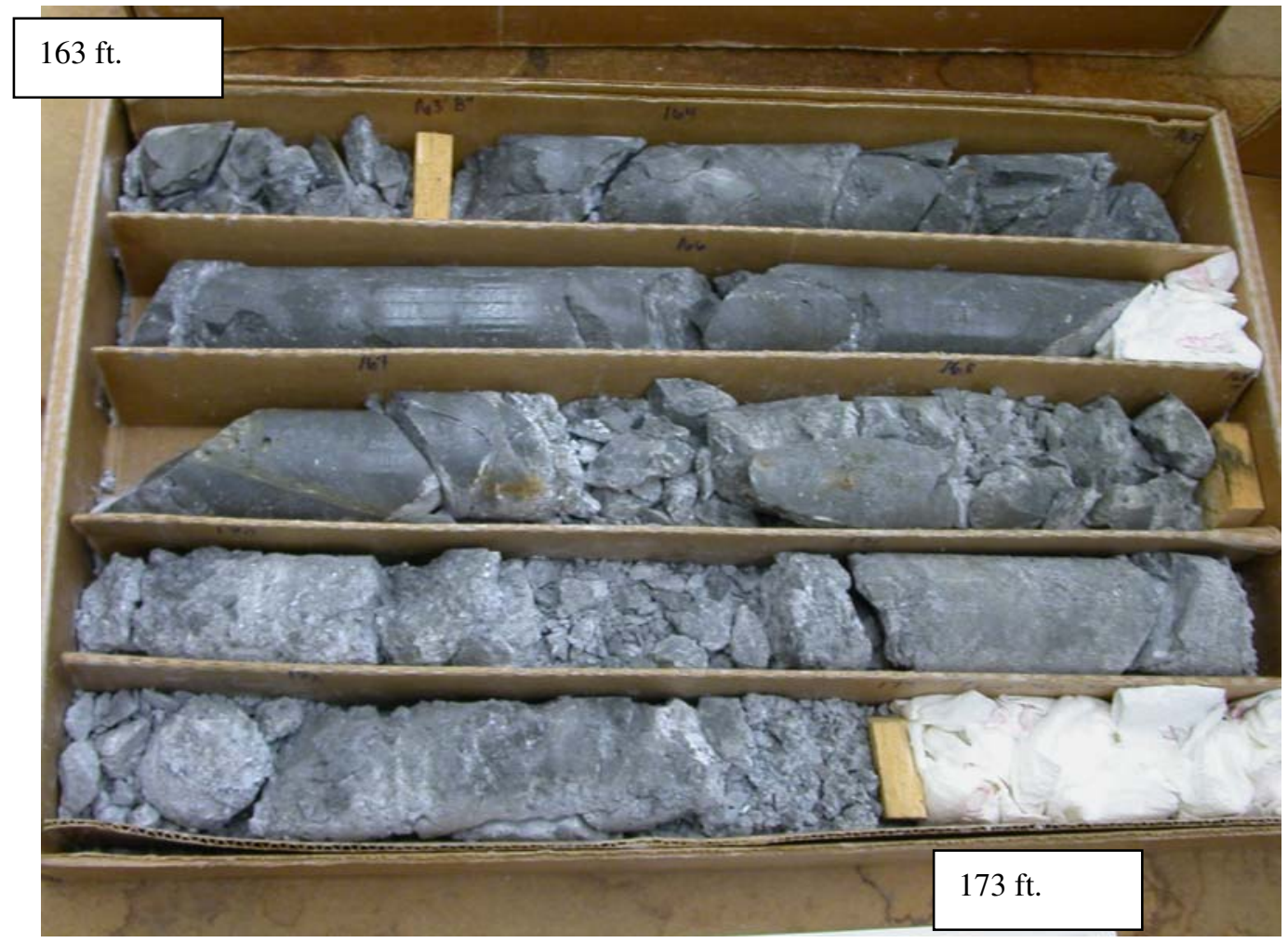




\section{Appendix C. Core log for LPG-D}

Interval from 8.3 to $13.6 \mathrm{ft}$. (material above $8.3 \mathrm{ft}$. was unconsolidated and not cored)

Zone with coarse alluvial ferricrete. Contains rounded to subrounded, cobble and pebble-sized clasts cemented by massive to laminated iron-oxide matrix. XRD confirms crystalline iron-oxide phases in matrix to be almost entirely goethite; schwertmannite is not present. Clasts are predominantly Tsv porphyritic lavas that range from propylitic to weak sericitic-altered. Mafics in weak sericitic-altered clasts have altered to epidote and chlorite; plagioclase is metastable with some sericitic alteration. There is some bleaching of fresher Tsv lava clasts marginal to more matrix supported ferricrete zones. This is related to acid weathering; there is generally no discernable change in clay alteration in these more bleached intervals.

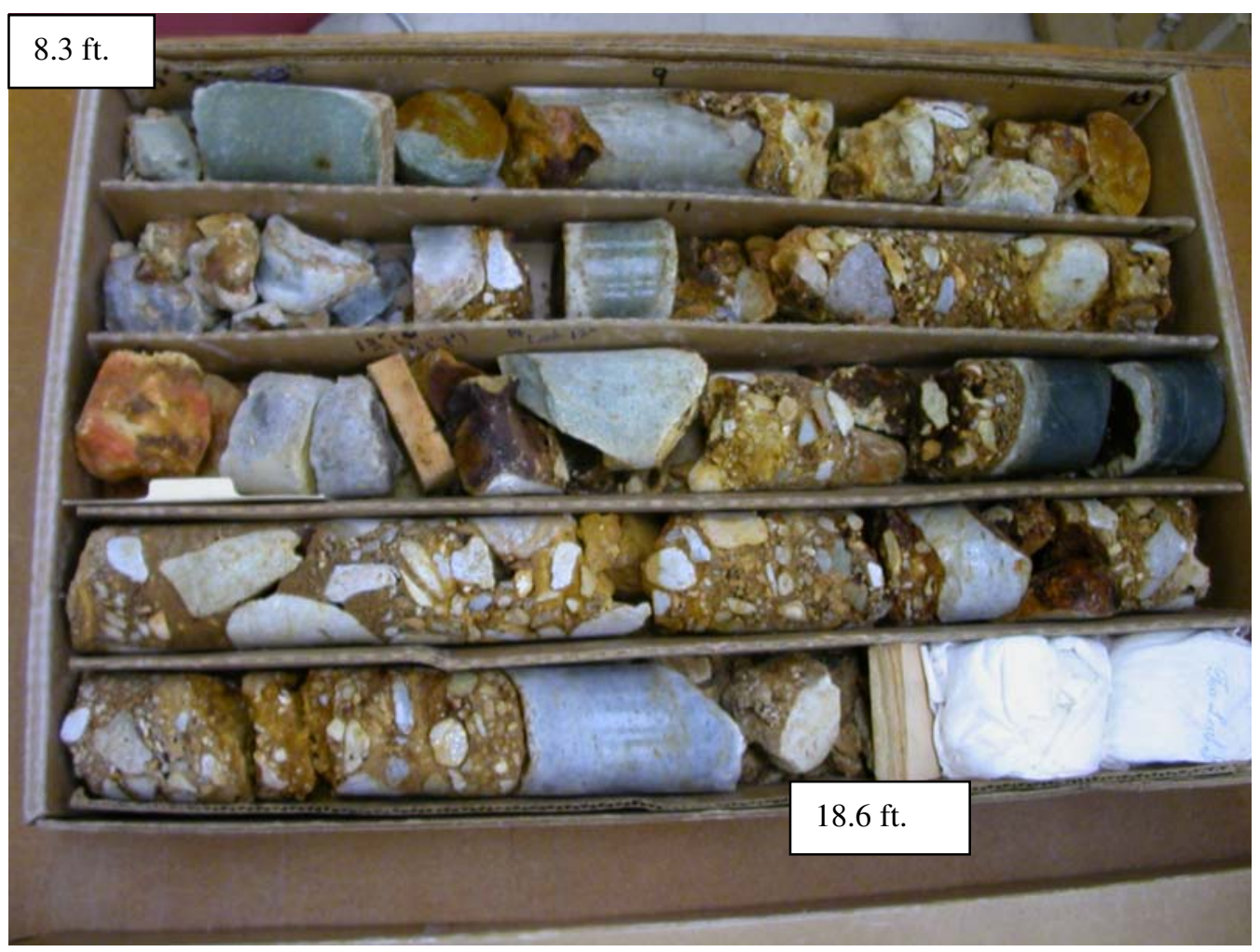




\section{Interval from 18.6 to $35 \mathrm{ft}$.}

Alluvial ferricrete as above. Consists of cobbles and subrounded to angular pebbles in an iron-oxide cemented matrix. Reverse grading in pebble layers between subrounded cobbles. Most clasts are propylitic-altered Tsv lavas with some larger quartz-alunite and quartz-pyrophyllite-altered clasts (latter two alteration lithologies from about 28-30 ft.).

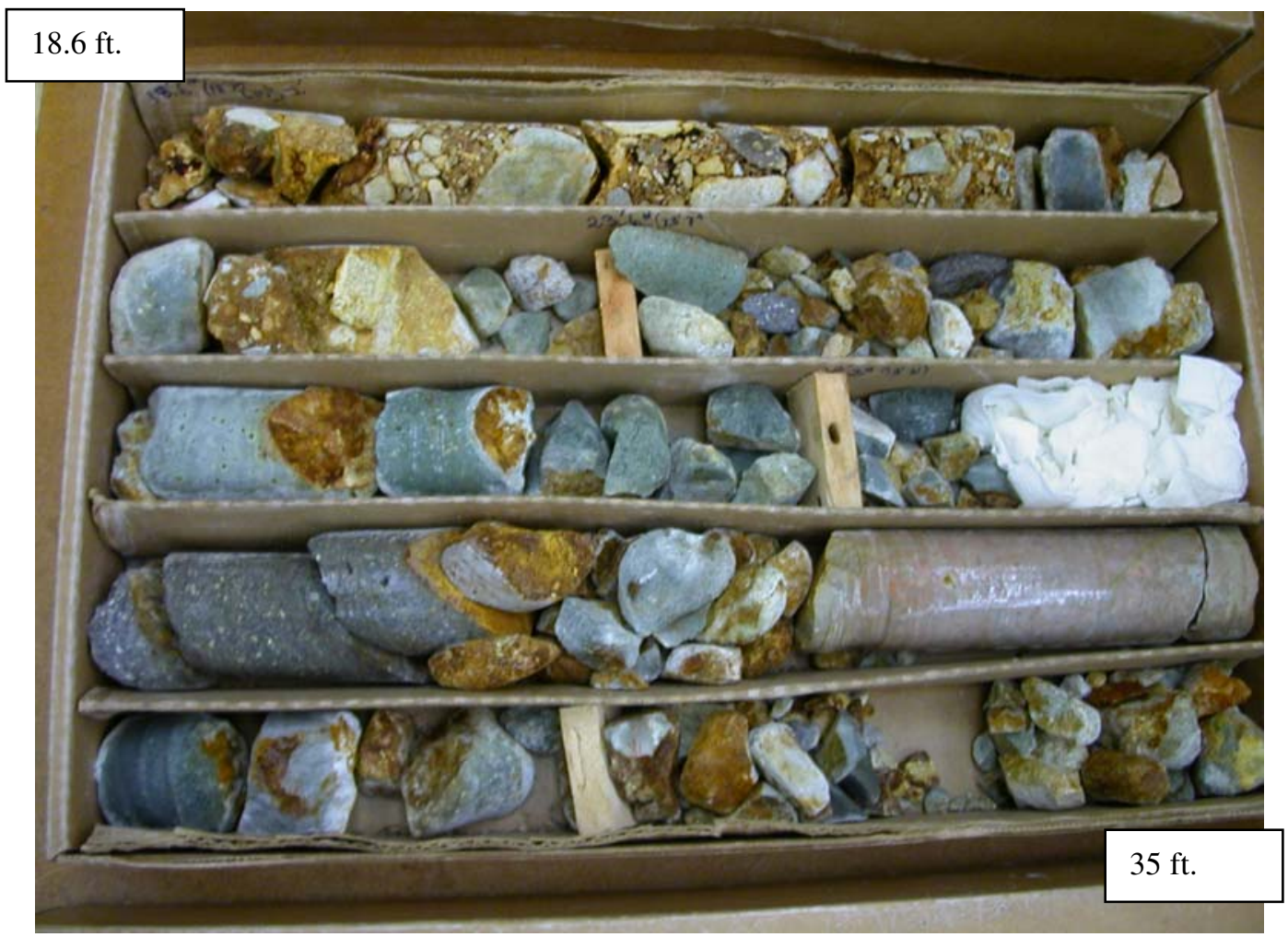




\section{Interval from 35 to $73.7 \mathrm{ft}$.}

Glacial till? Angular to subrounded pebble to cobble-sized clasts in a gray brown, clay-rich matrix cement. Fine-grained $(<1 \mathrm{~mm})$ pyrite in clayey matrix is mostly unoxidized; matrix is moderately indurated. Clasts are largely propylitic to weak sericitic altered Tsv lavas.
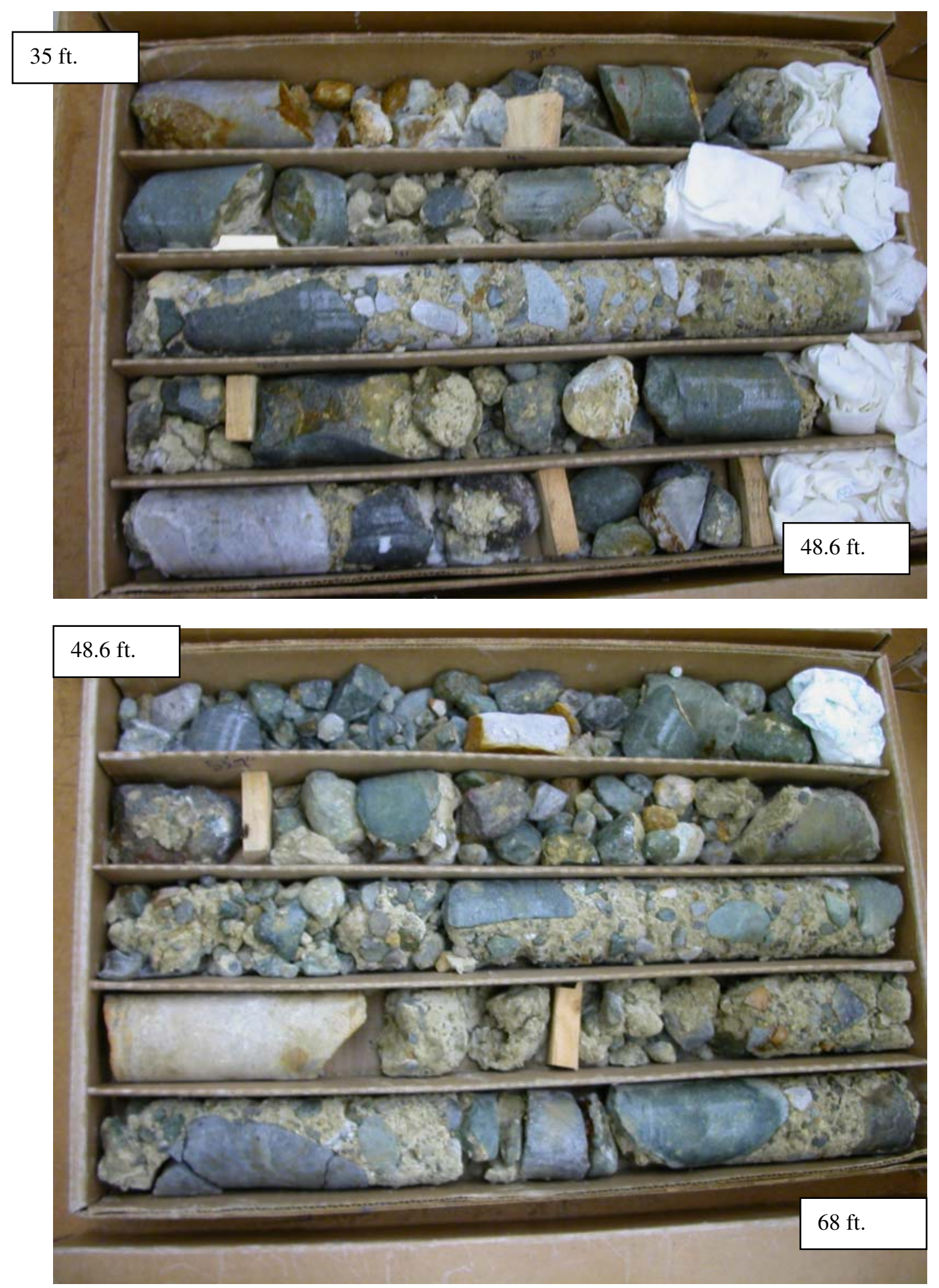


\section{Interval from 73.7 to $88.7 \mathrm{ft}$.}

Saprolitic top of Tsv lava bedrock. Top several feet of core is rubbly and clay altered, with abundant light brown-yellow clays (sericite and kaolinite). Rock below saprolite is highly fractured and broken Tsv; alteration ranges from propylitic to weak sericitic with jarosite and goethite that replaced pyrite along fractures. Fine-grained pyrite is sparse in groundmass. Quartz vein at about $85 \mathrm{ft}$; vein is $1 \mathrm{~cm}$ wide with cockscomb texture and is iron-stained. Similar quartz vein is present at bottom of this interval; vein is associated with clay (possibly sericite).

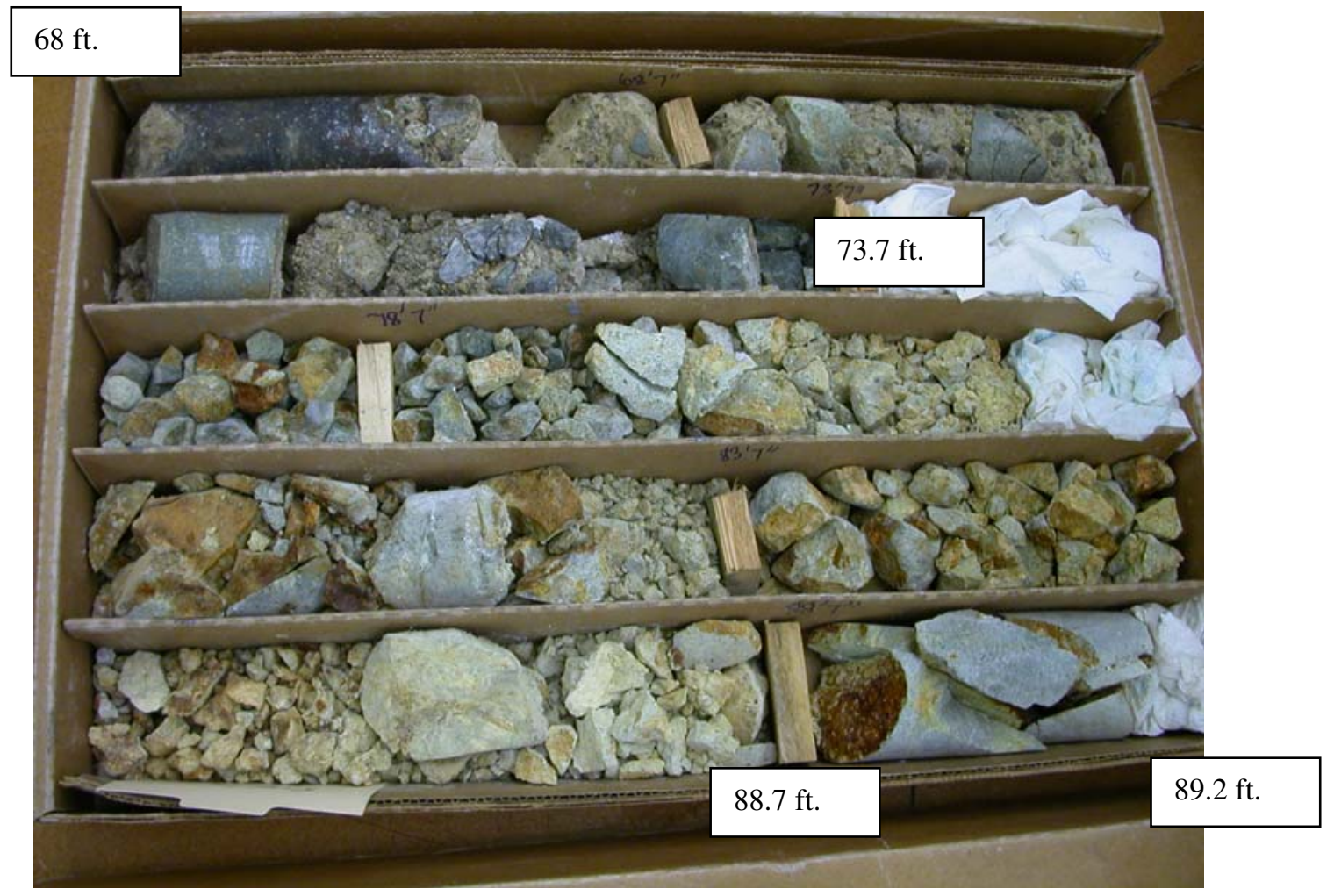




\section{Interval from 88.7 to $108 \mathrm{ft}$.}

Highly fractured, propylitic to weak sericitic-altered Tsv lavas. Secondary albite is abundant in these rocks (10-30\%). Contains plagioclase phenocrysts $(<1-2 \mathrm{~mm})$ replaced by chlorite and epidote. Interval cut by moderately dense network of 1-2 mm quartz-pyrite veinlets; late white kaolinite is present along fracture surfaces. Goethite and hematite also are present along some fractures faces. Fine-grained pyrite is present mostly along fractures but is sparse $(<1 \%)$ in the groundmass; overall content is $1-3 \%$. Tsv lavas become a bit more coarsely porphyritic at $107-108 \mathrm{ft}$. with plagioclase up to $2 \mathrm{~cm}$; phenocrysts aligned along foliation.
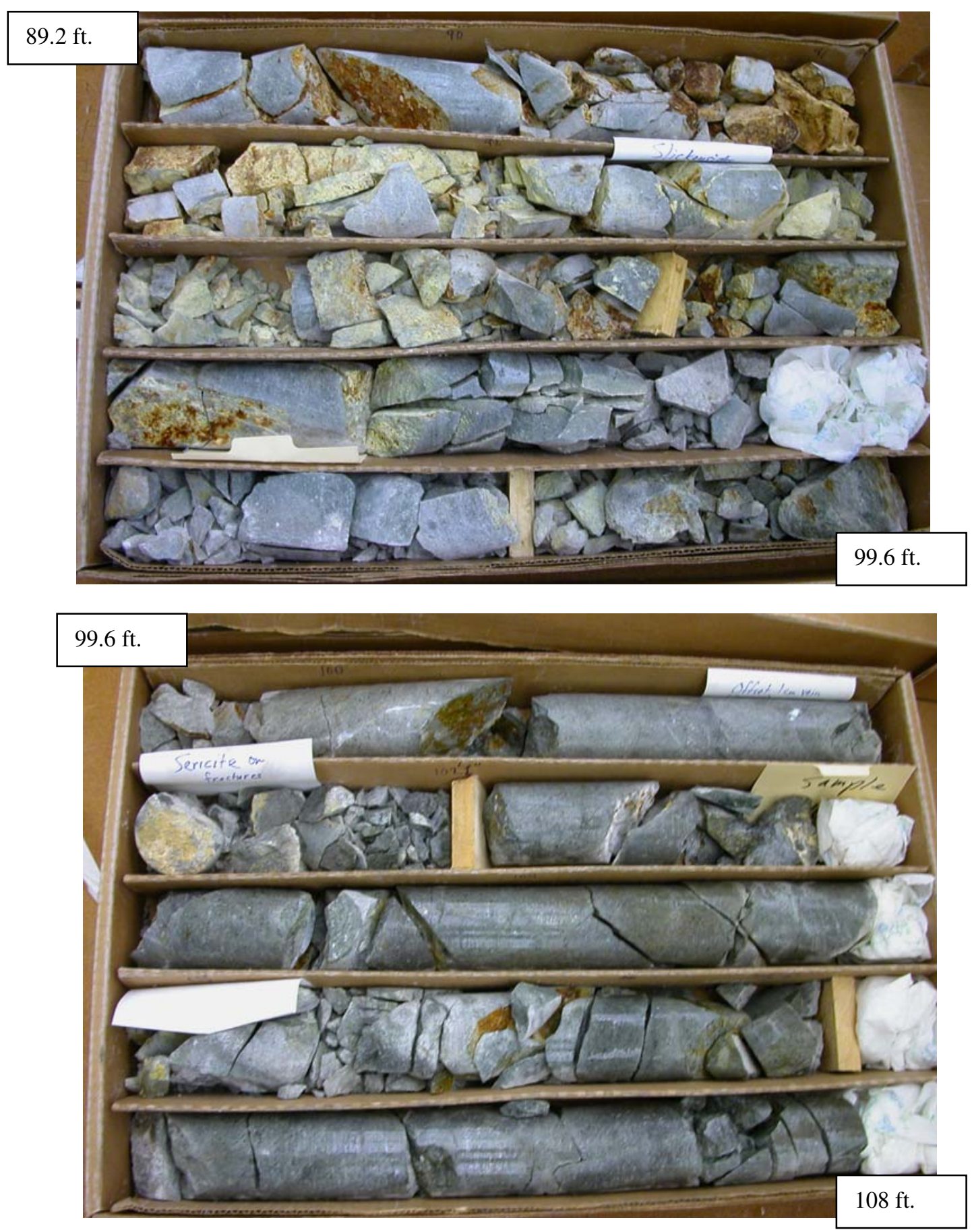


\section{Interval from 108 to $117 \mathrm{ft}$.}

Propylitic-altered Tsv lavas with strong secondary albitization ( $>35 \%$ albite); chlorite is abundant and minor epidote is present locally. Rock is intensely fractured in a few intervals; pyrite $(<1 \mathrm{~mm})$ is present on fracture surfaces and in some narrow quartz-pyrite veinlets, some also with sericite. Pyrite content is $<1 \%$.

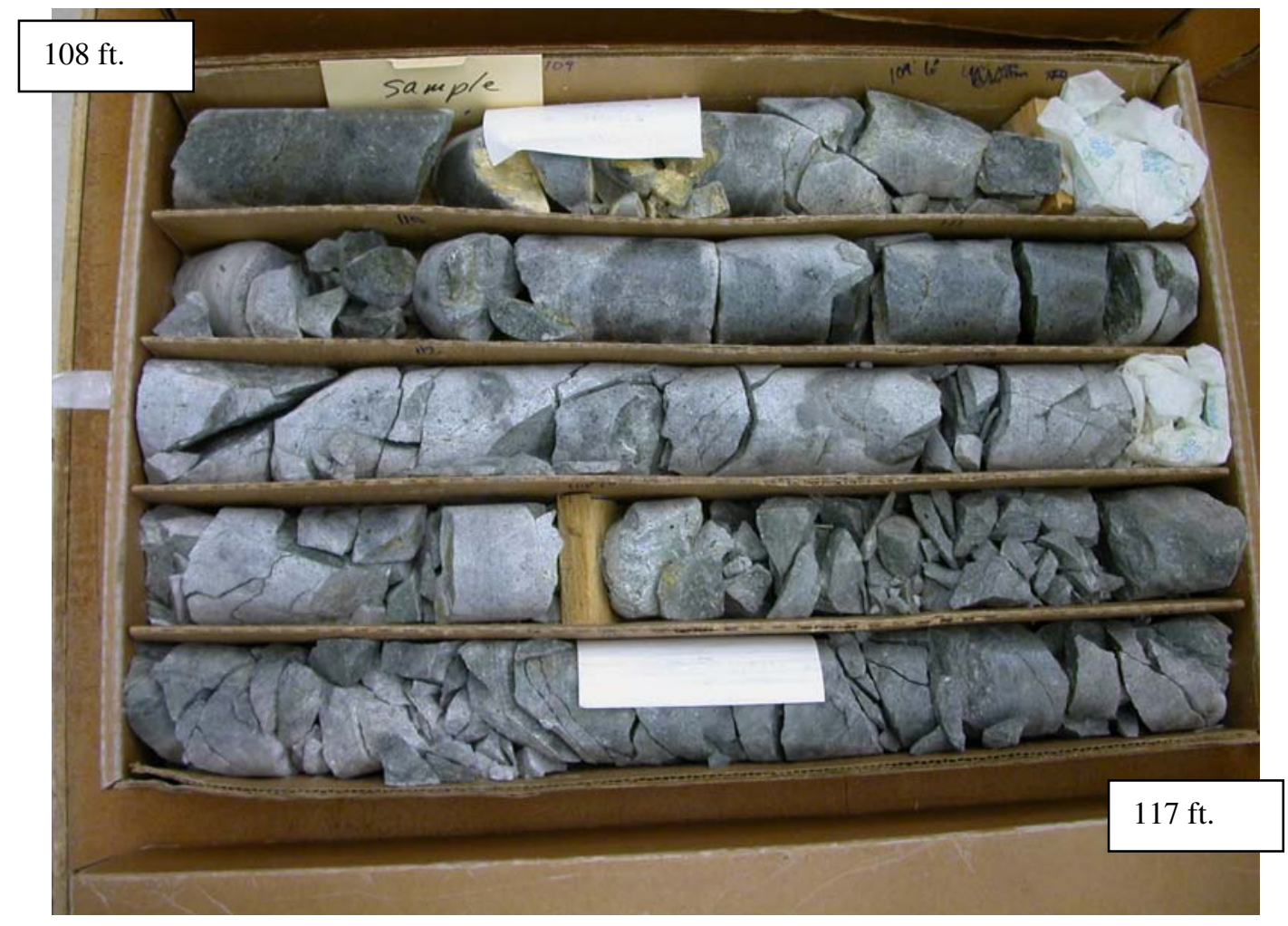




\section{Interval from 117 to $127 \mathrm{ft}$.}

Entire interval is highly fractured and broken; weak sericitic to Qsp-altered Tsv lavas. Plagioclase (only about $5 \%$ albite remains) is mostly altered to sericite and minor chlorite (epidote appears to be partially to wholly replaced). Mafics to sericite but some chlorite remains. Rocks are weak to moderately silicified. Irregular and thin pyrite veinlets are sporadic, some with quartz. Pyrite abundance is $1-2 \%$. This altered zone marks the upper alteration halo of hydrothermal alteration/ veining centered around a strong fracture zone at 140-142 ft. (see description below).

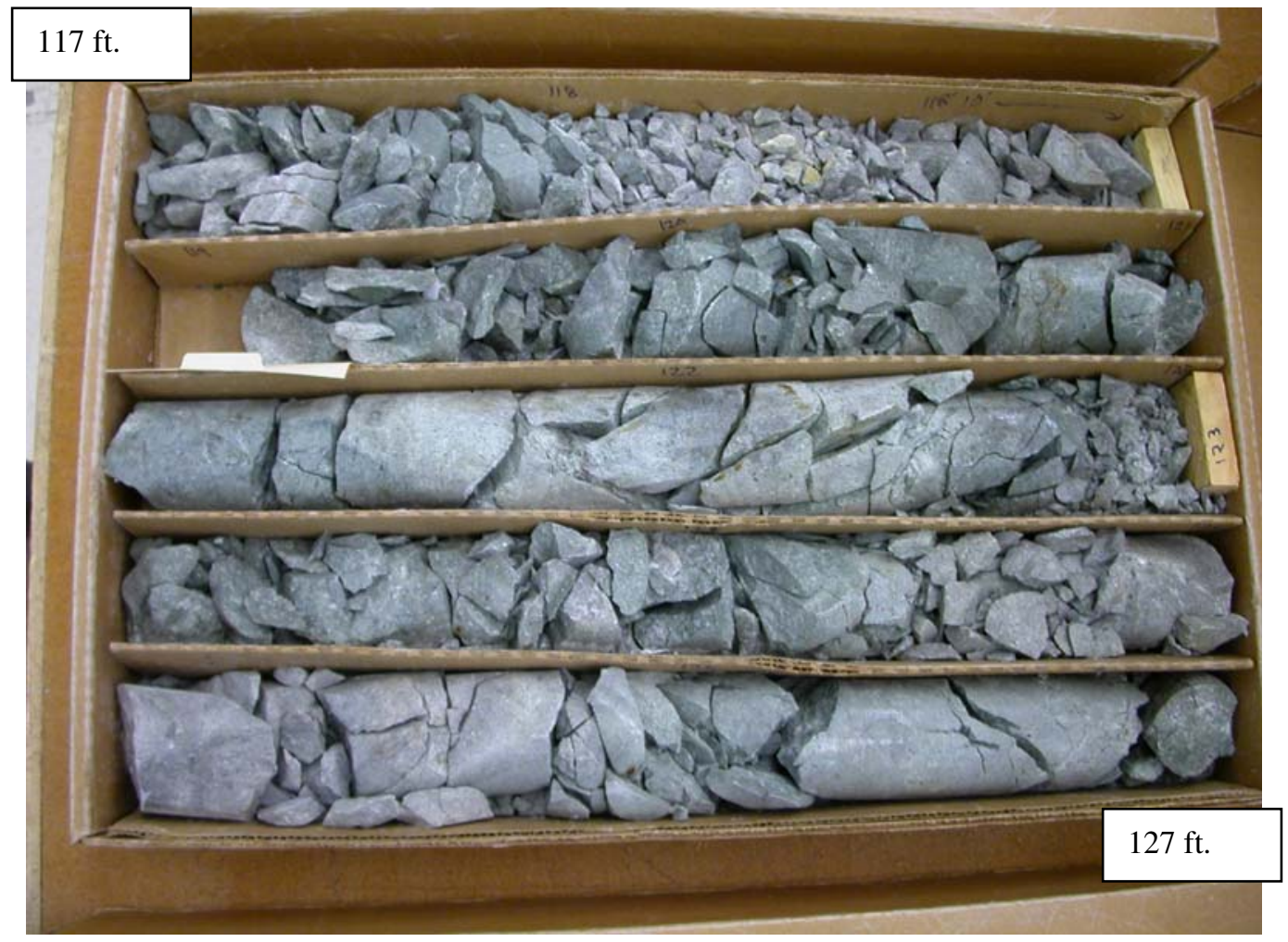




\section{Interval from 127 to $135.3 \mathrm{ft}$.}

Matrix-supported breccias; Qsp and moderately silicified; contain abundant subangular clasts that are rather evenly distributed. Clasts tend to be fairly even in size, averaging about $0.5 \mathrm{~cm}$; clasts were derived from a dark gray, fine or chalcedonic quartz-altered rock; the dark gray clasts comprise $15-20 \%$ of the brecciated rock. Rocks cut by local dark gray, chalcedonic quartz flooded zones (up to $1 \mathrm{~cm}$ ) that were localized along angular fractures. Note the near vertical, dark, chalcedonic quartz-flooded fracture $(2-3 \mathrm{~cm})$ at $134 \mathrm{ft}$.; this zone has very diffuse margins. This feature continues to $139 \mathrm{ft}$. in the next core interval. Fine-grained pyrite abundance is $4-5 \%$.

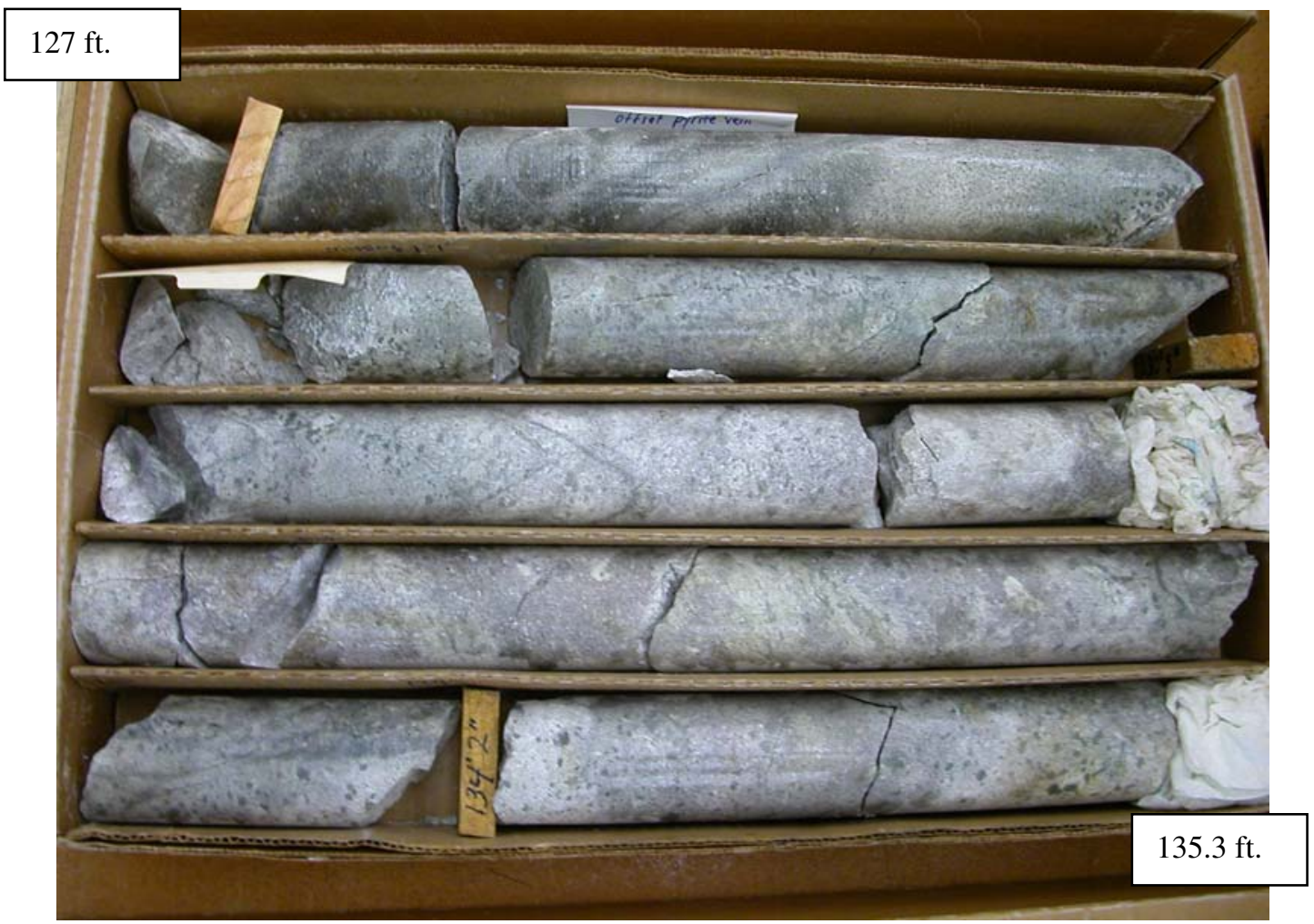




\section{Interval from 135.3 to $144 \mathrm{ft}$.}

Matrix-supported breccia with dark, evenly distributed clasts continues to $142 \mathrm{ft}$. These breccias are weak sericite-pyrite altered, contain partially altered k-spar and secondary albite, are moderately silicified, and have metastable chlorite. The near vertical chalcedonic quartz vein tapers out at $138 \mathrm{ft}$. Matrix-supported breccias appear to grade into a coarse-grained, clast-supported breccia in the interval from 139 to $142 \mathrm{ft}$. The texture in the 139-142 ft. interval is obscured by strong overprint of medium to dark gray, chalcedonic silicification (> 52\% quartz; feldspars completely altered to sericite; chlorite still present along fractures). A near vertical, $0.5 \mathrm{~cm}$ fracture is filled by milky open-space quartz (vein composed of anhedral crystals). The white quartz vein cuts both the coarse breccia interval and finer matrix-supported breccia (143-144 ft.). The matrixsupported breccia at $143-144 \mathrm{ft}$. appears to be a gradational transition from the coarser breccia. In summary: hydrothermal? breccia is localized in the interval from $127-151.5 \mathrm{ft}$. A coarse breccia is localized at the core of this zone and grades into finer breccias on both margins. Chalcedonic quartz is localized along fractures and post-dates brecciation. The coarse breccia interval from 139-142 ft. has localized dark chalcedonic quartz flooding; this may be equivalent to that seen along strong vertical fracture from 139-142 ft. Episodes of brecciation and chalcedonic silicification are post-dated by fracture-filled quartz vein. The white quartz vein is offset $\sim 0.5 \mathrm{~cm}$ by a small normal fault at $141 \mathrm{ft}$. Hydrothermal alteration and quartz veining appears to have been localized near the coarse core of the breccia zone that extends from 127 to $151 \mathrm{ft}$. Qsp alteration and silicification extends about $12 \mathrm{ft}$. on either side of this zone. Question: what/where is the source of the evenly distributed breccia clasts? Fine-grained pyrite content is $5-6 \%$ to about $139 \mathrm{ft}$. and diminishes to $<2 \%$ from 139 to $144 \mathrm{ft}$.

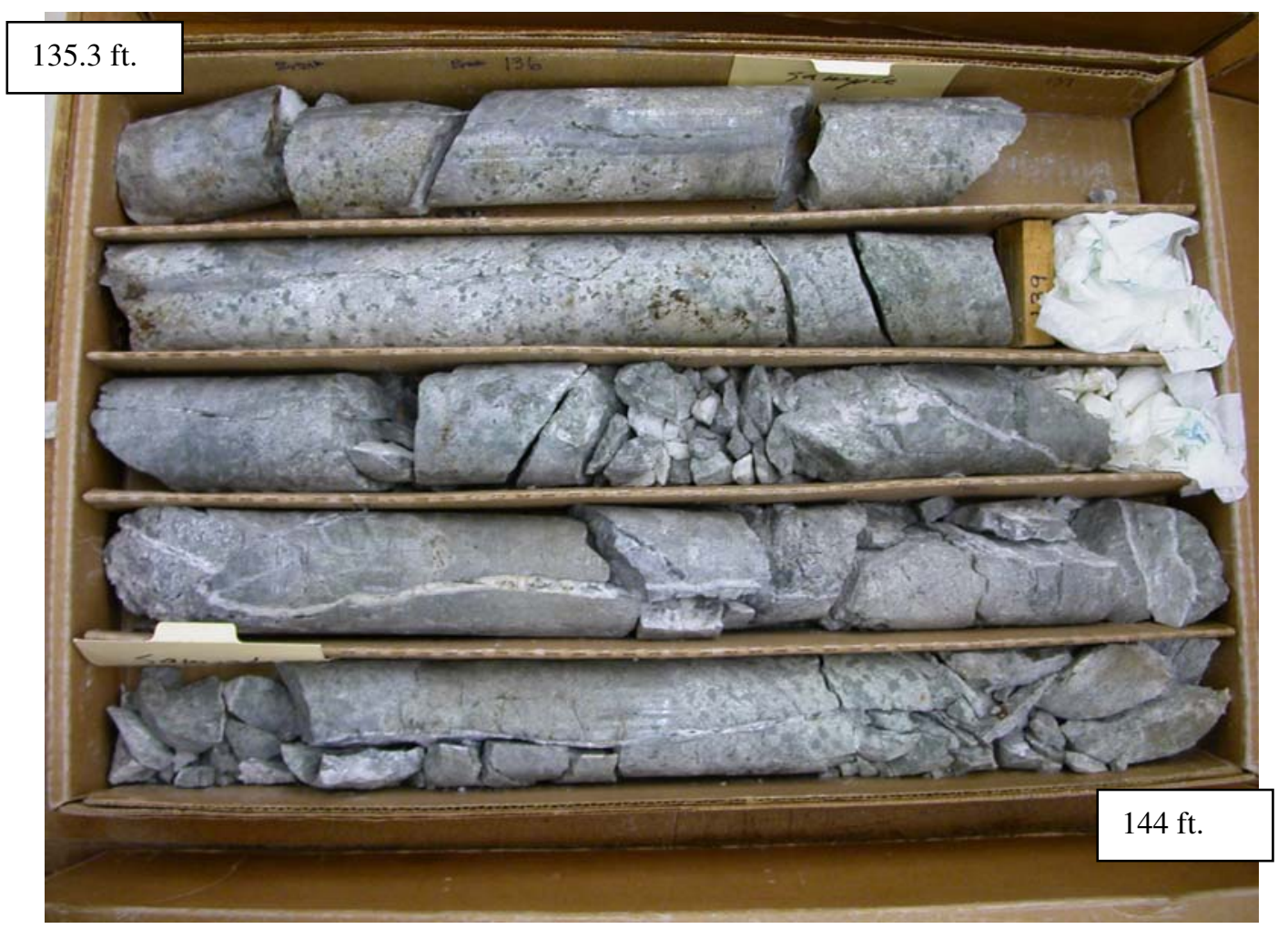




\section{Interval from 144 to $151 \mathrm{ft}$.}

Qsp to weak sericitic-altered, moderately silicified breccias (weak sericite-pyrite altered, contain partially altered k-spar, secondary albite, and chlorite along fractures); $1 \mathrm{~mm}$ pyrite (as fracture-fillings and in sporadic quartz veins) $<1-2 \%$. Breccias still contain $0.5-1 \mathrm{~cm}$ silicified clasts. Fracture with slickensides at $151 \mathrm{ft}$.

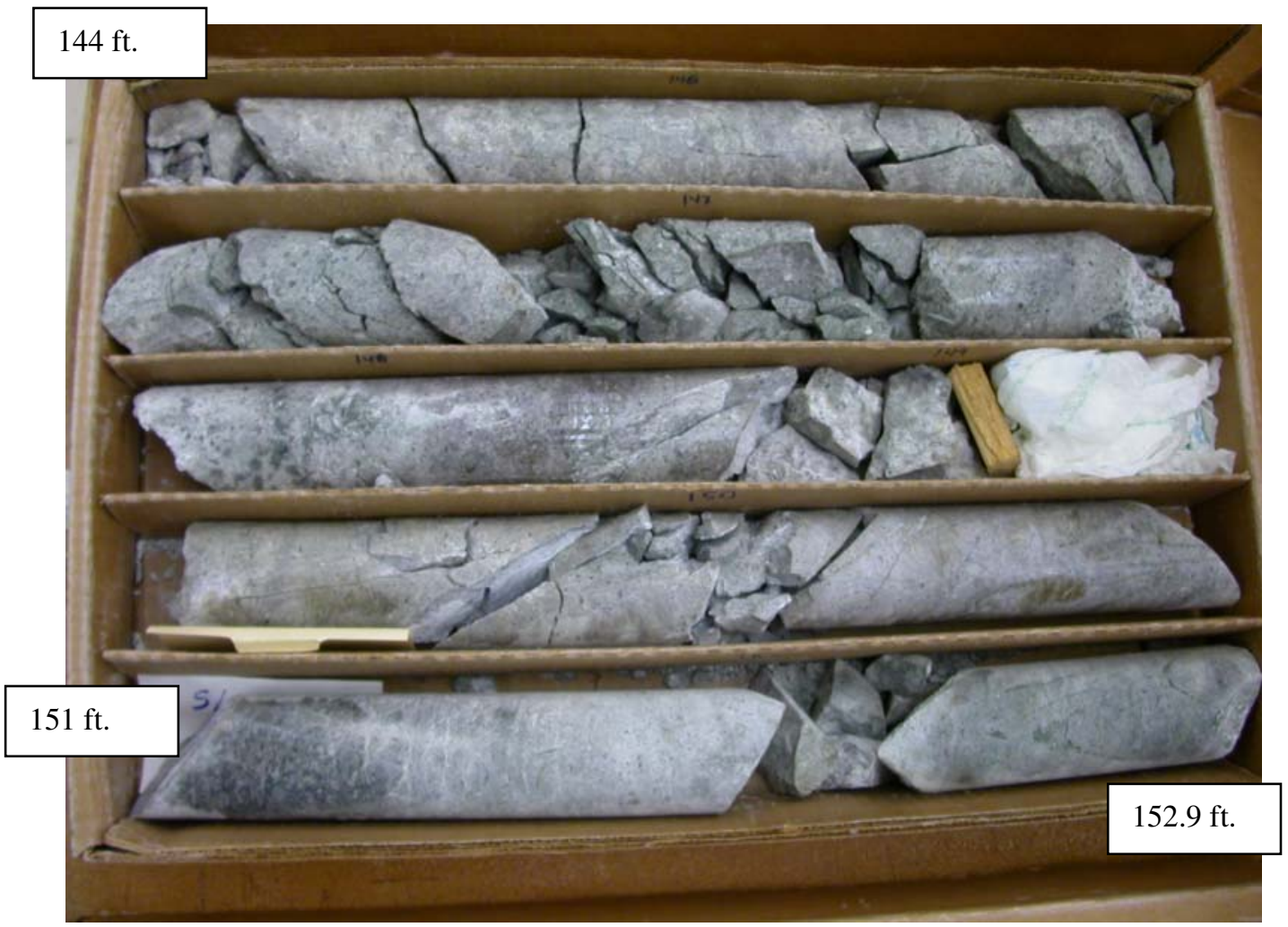




\section{Interval from 151 to $159 \mathrm{ft}$.}

Breccias transition into weak sericitic to propylitic-altered, flow-foliated lavas (Tsv) at $151.5 \mathrm{ft}$; lavas contain $1 \mathrm{~mm}$ wide phenocrysts. Rocks are strongly albitized. Albite and k-spar are weak to moderately sericitized. Biotite is metastable and along with other mafics is partially altered to chlorite and epidote. Moderately dense fracture network is filled with dark chalcedonic quartz and pyrite (not quite stockwork texture). Late, white kaolinite is also present along many of these fractures. Tsv lavas are cut by mediumgrained, clast-supported hydrothermal breccia at $159 \mathrm{ft}$. Breccia has very sharp margins. Fine pyrite $<1-2 \%$.

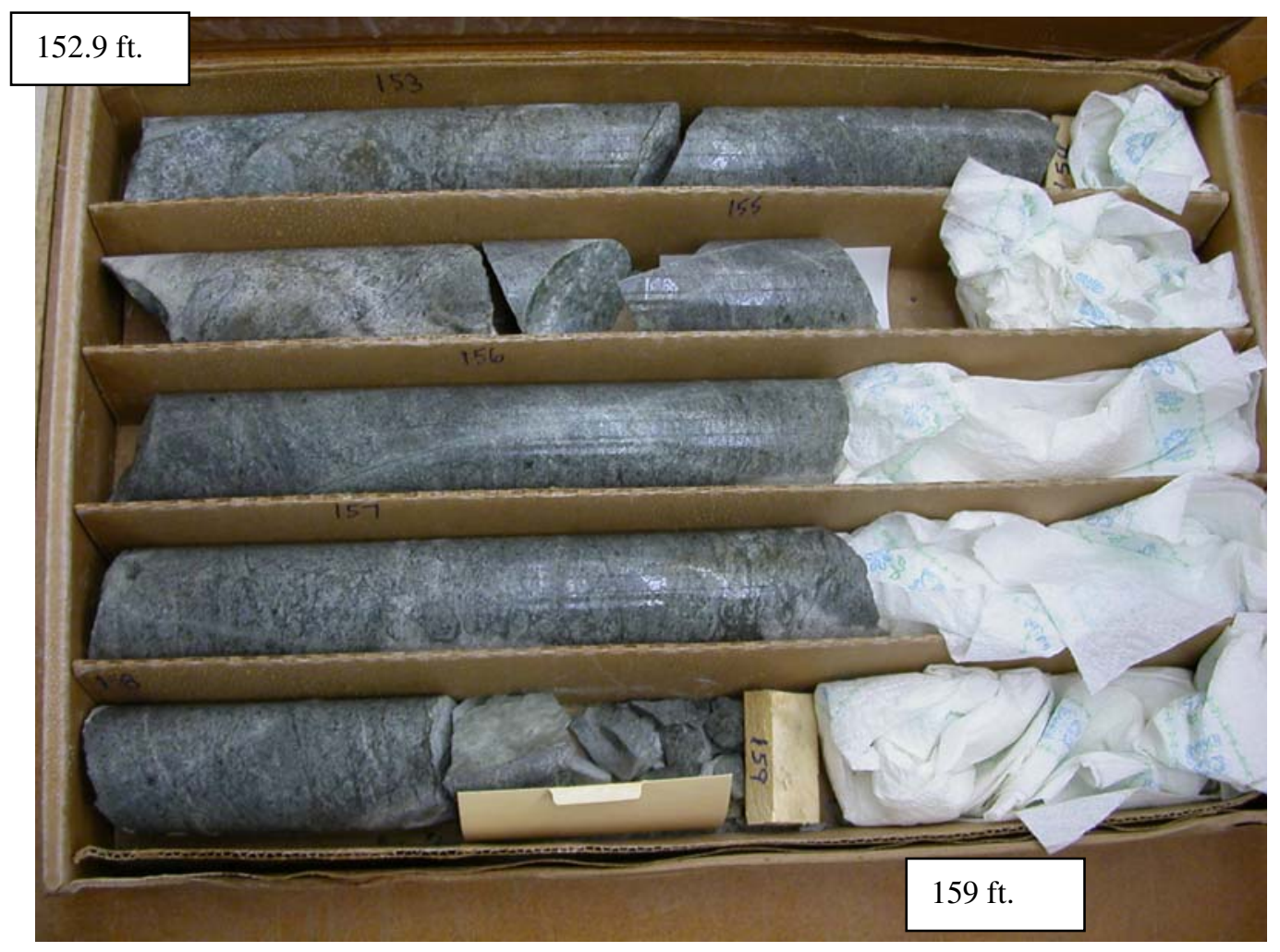

